UNIVERSIDAD AUTÓNOMA METROPOLITANA UNIDAD IZTAPALAPA

DIVISIÓN DE CIENCIAS BÁSICAS E INGENIERÍA

"Detección del flip-flop de los centros paramagnéticos del GaAsN mediante bombeo óptico en régimen pulsado".

Tesis que presenta:

M. en C. Juan Carlos Sandoval Santana.

Para obtener el grado de

Doctor en Ciencias (Física).

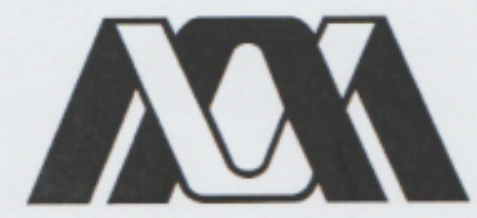

Casa abierta al tiempo

Asesor: Dr. Alejandro Kunold Bello

Jurado:

Presidente: Dr. Pedro Pereyra Padilla

Secretario: Dr. Alejandro Kunold Bello

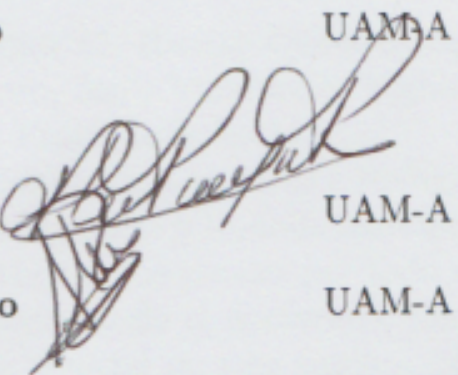

Vocal: Dr. Pablo de la Mora y Palomar Askinasy FC-UNAM

$1.04 \mathrm{H} / \mathrm{h}$

Vocal: Dr. Gerardo García Naumis

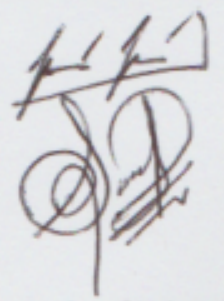

IF-UNAM

Vocal: Dr. Jaime Santoyo Salazar

CINVESTAV-IPN

Ciudad de México

6 de diciembre de 2018 


\section{ACTA DE DISERTACIÓN PÚBLICA}

Detección del flip-flop en los centros paramagneticos del GaAsN mediante bombeo optico en régimen pulsado.

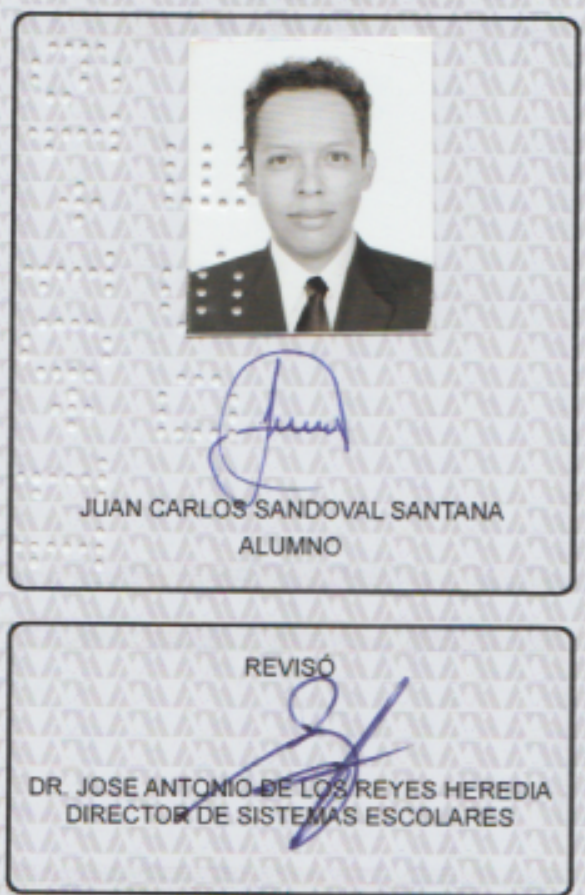

DIRECTOR DE LA DIVISIÓN DE CBI

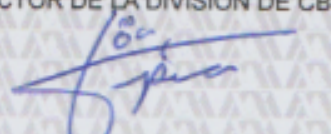

DR. JESUS ALEERTO OCHOA TAPIA
En la Ciudad de México, se presentaron a las 17:00 horas del dí 13 del mes de diciembre del año 2018 en la Unidad Iztapalapa de la Universidad Autonoma Metropolitana, los suscritos miembros del jurado:

DR. PEDRO PEREYRA PADILLA

DR. GERARDO GARCIA NAUMIS

DR. PABLO DE LA MORA Y PALOMAR ASKINASY

DR. JAIME SANTOYO SALAZAR

DR. ALEJANDRO KUNOLD BELLO
Bajo la presidencia del primero y con caracter de Secretario el último, se reunieron a la presentación de la Disertacion Pública cuya denominacion aparece al margen, para la obtención del grado de:

DOCTOR EN CIENCIAS (FISICA)

DE: JUAN CARLOS SANDOVAL SANTANA

y de acuerdo con el articulo 78 fraccion IV del Reglamento de Estudios Superiores de la Universidad Autonoma Metropolitana, los miembros del jurado resolvieron:

\section{- aprobar}

Acto continuo, el presidente del jurado comunicó al interesado el resultado de la evaluacion $y$, en caso aprobatorio, le fue tomada la protesta.

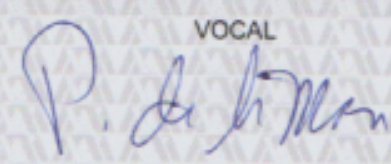

DR. PABLO DE LAMORA Y PALOMAR ASKINASY
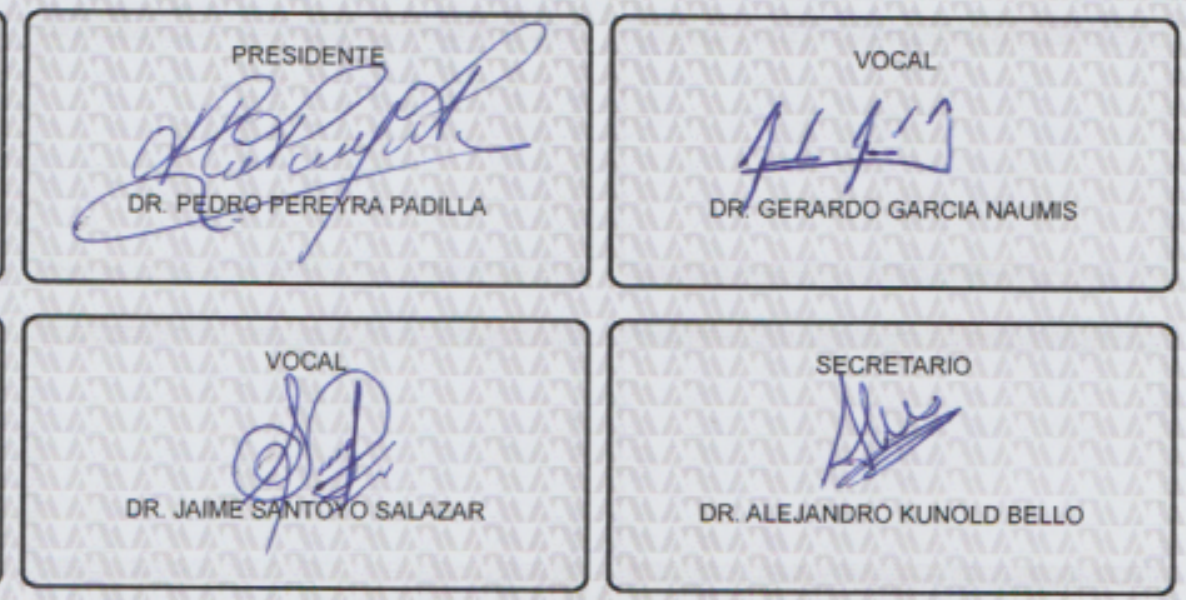

DR. ALEJANDRO KUNOLD BELLO 
UNIVERSIDAD AUTÓNOMA METROPOLITANA

UNIDAD IZTAPALAPA

DIVISIÓN DE CIENCIAS BÁSICAS E INGENIERÍA

"Detección del flip-flop de los centros paramagnéticos

del GaAsN mediante bombeo óptico en régimen pulsado".

Tesis que presenta:

M. en C. Juan Carlos Sandoval Santana.

Para obtener el grado de

Doctor en Ciencias (Física).

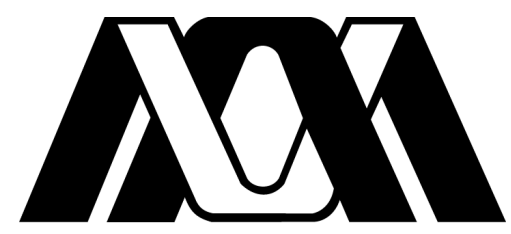

Casa abierta al tiempo

Asesor: Dr. Alejandro Kunold Bello

UAM-A

Jurado:

Presidente: $\quad$ Dr. Pedro Pereyra Padilla

UAM-A

Secretario: Dr. Alejandro Kunold Bello

UAM-A

Vocal: Dr. Pablo de la Mora y Palomar Askinasy FC-UNAM

Vocal: Dr. Gerardo García Naumis

IF-UNAM

Vocal: Dr. Jaime Santoyo Salazar

CINVESTAV-IPN

Ciudad de México

6 de diciembre de 2018 
A mi esposa Gaby y mi hija Eleni Renée... "La felicidad requiere algo que hacer, algo que amar y algo que esperar". 


\section{Agradecimientos}

Por haberme dado la oportunidad de ser parte de su equipo de trabajo, agradezco al Dr. Alejandro Kunold Bello, Dr. José Luis Cardoso Cortés y Dr. Victor G. Ibarra Sierra. "Las huellas que caminaron juntas nunca se borran".

Gracias al Dr. Pedro Pereyra Padilla, al Dr. Gerardo García Naumis, al Dr. Pablo de la Mora y Palomar Askinasy y al Dr. Jaime Santoyo Salazar por los comentarios hechos a este trabajo los cuales ayudaron a mejorar bastante este trabajo.

A los profesores Andréa Balocchi y Thierry Amand de la Universite de Toulouse, INSA-CNRS-UPS, LPCNO (Francia) y a los profesores Vlamidir Kalevich, Eugeneous Ivchenko y Leonid Bakaleinikov del Ioffe PhysicalTechnical Institute (Rusia), por la colaboración realizada en el tema central de esta tesis.

A la M. en C. María Iseo González Christen por su enorme paciencia, eficiencia y apoyo en los diferentes tramites académicos.

A la Universidad Autónoma Metropolitana, por el apoyo económico otorgado para realizar los estudios de doctorado.

Por su cariño y amistad les agradezco Victor G. Ibarra, Gustavo Mejía, Aarón García, Julio Hidalgo, Martín Romero, Jairo Villafuerte, Yoshua Bolaños a otros tantos amigos del posgrado en Física de la UAM-I.

Por el apoyo, confianza y amor que me han brindado, agradezco a Refugio Sandoval, Leonida Santana, Marco Antonio, Freddy Jahir y Rodolfo Santana.

Le agradezco inmensamente a mi esposa Gabriela, gracias por todo el apoyo, comprensión y amor que me brindo duratne todo el doctorado. Este logro es trabajo en equipo, mi esposa es parte esencial de este equipo. "El corazón llega donde no llegan los pies". 


\section{Resumen}

U

sando el enfoque de la ecuación maestra, en este trabajo desarrollamos un modelo que describe la dinámica del espín nuclear y electrónico en régimen pulsado en trampas paramagnéticas de $\mathrm{Ga}^{2+}$ presentes en semiconductores de GaAsN. Este modelo describe las principales interacciones entre los electrones de la banda de conducción, los huecos de la banda de valencia y los centros paramagnéticos. Estas interacciones son el mecanismo de la recombinación dependiente del espín, la interacción hiperfina en las trampas paramagnéticas, las interacciones de Zeeman en los electrones de la banda de conducción y de las trampas. Con los resultados del modelo, proponemos un esquema de pulso-prueba óptico que nos permite trazar y detectar el flip-flop del espín electrón-núcleo de los centros en el GaAsN en evolución temporal [1]. Las predicciones del modelo fueron confirmadas experimentalmente [2] dando resultados notablemente parecidos a los predichos teóricamente. Los resultados experimentales muestran que los procesos de filtrado y de polarización del espín tienen fluctuaciones debido al flip-flop causado por la interacción hiperfina en las trampas. Las fluctuaciones en el filtrado fueron vistas a través de la intensidad de la fotoluminiscencia y la razón de recombinación dependiente del espín. Además, mostramos cuáles son las condiciones óptimas del experimento para detectar el flip-flop en las tres direcciones espaciales [3]. Este esquema es totalmente óptico y no requiere campos magnético externos y/o radiaciones de microondas como los métodos de resonancia magnética nuclear. 


\section{Índice general}

Resumen VII

$\begin{array}{ll}\text { Índice de figuras } & 1\end{array}$

1. Introducción 3

2. Marco teórico 9

2.1. Trampas paramagnéticas en GaAsN . . . . . . . . . . . . 9 9

2.2. Enfoque de la ecuación maestra . . . . . . . . . . . . . 12

2.2.1. Álgebras de Lie . . . . . . . . . . . . . . . . . . . 13

2.2 .2 . Inyección de espín en GaAsN $\ldots \ldots \ldots \ldots$

2.2 .3$. Modelo de dos cargas . . . . . . . . . . . . 16

3. Modelo de la dinámica del espín en GaAsN 19

3.1. Matriz de densidad . . . . . . . . . . . . . . . . . . . . 19

3.2. Espacio prehilbertiano . . . . . . . . . . . . . . 21

3.3. Hamiltoniand . . . . . . . . . . . . . . . . . . . 24

3.4. Disipador . . . . . . . . . . . . . . . . . . 24

4. Resultados 29

4.1. Resultados para un isótopo . . . . . . . . . . . . . . . . . 32

4.2. Resultados experimentales y teóricos para dos isótopos . . . . 38

4.3. Régimen pulsado en presencia de un campo magnético oblicuo a la dirección de polarización $\ldots \ldots \ldots \ldots$. . . . . . . . . . 42

4.3.1. Optimización de la potencia . . . . . . . . . . . . . 44

4.3.2. Optimización del campo magnético . . . . . . . . . . . 47

5. Conclusiones y perspectivas 53

\begin{tabular}{ll}
\hline A. Operadores & 57
\end{tabular}

A.1. Operadores del hamiltoniano . . . . . . . . . . . . . . . 57 
B. Ecuación maestra para dos isótopos de $\mathrm{Ga}^{2+} \quad 59$

B.1. Modelo para dos isótopos de $\mathrm{Ga}^{2+}$. . . . . . . . . . . . . 59

\begin{tabular}{ll}
\hline C. Artículos publicados & 65
\end{tabular} 


\section{Índice de figuras}

2.1. El mecanismo de la SDR consiste en cuatro pasos: Primero, excitamos electrones de la $\mid$ BV|a la $\mid$ BC la polarización neta de espín es diferente de cero $(S \neq 0)$ en la $|\mathrm{BC}| 2.1$ (a). Segundo, los fotoelectrones en la $|\mathrm{BC}|$ caen en los centros paramagnéticos, siempre y cuando el espín de los electrones de la|BC|sea opuesta al espín electrónico de las trampas, formando, junto con los electrones de las trampas, pares de electrones 2.1 (b). Tercero, uno de los dos electrones apareados en las trampas cae en un hueco de la BV 2.1 (c). La primera parte del proceso de recombinación se lleva acabo en un tiempo mucho menor que la segunda; los centros son saturados después se vacían y se polarizan en la misma dirección que los electrones de la BC $2.1(\mathrm{~d})] \ldots \ldots \ldots \ldots 11$

2.2. Diagrama de las reglas de selección entre la BC y la BV. Las transiciones inducidas por luz circularmente polarizada hacia la izquierda están de color azul mientras que las transiciones debio a la luz circularmente polarizada hacia la derecha están de color verde. La probabilidad de transición de un estado de la BV a la BC o también llamada fuerza de oscilador se indica con un número al lado de cada transición . . . . . . 15

2.3. Esquema del flujo de la carga entre la banda de conducción, las trampas paramagnéticas y la banda de valencia. La carga fluye desde la banda de conduccón, transita por las trampas paramagnéticas y termina en la banda de valencia. Este flujo de carga está descrito por un conjunto de ecuaciones fenomenológicas denominado modelo de dos cargas. [4, 5] . . . 16

3.1. La placa superior gris representa la BC donde se encuentran los electrones polarizados. La placa inferior representa la BV donde se encuentran los huecos. Entre las placas están las trampas paramagnéticas apareadas que tienen dos electrones y las no apareadas que tienen un electrón. . . . . . 20 
4.1. En la Figura (a) mostramos la $S D R_{r}$ en función del tiempo en ausencia de campo magnético. Notamos que el $S D R_{r}$ tiene un máximo al pulso lanzado. En la Figura (b), presentamos las polarizaciones de espín electrónica de la BC ( $S_{z}$ curva roja), de espín electrónica de las trampas no apareadas ( $S_{c z}$ curva café), de espín nuclear de las trampas no apareadas $I_{1 z}$ (curva verde) y de espín nuclear de las trampas apareadas $I_{2 z}$ (curva azul) en ausencia de un campo magnético . Las polarizaciones $I_{2 z}$ y $S_{z}$ crecen hasta un tiempo máximo y depués decrecen monotonamente hasta cero. Las curvas $I_{1 z}$ y $S_{c z}$ tienen un comportamiento sinosoidal evanescente. El comportamiento oscilante se le denomina flip-flop el cual es debido a la interacción hiperfina entre los espines nuclear y electrónico de la trampa no apareada. El comportamiento evanescente se debe a las perdidas de espín electrónico y nuclear . . . . . . . . . . . . . . . . 32

4.2. En la Figura (a) mostramos la $S D R_{r}$ en función del tiempo en ausencia de un campo magnético externo en presencia de dos pulsos consecutivos. Notamos que el $S D R_{r}$ tiene dos máximos, esto se debe a la presencia de los dos pulsos. Observamos que el máximo del segundo pulso es más grande que el primero. Este aumento en el $S D R_{r}$ se debe a la información remanente del primer pulso. En la Figura (b), mostramos las curvas de polarización electrónica $\left(S_{c z}\right.$ curva azul) y nuclear ( $I_{1 z}$ curva naranja) de las trampas no apareadas. Las curvas de polarización muestran el flip-flop de espín electrón-núcleo causado por la interacción hiperfina en las trampas no apareadas. Dichas curvas tienen un cambio abrupto en $t=270$ ps debido a la incidencia del segundo pulso. Notamos que este aumento en las polarizaciones Figura (b) se ve reflejado en el aumento del segundo pico . . . . . . . . . . . . . . . . . . . . . . . 33

4.3. Bosquejo de la propuesta del experimento. La propuesta del experimento se centra en realizar varias pruebas donde en cada prueba incidimos dos pulsos consecutivos con la misma intensidad en ausencia de un campo magnético externo para los casos de luz circularmente polarizada y luz linealmente polarizada con el fin de medir $S D R_{r}=I_{-} / I_{x}$ en cada prueba. Para cada situación se mantiene fijo el tiempo de incidencia del primer pulso $\left(t_{10}\right)$ y se aumenta el tiempo de incidencia del segundo pulso en una proporición de $t_{2}=t_{20}+\Delta t$. . . . . . . . . . . . . . . . . . . . 35 
4.4. En la Figura (a), graficamos los máximos del $S D R_{r}(\Delta t)$ como función del tiempo entre el primer pulso y el segundo $(\Delta t)$ en ausencia de campo magnético. Notamos que los máximos de $S D R_{r}(\Delta t)$ muestran el flip-flop de espín electrón-núcleo de los centros paramagnéticos, la frecuencia de oscilación es $\omega_{I H F} \approx 23.7 \mathrm{GHz}$. Dicha frecuencia corresponde a la energía de la constante hiperfina $\mathcal{A}=704 \times 10^{-4} \mathrm{~cm}^{-1}$. Con el fin de explicar las fluctuaciones en el filtrado de espín, en la Figura (b) mostramos dos situaciones extremas en la polarización electrónica de las trampas. Las curvas azul y punteada representan las polarizaciones electrónica y nuclear, respectivamente, de las trampas no apareadas debido al primer pulso $\left(P_{0}\right)$. En la primera situación, incidimos un pulso-prueba $\left(P_{1}\right)$ cuando la polarización electrónica de las trampas es máxima y en la segunda situación incidimos un pulso-prueba $\left(P_{2}\right)$ cuando la polarización es mínima. La máxima polarización significa que los electrones en las trampas están alineados en la misma dirección que excitamos inicialmente. En cambio,

cuando la polarización es mínima los electrones de las trampas están polarizados en la dirección opuesta. Los pulsos $P_{0}, P_{p 1}, P_{p 2}$ inyectan espín en la misma dirección. En la primera situación el filtrado es máximo ya que los pocos electrones con polarización de espín opuesta a la dirección de inyección inicial se recombinan rápidamente en las trampas así que la polarización en la BC aumenta. En la segunda situación la situación es totalmente opuesta por lo que el filtrado es mínimo y la polarización en la $\mathrm{BC}$ disminuye. . . . . . . . . . . . . . . . . . . . . . 37

4.5. En la figura (a) mostramos los resultados experimentales 2 de la propuesta 1 1 . Cada círculo azul representa el máximo de la $S D R_{r}$ del pulso prueba como función del tiempo de retardo $(\Delta t)$ entre el pulso inicial y el pulso prueba . Las mediciones fueron hechas para una potencia del láser $P=4 m W$ y a una temperatura $T=7 K$. El comportamiento de la $S D R_{r}(\Delta t)$ es sinusoidal evanescente. La linea punteada representa el ajuste de los puntos como la combinación lineal de dos funciones cosenos evanescentes ecuación 14.5 Las frecuencias de oscilación son las frecuencias asociadas a las energías de las constantes hiperfinas de los isótopos estables de Galio $\mathrm{Ga}^{69}$ y Ga $^{71}$. Usamos dos tiempos característicos de decaimiento $\tau_{s c}^{*}$ el tiempo relajación del espín electrónico de las trampas no apareadas y $T_{2}^{*}$ el tiempo de coherencia de las oscilaciones. En las Figuras (b) y (c) mostramos los espectros de fotolumniscencia para tiempos de retado $\Delta t$ de $150 \mathrm{ps}$ y $250 \mathrm{ps}$, respectivamente. Notamos que las intensidades de los espectros cambian, esto se debe a las fluctuaciones del filtrado debido a la interacción hiperfina de los centros paramagnéticos 2. . . . . . . . . . . . . . . . . 39 
4.6. Los puntos azules representan los datos experimentales para un intervalo de $\Delta t[0,1000]$ ps. Las líneas azul y roja son funciones senoidales que representan las oscilaciones de la superposición de los dos isótopos $\mathrm{Ga}^{69}$ y $\mathrm{Ga}^{71}$ sin amortiguamiento en las configuraciones $\mathrm{Ga}_{i}-C$ y $\mathrm{Ga}_{i}-D$, respectivamente. Las líneas punteadas representan las funciones senoidales amortiguadas descritas por $T_{2}^{*}$. Las líneas verticales punteadas señalan la posición de los máximos y mínimos de los puntos experimentales. . . . 41

4.7. Razón de recombinación dependiente del espín en función del tiempo de retardo $(\Delta t)$ entre el pulso y el pulso-prueba. En el panel (a), los círculos representan los resultados experimentales y en el panel (b), los triángulos representan los resultados de nuestro modelo (Apéndice B). Las figuras de color azul son la $S D R_{r}(\Delta t)$ para $B=0$ y las figuras de color morado son la $S D R_{r}(\Delta t)$ para $B=65 \mathrm{mT}$. Las líneas punteadas en las dos figuras representan el ajuste de los resultado experimentales ( eucación 4.5) para \begin{tabular}{|c}
\hline el caso en que $B=0$. Los puntos experimentales y los calculados por el \\
\hline modelo tienen las mismas intensidades y el mismo comportamiento para
\end{tabular} \begin{tabular}{l}
\hline el caso en que $B=0$. Los puntos experimentales y los calculados por el \\
\hline modelo tienen las mismas intensidades y el mismo comportamiento para
\end{tabular} los casos con y sin campo magnético. . . . . . . . . . . . . . . . . . 42

4.8. La polarización de espín de banda de conducción creado por el pulso prueba como función del tiempo de retardo $\Delta t$ entre el pulso inicial y el pulso prueba para $P=4 \mathrm{~mW}$. (a) Grado de polarización Pez como una función de $\Delta t$. (b) Las componentes transversales del grado de polarización de espín Pex y Pey como función de $\Delta t$. . . . . . . . . . . . . . 44

4.9. Grado de polarización de espín $P_{e z}$ creado por el pulso prueba como función del tiempo de retraso $\delta t$ para diferentes combinaciones de la potencia del pulso y del pulso prueba para $B=0$. (a) $P=4 \mathrm{~mW}$ y $\alpha=1$, (b) $P=40 \mathrm{~mW}$ y $\alpha=1$, (c) $P=0.4 \mathrm{~mW} \mathrm{y} \alpha=100$, (d) $P=40 \mathrm{~mW} \mathrm{y}$

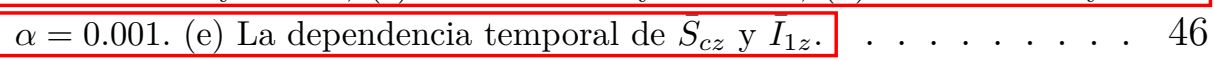

4.10. Diferencia entre los máximos y mńimos de las curvas de grado de polarización de espín Pez de la Figra 4.9 . Los puntos rojos, negros, azules y naranjas son los casos en que $\alpha=1$ y $P=40 \mathrm{~mW}, \alpha=1$ y $P=4 \mathrm{~mW}$ $\alpha=100$ у $P=4 \mathrm{~mW}$, y $\alpha=0.001$ у $P=4 \mathrm{~mW}$. . . . . . . . . . . . 47

4.11. Grado de polarización de espín para las tres componentes espaciales $P_{e z}$,

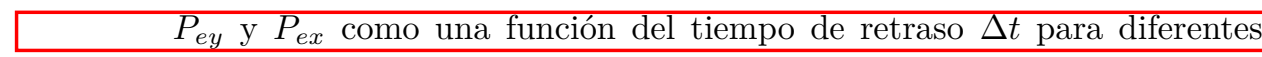
combinaciones de la potencia del pulso y el pulso prueba para $B_{x}=$ 10mT. (a) $P=4 \mathrm{~mW}$ y $\alpha=1$, (b) $P=40 \mathrm{~mW}$ y $\alpha=1$, (c) $P=0.4 \mathrm{~mW}$ y $\alpha=100$, (d) $P=40 \mathrm{~mW}$ y $\alpha=0.001$. (e) La dependencia de temporal de las tres componentes espaciales de espín electrónico $\bar{S}_{c z}, \bar{S}_{c y}, \bar{S}_{c x} \mathrm{y}$

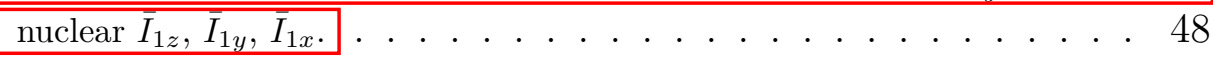


4.12. Diferencia entre los máximos y mńimos de las curvas de grado de polarización de espín Pez, Pey y Pex de la Figra 4.9| Los puntos rojos, negros, azules y naranjas son los casos en que $\alpha=1$ y $P=40 \mathrm{~mW}, \alpha=1$ y $P=4 \mathrm{~mW}, \alpha=100$ y $P=4 \mathrm{~mW}$, y $\alpha=0.001$ y $P=4 \mathrm{~mW} . \quad \ldots . . . \quad 49$

4.13. Comparación de la polarización $P_{e x}$ en configuración de Voigt cuando|IHF es cero (circulos rojos) y cuando tomamos en cuenta la interacción hiperfina. . . . . . . . . . . . . 50

4.14. Grado de polarización de espín Pex como una función del tiempo de retraso $\Delta t$ entre el pulso y el pulso prueba para varios orientaciones del campo magnético. Los ángulos son medidos con respecto a la dirección de luz incidente. Las curvas para las orientaciones del campo magnético $\theta_{B}=30^{\circ}, 45^{\circ}, 60^{\circ}, 90^{\circ}$ son mostradas. Todas las curvas son calculadas para $B=10 \mathrm{mT}, P=40 \mathrm{~mW}$ y $\alpha=1$. El inset muestra $P$ ex para tiempos de retraso más largos. . . . . . . . . . . . . . . . . . . . . 51

4.15. Polarización $P_{e x}$ en presencia de un campo magnético externo en con un angulo de inclinación de $\theta=45^{\circ}$ con referente a la dirección de polarización. El comportamiento oscilante de la curva naranja se debe al flip-flop de los centros paramagnéticos debido al acomplamiento hiperfino del espín nuclear y el espín electrónico de las trampas paramagnéticas. . . . . . . . . 52 


\section{Introducción}

$\mathrm{E}$ l desarrollo de nuevos dispositivos con aplicaciones en espintrónica y computación cuántica ha impulsado la busqueda de nuevas y poderosas herramientas experimentales y teóricas para estudiar las características del espín de los sistemas de estado solido.

Algunas de las propuestas más prometedoras para implementar los registros cuánticos consisten en arquitecturas basadas en el espín nuclear [6]. En este tipo de configuraciones el espín nuclear de un defecto puntual está acoplado a un espín electrónico a través de la interacción hiperfina. Dado que el espín nuclear tiene un alto grado de aislamiento, los espines nucleares interactúan con el medio ambiente y el campo electromagnético únicamente a través del espín electrónico ligado [7]. Aunque estos sistemas crean bits cuánticos muy estables, abordar los estados nucleares es muy complicado. Este tipo de configuraciones se han encontrado en una alta gama de defectos puntuales como en el diamante [7, 8], impurezas de Nitrogeno en diamante con vacancias [9-14], impurezas de Fósforo en el Silicio [15-20], vacancias en el Carburo de Silicio [21] y más recientemente impurezas de Germanio en diamante con vacancias [22]24].

Las técnicas basadas en resonancia magnética nuclear proporcionan los medios para controlar los estados nucleares [8] y sondear los principales mecanismos de polarización nuclear [25-30]. Sin embargo, estas técnicas tienen una muy baja sensibilidad de detección causada principalmente por la débil magnetización de los espines nucleares [31. Además, los métodos desarrollados para mejorar su sensibilidad generalmente implican temperaturas criogénicas e intensos campos magnéticos.

Existen métodos alternativos que permiten la resolución temporal y alta sensibilidad. La respuesta temporal de la rotación de Faraday ha sido usada para estudiar la evolución del espín. La rotación de Faraday es el fenómeno por el cual el plano de la polarización lineal de un láser dirigido a lo largo de una dirección $\vec{k}$ en un medio magnetizado es rotado. Esta rotación se debe a los diferentes índices de refracción que tiene el medio. Estos índices dependen 
de la densidad neta del espín de los portadores de carga en cada región del medio. Por lo tanto la rotación de Faraday da información de la polarización promedio del espín en el medio magnetizado.

Algunos de los sistema estudiados con la rotación de Faraday son: la relajación del espín en pozos cuánticos magnéticos [32], la dinámica del espín en los cristales iónicos YAG Ce ${ }^{3+}$ [33, 34], la preseción del espín en ortoferritas [35] y la coherencia del espín en puntos cuánticos en CdS coloidal [36].

Además, se ha mostrado que los esquemas de pulso-prueba son capaces de dar la respuesta temporal de la dinámica del espín de materiales antiferromagnéticos mediante la detección de los cambios de birrefringencia inducidos por el pulso [37]. También, se ha observado la relajación del espín de átomos individuales utilizando un esquema de pulso-prueba electrónico, el cual combina la microoscopía de efecto túnel y el barrido de polarización de espín [38].

En la última década se han realizado diferentes trabajos experimentales con el fin de demostrar que los semiconductores de GaAsN son excelentes candidatos para las aplicaciones en espintrónica. ([39-42]). Dichos trabajos muestran que los semiconductores de GaAsN, pueden proporcionar los medios para superar las limitaciones en el filtrado y la detección de la polarización de espín a temperatura ambiente en ausencia de campos magnéticos. Esto se debe a que los semiconductores nitrurados alcanzan elevados grados de polarización con tiempos de relajación grandes, a diferencia de los semiconductores magnéticos que lo hacen a bajas temperaturas e intensos campos magnéticos.

Las características peculiares del GaAsN se deben principalmente al mecanismo de recombinación dependiente del espín via los centros paramagnéticos de $\mathrm{Ga}^{2+}$. Los estados de los centros paramagnéticos se encuentran entre las bandas de conducción y de valencia. El mecanismo de recombinación dependiente del espín es el responsable de la polarización de los electrones de la banda de conducción y de los electronces de los centros paramagnéticos [4, 5]. La polarización de los electrones se debe, por un lado a la captura de electrones de la banda de conducción en los centros paramagnéticos siempre que los espines de los electrones de la banda de conducción y de las trampas sean antiparalelos, por otro lado, al bloqueo de electrones de la banda de conducción a las trampas cuando sus espines son paralelos. De este modo el mecanismo de recombinación dependiente del espín genera una eficiente polarización y un eficiente filtrado de espín.

En trabajos recientes mostramos que la interacción hiperfina entre el espín electrónico y nuclear de las centros paramagnéticos en el GaAsN juega un papel crucial en la polarización electrónica en la banda de conducción y en 
los centros [43]. Resultados similares se anunciaron en la Ref. [40]. Además, vimos que es posible sintonizar la polarización del espín nuclear de los centros con la polarización de los electrones en la banda de conducción por medio del mecanismo de la recombinación dependiente del espín y de la interacción hiperfina [43].

Se ha mostrado que la interacción hiperfina es muy útil en el control del tiempo de coherencia del espín electrónico de las impurezas de Fósforo en el Silicio [16, 17, 19, 20, 44, 45] y de las impurezas de Nitrógeno en el diamante con vacancias [9-13, 46-50]. A diferencia de las impurezas de Nitrógeno en el diamante y los defectos en el Silicio, los defectos intersticiales $\mathrm{Ga}^{2+}$ en el GaAsN se benefician de las características de los centros paramagnéticos profundos y aislados los cuales están inmersos en un sistema semiconductor que opera eléctrica y ópticamente, un ejemplo de esto es el efecto de la fotoconductividad gigante dependiente del espín [42, 51].

En la Ref. [43]. Recientemente estudiamos la dinámica del espín en semiconductores de GaAsN incluyendo la interacción hiperfina con el enfoque de la ecuación maestra en régimen de onda continua, similares resultados se reportaron en la Ref. [52. Sin embargo se ha mostrado en la Ref. [53] que el bombeo óptico es de gran interés en aplicaciones de memorias ópticas.

En este trabajo, desarrollamos un modelo que describe la dinámica del espín del GaAs dopado con Nitrógeno en régimen pulsado. Los objetivos principales de este trabajo son trazar y detectar el flip-flop del espín electrónnúcleo en las trampas paramagnéticas en el GaAsN en régimen pulsado. Para cumplir con nuestro cometido, proponemos un modelo basado en el enfoque de la ecuación maestra para la matriz de densidad que incorpora las principales interacciones entre los electrones de la banda de conducción, los huecos de la banda de valencia y las trampas paramagnéticas. Este modelo supera la mayor parte de las limitaciones que tienen los modelos previos [43, 52]. Primero, considera todos los estados nucleares de los centros paramagnéticos de $\mathrm{Ga}^{2+}$ con espín $J=3 / 2$. Segundo, toma en cuenta la interacción hiperfina entre los espines electrónico y nuclear de los centros paramagnéticos. Tercero, toma en cuenta la inyección de espín en el GaAsN. Cuarto, introduce los mecanismos de recombinación dependiente del espín a partir del modelo de dos cargas. Quinto, el modelo es tridimensional por lo que nos proporciona un mejor entendimiento de la dinámica del espín ya que la interacción hiperfina en los centros paramagnéticos acopla las tres direcciones espaciales del espín. Sexto, considera los mecanismos de relajación del espín nuclear de las trampas. Con los resultados obtenidos de la dinámica del espín en régimen pulsado, proponemos un esquema de pulso-prueba óptico que nos permite trazar y detectar el flip-flop del espín electrón-núcleo de los centros 
paramagnéticos en el GaAsN [1]. Las predicciones del modelo fueron confirmadas posteriormente [2]. Estos resultados experimentales muestran que los procesos de polarización y de filtrado de espín tienen fluctuaciones debido al flip-flop inducido por la interacción hiperfina en los centros paramagnéticos. Las fluctuaciones en el filtrado de espín fueron observadas a través de la intensidad de la fotoluminiscencia y la razón de recombinación dependiente del espín. Es importante notar que el esquema de pulso-prueba propuesto es totalmente óptico y no requiere de un campo magnético externo ni el uso de microondas. Hasta ahora ha sido probado que es posible observar estas oscilaciones midiendo la razón de recombinación dependiente del espín. Sin embargo, dicha razón no tiene resolución espacial por lo que no permite observar la dinámica del espín en las tres direcciones a través de su medición. Para finalizar, mostramos que es posible trazar y detectar el flip-flop en las tres componentes espaciales de la polarización por medio de una adaptación a la propuesta [1]. Logramos esto introduciendo un campo magnético oblicuo a la dirección en la que se polarizan los electrones de la banda de conducción. A pesar de que el campo magnético induce su propia dinámica del espín, algunas características observadas pertenecen exclusivamente a las relajaciones del espín y a la interacción hiperfina [3]. Mostramos cuales son las condiciones óptimas del experimento para detectar y trazar el flip-flop del espín electrón-núcleo en las tres direcciones espaciales.

Con el fin de ilustrar cual es el estado del arte en el estudio de la dinámica del espín del GaAsN, en el segundo capítulo presentamos un resumen sobre las trampas paramagéticas, la importancia de estas trampas en los efectos de polarización y de filtrado del espín en el GaAsN, asimismo hablamos de los principales trabajos de la dinámica del espín en el GaAsN a través del enfoque de la ecuación maestra.

Después, en el tercer capítulo mostramos el desarrollo del modelo. Con el fin de dar una imagen realista del sistema, el modelo toma en cuenta los electrones de la banda de conducción, los huecos de la banda de valencia, los electrones ligados y los núcleos de las trampas paramagnéticas $\left(\mathrm{Ga}^{2+}\right)$. Describimos al sistema mediante un espacio vectorial de operadores que forman un álgebra de Lie finita de dimensión 85 [54]. También consideramos la mayoría de las interacciones de estos cuatro elementos que se manifiestan en los procesos de recombinación, la interacción hiperfina entre el espín electrónico y nuclear de las trampas paramagéticas, y las interacciones de Zeeman de los electrones en la banda de conducción y del electrón ligado en las trampas paramagnéticas.

El cuarto capítulo lo dividimos en tres partes. En la primera parte presentamos los resultados teóricos del modelo considerando un isótopo de $\mathrm{Ga}^{2+}$ 
en régimen pulsado. A través de estos resultados, formulamos la propuesta experimental para detectar el flip-flop de espín electrón-núcleo [1]. Además explicamos en que consiste la propuesta del esquema de pulso-prueba. En la segunda parte, mostramos los resultados teóricos y experimentales para un sistema de mayor complejidad el de dos isótopos de $\mathrm{Ga}^{2+}$ [2]. Estos resultados experimentales corroboraron las predicciones del modelo propuesto en [1. Para finalizar esta sección, en la tercera parte presentamos los resultados del modelo en régimen pulsado en presencia de un campo magnético oblicuo a la dirección de polarización. Es importante notar que uno de los principales objetivos de este trabajo es formular un conjunto de parámetros óptimos para el desarrollo experimental del trazado de las polarizaciones de espín en las tres direcciones espaciales. Así que en esta tercera parte mostramos diferentes pruebas para varios valores de la intensidad de la potencia del pulso-prueba y diferentes ángulos de inclinación del campo magnético.

Para finalizar este trabajo, en el quinto capítulo damos las conclusiones y perspectivas. 


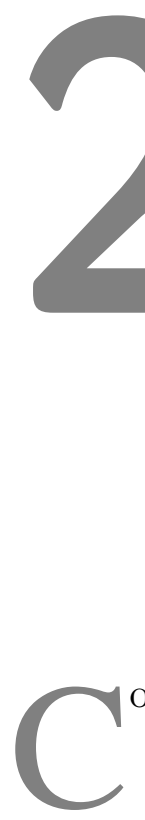

\section{Marco teórico}

es trazar el flip-flop del espín electrón-núcleo en las trampas paramagnéticas del GaAsN en régimen pulsado. Para este propósito, en este capítulo introducimos y comentamos sobre las trampas paramagnéticas en el GaAsN, su importancia de estas trampas en los procesos de filtrado y de polarización del espín en el GaAsN, e importancia del proceso de flip-flop del espín electrón-núcleo. También hablamos sobre el enfoque de la ecuación maestra y los diferentes trabajos sobre el GaAsN usando este enfoque.

\subsection{Trampas paramagnéticas en GaAsN}

Trabajos recientes han demostrado que la sustitución de pequeñas cantidades de Nitrógeno en los sitios de aniones en semiconductores como el InGaAs y el GaAs [55] modifica drásticamente las propiedades ópticas y electrónicas de estos materiales ampliamente utilizados en aplicaciones optoelectrónicas [56, 57].

Para el caso del GaAsN, el Nitrogeno mueve al Galio a los intersticios de la red formando estados isolocalizados [39], que son resonantes con la banda de conducción y están fuertemente unidos a los distintos estados de esta banda [56-59]. Estos estados, también son llamados trampas paramagnéticas o centros de recombinación paramagnéticos. Estas trampas son responsables de la polarización de los electrones en la banda de conducción (BC) y son la causa de que se presente el mecanismo de recombinación dependiente del espín (SDR por sus siglas en ingles Spin Dependent Recombination) entre la BC y la banda de valencia (BV).

Se dice que los Galios intersticiales son centros paramagnéticos en el GaAsN ya que cada Galio tiene un electrón sin aparear en el espín dando 
lugar a un momento magnético que puede alinearse con un campo magnético externo. Estos Galios también son llamados $\mathrm{Ga}^{2+}$ ya que perdieron dos electrones cuando fueron trasladados a los intersticios de la red pasando de una configuración electrónica $4 s^{2} p^{1}$ a $4 s^{1}$ [39].

Los centros paramagnéticos son responsables de la recombinación dependiente del espín (SDR). Esto se debe a que la recombinación entre los electrones de la BC y los centros paramagnéticos, se lleva a cabo solo en el caso de que la proyección de espín de los electrones de la $\mathrm{BC}$ es opuesta a la del espín electrónico de los centros. La naturaleza de esta recombinación esta basada en el principio de exclusión de Pauli [60, 61] que prohíbe a dos fermiones ocupar con los mismos números cuánticos el mismo nivel electrónico.

El mecanismo de la SDR consiste en los siguientes pasos: Primero, excitamos electrones de la $\mathrm{BV}$ a la $\mathrm{BC}$, por lo que se genera una densidad de electrones con polarización neta de espín diferente de cero $(S \neq 0)$ en la $\mathrm{BC}$ Figura 2.1 (a). Para este trabajo consideramos que los huecos se despolarizan muy rápido, por lo que la $\mathrm{BV}$ no se polariza. Segundo, los fotoelectrones generados en la $[\mathrm{BC}$ son atrapados en los centros paramagnéticos, siempre y cuando el espín de los electrones de la $\mathrm{BC}$ sea opuesta al espín electrónico de las trampas, formando, junto con los electrones de las trampas, pares de electrones 2.1 (b). Tercero, uno de los dos electrones apareados en las trampas se recombina con un hueco de la BV dejando un electrón no apareado en la trampa paramagnética 2.1 (c). La primera parte del proceso de recombinación se lleva acabo en un tiempo mucho menor ( entre 1 ps y 150 ps ) que la segunda (1 ns); los centros son saturados rápidamente después se vacían y se polarizan en la misma dirección que los electrones de la BC Figura 2.1 (d), esto impide la recombinación de electrones de la banda de conducción polarizados en una dirección y favorece la recombinación de los electrones polarizados en la $\mathrm{BC}$ en la dirección contraria [51]. Este mecanismo ha sido observado en diferentes sistemas como Silicio dislocado [62, 63], Silicio amorfo [63, 64] y en centros paramagnéticos profundos en GaAs. [4, 65].

En trabajos recientes mostramos que la interacción hiperfina (IHF) entre el espín electrónico y nuclear de las centros paramagnéticos en el GaAsN juega un papel crucial en la polarización electrónica de la banda de conducción y de los centros [43]. Resultados similares se anunciaron en la Ref. [40]. Además, vimos que es posible sintonizar la polarización del espín nuclear de los centros con la polarización de los electrones en la banda de conducción por medio del mecanismo de la recombinación dependiente del espín y de la interacción hiperfina [43]. La energía debido a la IHF en el GaAsN es del orden de $15 \mu \mathrm{eV}[2]$.

La $\llbracket \mathrm{IHF}$ representa un mecanismo clave en los sistemas semiconductores.

UAM-Iztapalapa

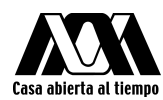




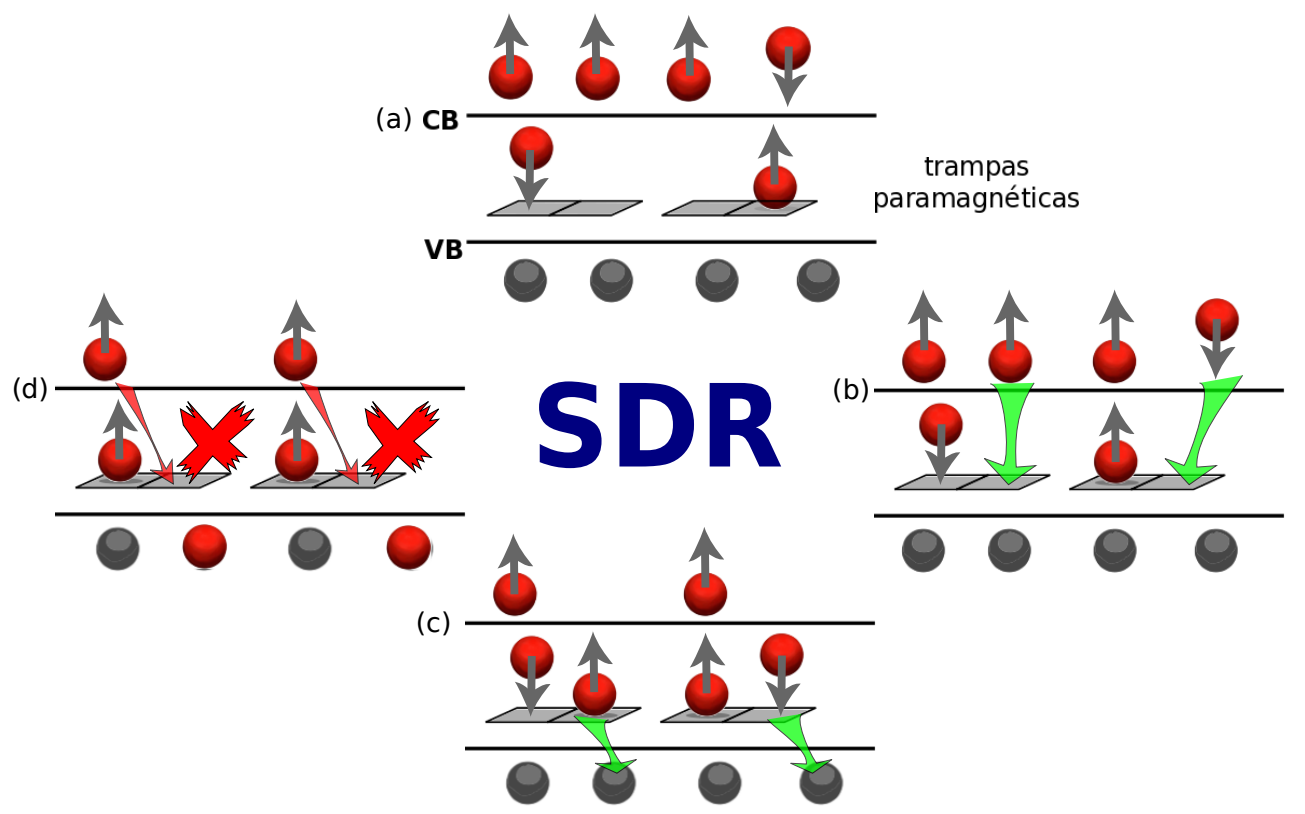

Figura 2.1: El mecanismo de la SDR consiste en cuatro pasos: Primero, excitamos electrones de la $\mathrm{BV}$ a la $\mathrm{BC}$ la polarización neta de espín es diferente de cero $(S \neq 0)$ en la BC 2.1 (a). Segundo, los fotoelectrones en la BC caen en los centros paramagnéticos, siempre y cuando el espín de los electrones de la BC sea opuesta al espín electrónico de las trampas, formando, junto con los electrones de las trampas, pares de electrones 2.1 (b). Tercero, uno de los dos electrones apareados en las trampas cae en un hueco de la BV 2.1 (c). La primera parte del proceso de recombinación se lleva acabo en un tiempo mucho menor que la segunda; los centros son saturados después se vacían y se polarizan en la misma dirección que los electrones de la BC 2.1 (d)

Este acoplamiento es responsable de la creación de estados mezclados que dependen de los espines electrónico y nuclear. Además, se sabe que la polarización nuclear de las impurezas en un semiconductor puede modificarse debido al proceso de flip-flop del espín electrón-núcleo consecuencia de la IHF [66]. El proceso del flip-flop es el cambio simultaneo de las polarizaciones de los espines electrónico y nuclear los cuales están acoplados mediante la interacción hiperfina. Debido a la IHF y al proceso de flip-flop entre los espines electrónico y nuclear se han propuesto registros cuánticos en el diamante [67.

Así mismo, se ha mostrado que la IHF es muy util para la transferencia de espín electrón-núcleo en el control del tiempo de coherencia del espín electrónico de las impurezas de Fosforo en el Silicio [16, 17, 19, 20, 44, 45] y en las impurezas de Nitrógeno en el diamante con vacancias [9-13, 46-50]. 
Debido a la importancia de esta interacción en el GaAsN y en otros semiconductores con impurezas, es muy importante tener las técnicas óptimas para estudiarla y un modelo que describa su evolución temporal. Una de las formas más convenientes de modelar un sistema semiconductor tomando en cuenta la interacción hiperfina es con el enfoque de la ecuación maestra. En la siguiente sección mostramos en qué consiste el enfoque de la ecuación maestra.

\subsection{Enfoque de la ecuación maestra}

La gran mayoria de los sistemas que se encuentran en la naturaleza son abiertos. Es decir, son sistemas que intercambian energìa y/o materia con su entorno. Por tanto, en el estudio de un sistema cuántico, es muy importante considerar la influencia que tiene el entorno sobre el sistema dinámico en estudio. Los sistemas cuánticos cerrados son descritos por la ecuación de von Neumann 68.

$$
\frac{d \hat{\rho}}{d t}=\frac{1}{i \hbar}[\hat{H}, \hat{\rho}]
$$

donde $\hat{\rho}$ es la matriz de densidad, los elementos $\rho_{m, m}$ son las probabilidades de ocupación de un estado y $\rho_{m, n}(m \neq n)$ son los términos de coherencia. Estos sistemas no tienen interacción con su entorno.

Por otro lado, los sistemas cuánticos abiertos son mejor entendidos a través de la ecuación maestra

$$
\hat{\dot{\rho}}=\frac{1}{i \hbar}[\hat{H}, \hat{\rho}]+D(\hat{\rho})
$$

donde $\hat{\rho}$ es la matriz de densidad, $\hat{H}$ es el hamiltoniano del sistema y $D(\hat{\rho})$ es el disipador. En el disipador se encuentran todos los procesos que hay entre el sistema y sus alrededores.

En la Ref. [43], mostramos que la polarización de espín nuclear de los defectos de $\mathrm{Ga}^{2+}$ puede ser modificada mediante la óptima polarización de espín de los electrones de la $\mathrm{BC}$ por bombeo óptico en régimen de onda continua (CW). El disipador está construido como una combinación lineal de elementos de un espacio ortogonal de operadores. Dicho espacio está formado por 16 generadores ortogonales con la traza. La ecuación maestra está constituida por un sistema de 144 ecuaciones diferenciales no lineales acopladas. Cabe mencionar que en este primer modelo no tomamos en cuenta las perdidas debidas a la relajación de las trampas paramagnéticas apareadas. 
En la Ref. [52], presentaron un estudio teórico de la orientación óptica del espín nuclear y electrónico y la recombinación dependiente del espín en presencia de campo magnético longitudinal. Para este trabajo consideraron el acoplamiento hiperfino entre el espín de los electrones ligados y el espín nuclear de los centros paramagnéticos. La ecuación maestra para el sistema incluye la relajación de espín nuclear usando dos tiempos diferentes de relajación $\tau_{n 1}$ para las trampas no apareadas y $\tau_{n 2}$ para las apareadas. La ecuación maestra está formada por 21 ecuaciones fenomenologicas acopladas provenientes del modelo de dos cargas $([4,5]$ ). En este caso, el modelo propuesto solo considera las ecuaciones diferenciales que tienen que ver con los elementos diagonales de la matriz de densidad, Además se considera que el espín nuclear de los centros es $J=1 / 2$ y utilizan bombeo óptico en régimen continuo.

En estos dos trabajos no consideran el bombeo óptico en régimen pulsado [43, 52. Como se dijo antes, este régimen es de gran interés en aplicaciones de memorias ópticas [69].

En este trabajo, logramos unir las ventajas de los dos trabajos previos [43, 52. Obtuvimos un modelo utilizando el enfoque de la ecuación maestra tridimensional que tiene seis características principales. Primero, la base de operadores que describe al sistema forma un álgebra de Lie de dimensión finita. Segundo, el modelo describe la inyección de espín por incidencia de luz en el GaAsN. Tercero, los coeficientes que describen la dinámica no son constantes y están íntimamente ligadas con el modelo de dos cargas [4, 5]. Cuarto, el modelo considera la interacción hiperfina entre el espín electrónico y nuclear de los centros de $\mathrm{Ga}^{2+}$. Quinto, tomamos en cuenta todos los estados nucleares de los centros de $\mathrm{Ga}^{2+}$ con espín $J=3 / 2$. Sexto, incluimos los mecanismos de relajación del espín electrónico y nuclear. En las siguientes secciones describimos las primeras tres características del modelo.

\subsection{1. Álgebras de Lie}

Se dice que un conjunto de operadores $\hat{h}_{1}, \hat{h}_{2}, \ldots, \hat{h}_{n}$ forman un álgebra de Lie finita $\mathcal{L}_{n}$, si el conmutador de dos elementos cualesquiera de $\mathcal{L}_{n}$ puede ser expresado como una combinación lineal de los elementos de $\mathcal{L}_{n}$

$$
\left[\hat{h}_{i}, \hat{h}_{j}\right]=i \hbar \sum_{k=1}^{n} C_{i, j, k} \hat{h}_{k}
$$

donde las constantes de estructura $C_{i, j, k}$ contienen toda la informaciòn concerniente del grupo unitario generado por $\mathcal{L}_{n}$. Se dice que un operador $\hat{O}$ tiene un algebra dinámica si este puede ser expresado como una combinación 
lineal de los elementos de $\mathcal{L}_{n}$

$$
\hat{O}=\sum_{k=1}^{n} a_{k}(t) \hat{h}_{k}
$$

donde los coeficientes $a_{k}$ son en general funciones del tiempo. Este enfoque lo utilizamos para solucionar hamiltonianos dependientes del tiempo en la Ref. ([54]). En este trabajo utilizamos las álgebras de Lie para representar los operadores de la matriz de densidad $(\hat{\rho})$, del hamiltoniano $(\hat{H})$ y del disipador $D(\hat{\rho})$ en la forma $(2.4)$.

\subsubsection{Inyección de espín en GaAsN}

La técnica de bombeo óptico es una forma conveniente de inyectar espín en el GaAsN. Esta técnica utiliza la luz para llevar a los electrones de un nivel bajo de energía a uno más alto. La cantidad de electrones excitados con cierta proyección de espín dependerá de la frecuencia, polarización e intensidad de la luz incidente.

De acuerdo con las reglas de selección del GaAs, durante el bombeo óptico con luz linealmente polarizada se obtienen poblaciones iguales de electrones fotogenerados en la $\mathrm{BC}$ con espín hacia arriba y hacia abajo por lo que la polarización total de espín es nula en la BC. Por el contrario, al incidir luz circularmente polarizada a la izquierda (derecha) $\sigma^{-}\left(\sigma^{+}\right)$sobre el semiconductor se obtiene $75 \%$ de electrones fotogenerados en la BC con espín hacia abajo (arriba) y $25 \%$ en la dirección contraria [4, 41, 65, 70). Por lo tanto, cuando se incide luz circularmente polarizada, la polarización total del espín en la banda de conducción es diferente de cero.

La diferencia entre las poblaciones de electrones con espín hacia arriba y hacia abajo por medio de la estimulación con luz circularmente polarizada, se debe a las particulares reglas de selección que rigen las transiciones permitidas entre la BC] y la BV en el GaAs.

Para el GaAs el punto más alto de todas las bandas de valencia, se encuentra localizado en el centro de la primera zona de Brillouin $(\Gamma \equiv 000)$; en éste existe mayor probabilidad de que se den las transiciones entre las bandas. En este punto las bandas de huecos ligeros y pesados tienen degeneración cuadruple. Esto se debe a que los huecos pesados tienen $J=\frac{3}{2}$ con proyecciones $J_{z}= \pm \frac{3}{2}$ y los huecos ligeros tienen $J=\frac{3}{2}$ con proyecciones $J_{z}= \pm \frac{1}{2}$. Por último, los huecos split-off se encuentran en un nivel de energía menor debido al acoplamiento espín-órbita con una energía $E=E_{g}-\Delta_{S O}$ con $J=\frac{1}{2}$ y proyecciones $J_{z}= \pm \frac{1}{2}$.

Las reglas de selección son en parte consecuencia de la conservación del momento angular. En la absorción de un fotón polarizado circularmente, éste 


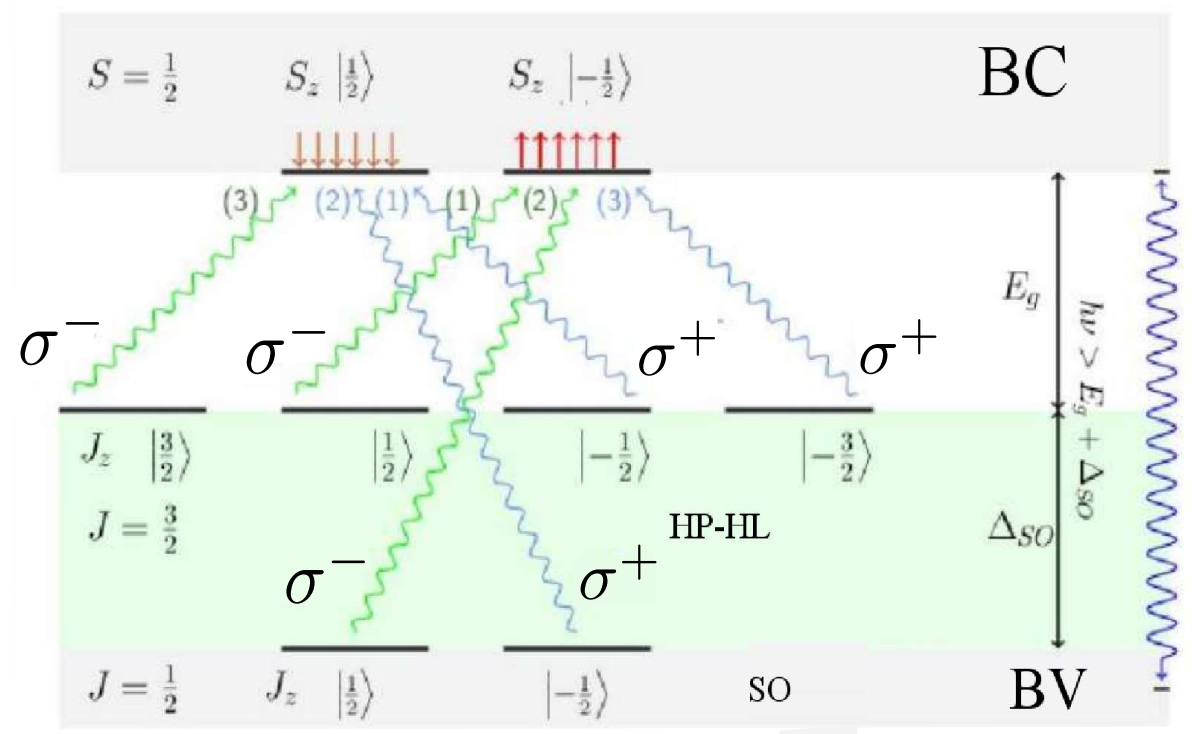

Figura 2.2: Diagrama de las reglas de selección entre la BC y la BV. Las transiciones inducidas por luz circularmente polarizada hacia la izquierda están de color azul mientras que las transiciones debio a la luz circularmente polarizada hacia la derecha están de color verde. La probabilidad de transición de un estado de la BV a la BC o también llamada fuerza de oscilador se indica con un número al lado de cada transición

transfiere un momento angular $L_{z}= \pm 1$ al electrón con cierta proyección de espín por lo que sólo puede haber transiciones de $\Delta L_{z}= \pm 1$.

En la Figura 2.2 mostramos un diagrama de las transiciones permitidas entre los estados de la banda de valencia y conducción. Vemos las transiciones inducidas por luz circularmente polarizada hacia la izquierda en azul mientras que las circularmente polarizadas hacia la derecha son de color verde. La probabilidad de transición de un estado de la BV a la BC o también llamada fuerza de oscilador se indica con un número al lado de cada transición. Por ejemplo, la transición de $J_{z}=3 / 2$ a $S_{z}=1 / 2$ tiene una fuerza de oscilador de 3 mientras que la transición de la banda de huecos ligeros $J_{z}=1 / 2$ a $S_{z}=-1 / 2$ tiene una fuerza de oscilador de 1 . Esto quiere decir que la primera transición es tres veces más probable que la segunda. Por esta razón, al incidir con luz circularmente polarizada tenemos el $75 \%$ de la población de los electrones con la misma dirección del espín y el restante $25 \%$ en la dirección opuesta.

Con el fin de ilustrar las reglas de selección, consideramos el caso para un electrón en la banda de valencia absorbe la energía $\hbar \nu$ de un fotón circularmente polarizado a la izquierda $\left(\sigma^{-}\right)$con $L_{z}=-1$. Éste sólo puede efectuar las siguientes transiciones: 


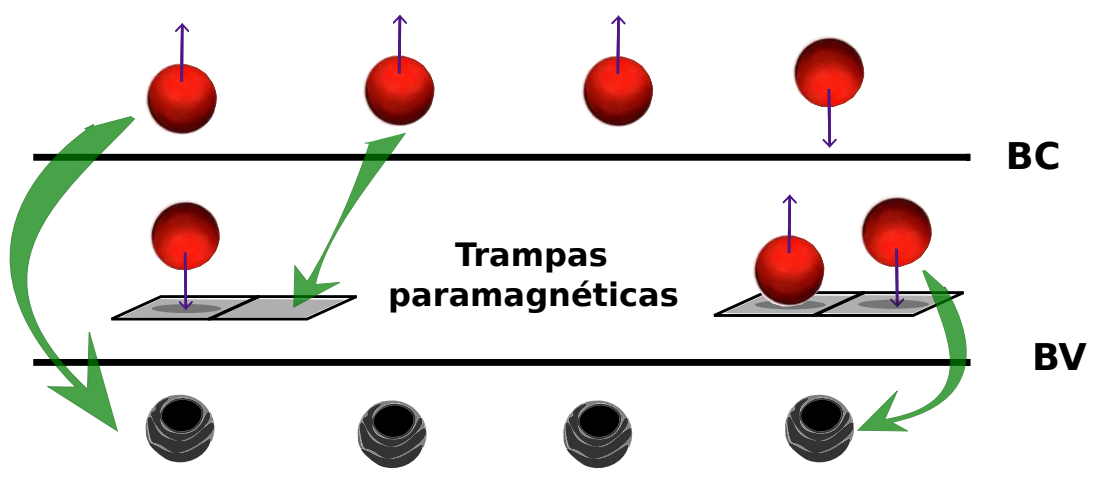

Figura 2.3: Esquema del flujo de la carga entre la banda de conducción, las trampas paramagnéticas y la banda de valencia. La carga fluye desde la banda de conduccón, transita por las trampas paramagnéticas y termina en la banda de valencia. Este flujo de carga está descrito por un conjunto de ecuaciones fenomenológicas denominado modelo de dos cargas. [4, 5]

(1) De los huecos ligeros $J_{z}=-\frac{1}{2}$ a la banda de conducción $S_{z}=\frac{1}{2}$.

(2) De los huecos Split-off $J_{z}=-\frac{1}{2}$ a la banda de conducción $S_{z}=\frac{1}{2}$.

(3) De los huecos pesados $J_{z}=-\frac{3}{2}$ a la banda de conducción $S_{z}=-\frac{1}{2}$.

Por otra parte si un electrón absorbe la energía de un fotón $\hbar \nu$ con polarización circular a la derecha $\sigma^{+}$con $L_{z}=1$, solamente pueden ocurrir las transiciones siguientes:

(1) De los huecos ligeros $J_{z}=\frac{1}{2}$ a la banda de conducción $S_{z}=-\frac{1}{2}$.

(2) De los huecos Split-off $J_{z}=\frac{1}{2}$ a la banda de conducción $S_{z}=-\frac{1}{2}$.

(3) De los huecos pesados $J_{z}=\frac{3}{2}$ a la banda de conducción $S_{z}=\frac{1}{2}$.

\subsubsection{Modelo de dos cargas}

Dado que los coeficientes del disipador están íntimamente ligados al modelo de dos cargas, es necesario decir en que consiste este modelo. El modelo de dos cargas (TCM por sus siglas en ingles Two Charge Model), es un conjunto de ecuaciones fenomenológicas que describen el flujo de la carga de un semiconductor con impurezas paramagnéticas. Este flujo de carga se da entre la BC, las trampas paramagéticas y la BV.

El TCM fue propuesto por Kalevich et al. [4, 5]. Éste modela de una manera clara y sencilla el mencanismo de recombinación dependiente del 
espín en el GaAsN. Este modelo considera a los electrones en la BC, a los huecos en la BV, a las trampas paramagnéticas apareadas y no apareadas. Las trampas no apareadas son aquellos centros de recombinación que solo tienen un electrón en cambio las trampas apareadas tienen dos electrones consecuencia de que un electrón de la $[\mathrm{BC}]$ se recombinó en el centro.

Dicho modelo está formado por un conjunto de ecuaciones de balance para los electrones y la polarización de espín electrónica en la BC; para las trampas paramagnéticas no apareadas y la polarización de espín electrónica de estos centros de recombinación; para las trampas paramagnéticas apareadas y los huecos en la BV] El sistema de ecuaciones está dado por:

$$
\begin{gathered}
\frac{\partial n}{\partial t}=-\frac{\gamma_{e}}{2}\left(n N_{1}-4 \vec{S} \cdot \vec{S}_{c}\right)-\gamma_{r} n p+G, \\
\frac{\partial p}{\partial t}=-\gamma_{h} p N_{2}-\gamma_{r} n p+G \\
\frac{\partial N_{1}}{\partial t}=-\frac{\gamma_{e}}{2}\left(n N_{1}-4 \vec{S} \cdot \vec{S}_{c}\right)-\gamma_{h} N_{2} p \\
\frac{\partial N_{2}}{\partial t}=\frac{\gamma_{e}}{2}\left(n N_{1}-4 \vec{S} \cdot \vec{S}_{c}\right)+\gamma_{h} N_{2} p \\
\frac{\partial \vec{S}}{\partial t}=\frac{\gamma_{e}}{2}\left(\vec{S} N_{1}-\vec{S}_{c} n\right)-\gamma_{r} p \vec{S}+\vec{G}_{s}, \\
\frac{\partial \vec{S}_{c}}{\partial t}=-\frac{\gamma_{e}}{2}\left(\vec{S} N_{1}-\vec{S}_{c} n\right)
\end{gathered}
$$

donde la variable $n$ es la densidad de electrones en la $\mathrm{BC}, \vec{S}$ es el vector de polarización de espín de los electrones en la BC, $p$ es la densidad de huecos en la $\mathrm{BV}, N_{1}$ es la densidad de trampas no apareadas, $\vec{S}_{c}$ es el vector de polarización de espín de los electrones ligados a las trampas no apareadas y $N_{2}$ es la densidad de trampas apareadas. El coeficiente $\gamma_{e}$ es la velocidad de recombinación de los electrones entre la BC y las trampas no apareadas $\left(N_{1}\right), \gamma_{h}$ es la velocidad de recombinación de los electrones entre la trampas apareadas $\left(N_{2}\right)$ y la BV y $\gamma_{r}$ es la velocidad de recombinación bimolecular de los electrones entre la BC y BV. La generación de electron-hueco en la BC y en la BV, respectivamente, esta dado por $G$. Para finalizar, $\vec{G}_{s}$ es la generación de polarización promedio de espín en la BC. Los valores de $\gamma_{e}$, $\gamma_{h}$ y $\gamma_{r}$, dependen principalmente de las concentraciones de Nitrógeno en el GaAsN y de la temperatura.

El mecanismo de la SDR se da entre los electrones de la banda de conducción y las trampas paramagnéticas no apareadas. Los centros paramagnéticos 
poseen un electrón no apareado con cierta orientación del espín de tal suerte que el paso de los electrones dependerá de la orientación relativa de su proyección de espín y la del electrón ligado a los centros de recombinación.

Los téminos $\left(n N_{1}-4 \vec{S} \cdot \vec{S}_{c}\right)$ y $\left(\vec{S} N_{1}-\vec{S}_{c} n\right)$ están asociados con la SDR. Por ejemplo, para el caso en que los electrones de la BC y de las trampas $N_{1}$ tienen la misma dirección de polarización de espín $\left(\vec{S} \cdot \vec{S}_{c}\right)>0$ le velocidad de recombinación de los electrones en la BC se reduce ecuación (2.5). Por otro lado cuando $\left(\vec{S} \cdot \vec{S}_{c}\right)<0$ la velocidad de recombinación aumenta para los electrones en la BC y aumenta la densidad de trampas apareadas $N_{2}$ ecuación (2.8). Notamos que estos dos términos desaparecen cuando el sistema esta completamente polarizado, es decir $S_{z}=n / 2, S_{x}=S_{y}=0, S_{c z}=N / 2 \mathrm{y}$ $S_{c x}=S_{c y}=0$.

A pesar de que este modelo explica muy bien el mecanismo de la SDR, no logra incluir la interacción hiperfina que hay entre el espín electrónico y el espín nuclear de la trampas no apareadas. Ya que el [TCM] no considera al sistema como un sistema cuántico. Es muy importante tomar en cuenta la IHF de las trampas, ya que esta interacción es una de las responsables del comportamiento del tiempo de vida de la polarización de los electrones en la BC y en las trampas $\left(N_{1}\right)$. Además, es importante notar que mediante la IHF podemos sintonizar la polarización nuclear de las trampas con la polarización electrónica en la BC [43]. 


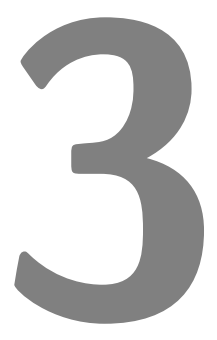

\section{Modelo de la dinámica del espín en GaAsN}

$\mathrm{E}$ n este capítulo, presentamos un modelo utilizando el enfoque de la ecuación maestra para estudiar la dinámica del espín en un semiconductor de $G a A s_{1-x} N_{x}$ en presencia de disipación. La ecuación maestra esta dada por

$$
\frac{\partial \hat{\rho}}{\partial t}=\frac{1}{i \hbar}[\hat{H}, \hat{\rho}]+D(\hat{\rho})
$$

donde $\hat{\rho}$ es la matriz de densidad, $D(\hat{\rho})$ es el disipador y $\hat{H}$ es el Hamiltoniano del sistema. Describimos estas tres cantidades en las siguientes secciones. Aunque es posible incorporar varios isótopos de $\mathrm{Ga}^{2+}$ [39], por simplicidad desarrollamos el modelo solo en la presencia de un isótopo promedio. Esto quiere decir que solo hay un tipo de trampa paramagnética.

\subsection{Matriz de densidad}

El sistema esta formado por huecos $(p)$ en la BV, electrones con cierta proyección de espín en la $\mathrm{BC}(n)$, trampas paramagnéticas apareadas $\left(N_{2}\right)$ y no apareadas $\left(N_{1}\right)$ que están entre la BC y BV. Como se muestra en la Figura 3.1 .

Suponemos que los elementos o subsistemas (electrones, huecos y trampas) que componen al sistema están acoplados solamente por el disipador $(D(\hat{\rho}))$. Por lo tanto, estos subsistemas no comparten términos coherentes. Por consiguiente, expresamos la matriz de densidad del sistema $(\hat{\rho})$ como la suma directa de las $\hat{\rho}$ de cada elemento.

$$
\hat{\rho}=\hat{\rho}_{B V} \oplus \hat{\rho}_{B C} \oplus \hat{\rho}_{N_{1}} \oplus \hat{\rho}_{N_{2}},
$$

donde $\oplus$ representa la suma directa de dos matrices. $\hat{\rho}_{B V}$ y $\hat{\rho}_{B C}$ son las matrices de densidad de la $[\mathrm{BV}$ y de la $\mathrm{BC}$, respectivamente. Las matrices 


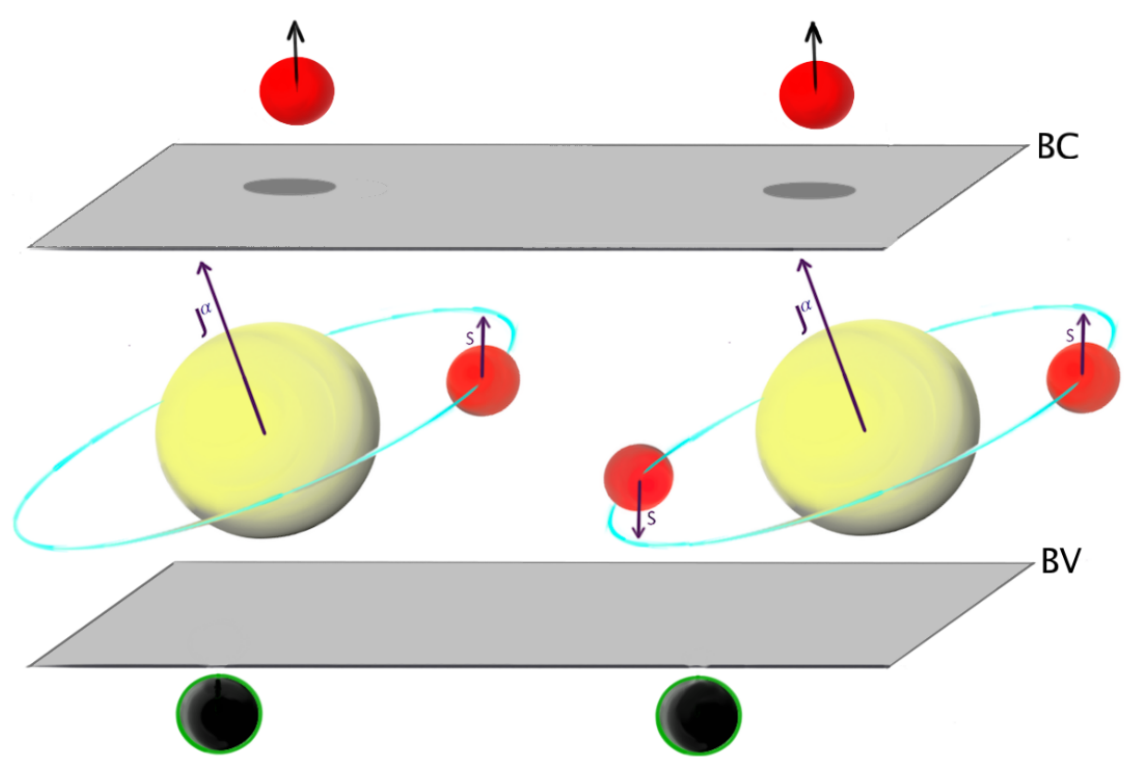

Figura 3.1: La placa superior gris representa la BC donde se encuentran los electrones polarizados. La placa inferior representa la BV donde se encuentran los huecos. Entre las placas están las trampas paramagnéticas apareadas que tienen dos electrones y las no apareadas que tienen un electrón.

$\hat{\rho}_{N_{1}} \mathrm{y} \hat{\rho}_{N_{2}}$ describen a las trampas paramágneticas no apareadas y apareadas, respectivamente. Estas matrices corresponden a los dos diferentes estados de carga del Galio 4s. Donde, los subíndices uno y dos conciernen a los electrones ocupados en el Galio. Mientras el estado de carga descrito por $\hat{\rho}_{N_{1}}$ es estable, $\hat{\rho}_{N_{2}}$ es inestable por consiguiente solo hospeda a un electrón por unos cuantos cientos de picosegundos [41, 55].

Por un lado, consideramos que los huecos se despolarizan muy rapido [71], así que inferimos que solo hay un estado de los huecos en la BV $(\mathfrak{p}=|p\rangle)$. Razón por la cual no tomamos en cuenta los estados de los huecos lijeros y pesados. Por consiguiente, representamos este subsistema con una matriz de $1 \times 1$. Tomamos en cuenta que hay 2 estados en la BC, los fotoelectrones generados con espín hacia arriba $\mathfrak{n}_{+}=|n,+\rangle$ y espín hacia abajo $\mathfrak{n}_{-}=|n,-\rangle$. Así que esta banda esta descrita por una matriz de $2 \times 2$. Ahora bien, los estados de las trampas paramagnéticas no apareados dependen del espín electrónico y del espín nuclear de la trampa. Estos estados $\mathfrak{N}_{ \pm}^{\alpha}=\left|N_{1}, \alpha, \pm\right\rangle$ son 8, están asociados con las dos proyecciones de espín del electrón ligado a la trampa (subíndice \pm ) y las cuatro proyecciones de espín nuclear $(\alpha=$ $-3 / 2,-1 / 2,1 / 2,3 / 2)$. Por ejemplo, $\mathfrak{N}_{+}^{-3 / 2}$ es el estado para la trampa con 
proyección de espín electrónico hacia arriba y con proyección de espín nuclear $-3 / 2$. De este modo estos centros son descritos por una matriz de $8 \times 8$.

Por el contrario, las trampas apareadas solo tienen cuatro estados. Estos estados dependen de la proyección del espín nuclear del centro $\mathfrak{N}_{2}^{\alpha}=\left|N_{2}, \alpha\right\rangle$, donde $\alpha$ puede tomar cuatro valores como en el caso de las trampas no apareadas. Así, este subsistema está definido por una matriz de $4 \times 4$.

Por tanto, la matriz de densidad del sistema es una matriz de bloques como se muestra en el Cuadro 3.1.

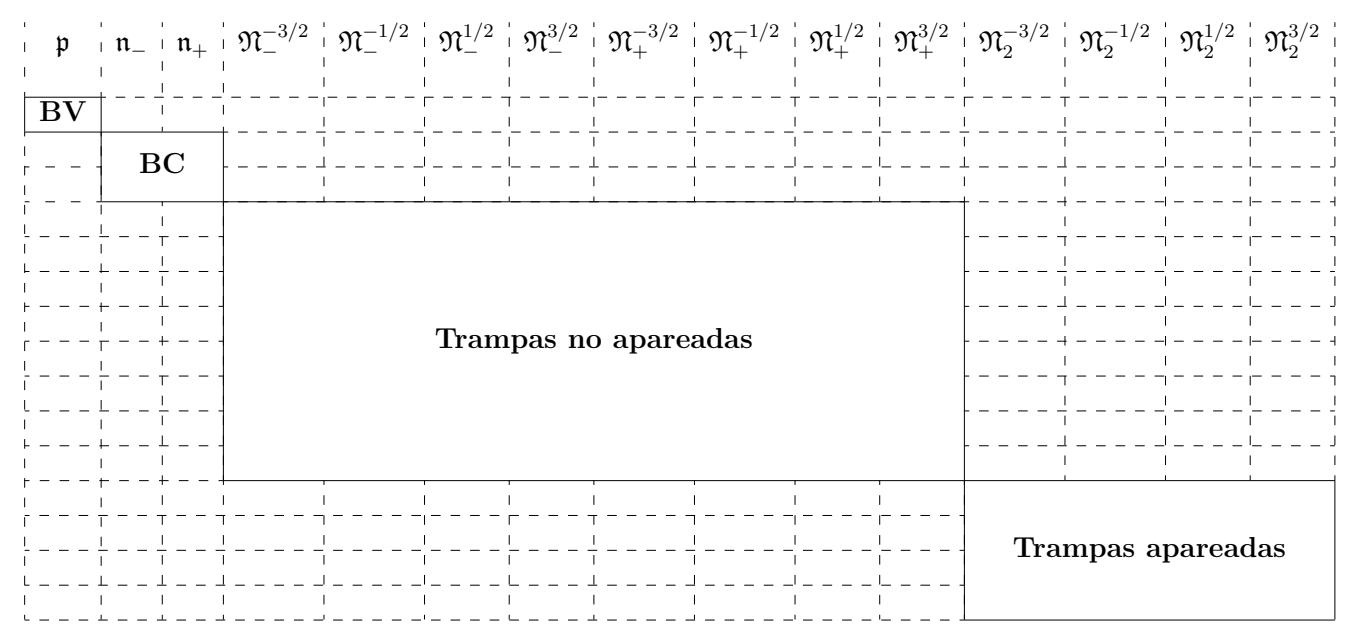

Cuadro 3.1: La matriz de densidad del sistema es una matriz diagonal por bloques. Dicha matriz está formada por los bloques que representan los espacios de la BV, la BC, las trampas no apareadas y las trampas apareadas.

En la siguiente sección mostramos el espacio vectorial de operadores que describe el sistema total.

\subsection{Espacio prehilbertiano}

Con el fin de describir el sistema, construimos un espacio prehilbertiano o espacio vectorial de operadores donde el producto interno es la traza de cualesquiera dos operadores de ese espacio $\left(\langle A, B\rangle \equiv \operatorname{Tr}\left[\mathbf{A}^{T} \mathbf{B}\right]\right)[72]$. Esto lo hacemos con el fin de escribir la matriz de densidad del sistema como una combinación lineal de los generadores que describen a la $\mathrm{BC}$, a la $\mathrm{BV}$. a las trampas apareadas y a las trampas no apareadas. Elegimos a la traza de dos generadores como el producto interno de este espacio ya que el valor esperado mecano-estadístico de un operador $\hat{O}$ lo calculamos como $\operatorname{Tr}[\hat{O} \hat{\rho}]$. 
Por lo que la traza extrae la información las cantidades físicas de la matriz de densidad.

El espacio vectorial del sistema total es

$$
\Lambda=\left\{V_{B V}, V_{B C}, V_{N_{1}}, V_{N_{2}}\right\}
$$

Para el caso de la BV solo necesitamos un operador, que esta dado por:

$$
\hat{p}=1 \oplus 0_{B C} \oplus 0_{N_{1}} \oplus 0_{N_{2}},
$$

donde $0_{B C}, 0_{N_{1}}$ y $0_{N_{2}}$ son las matrices nulas de los subsistemas de la $\mathrm{BC}$, de las trampas no apareadas y de las trampas apareadas, respectivamente, por lo tanto $V_{B V}=\{\hat{p}\}$.

El espacio de la BC esta formado por 4 elementos de matriz $\rho_{2,2}, \rho_{2,3}, \rho_{3,2}, \rho_{3,3}$, por lo que necesitamos cuatro operadores. Los generadores de $U(2)$ describen adecuadamente este espacio. Los generadores de este espacio son la matriz identidad y las matrices de Pauli $\left(\hat{\sigma}_{x}, \hat{\sigma}_{y}, \hat{\sigma}_{z}\right)$

$$
U(2)=\left\{\hat{\mathbb{I}}, \frac{1}{2} \sigma_{x}, \frac{1}{2} \sigma_{y}, \frac{1}{2} \sigma_{z}\right\}=\left\{\hat{s}_{0}, \hat{s}_{1}, \hat{s}_{2}, \hat{s}_{3}\right\} .
$$

Los operadores de la $\mathrm{BC}$ en el sistema total esta dado por

$$
\hat{S}_{k}=0_{B V} \oplus \hat{s}_{k} \oplus 0_{N_{1}} \oplus 0_{N_{2}},
$$

donde $0_{B V}$ es la matriz nula de la BV. Este conjunto de generadores forman un grupo de Lie de dimension 4.

$$
\left[\hat{S}_{i}, \hat{S}_{j}\right]=i \hbar \sum_{k} C_{i j}^{k} \hat{S}_{k}
$$

donde $C_{i j}^{k}$ son las constantes de estructura y están definidas como

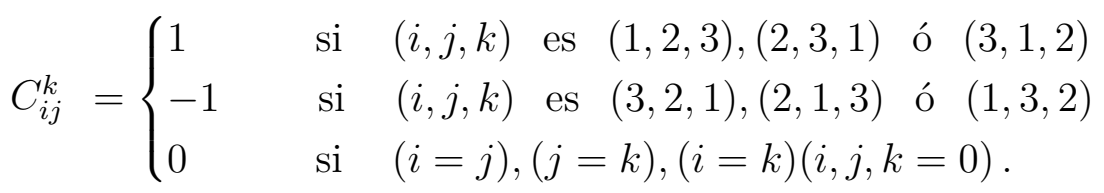

Así que el espacio vectorial de operadores para la BC esta dado por $V_{B C}=$ $\left\{\hat{S}_{k}\right\}$, donde $\mathrm{k}=0,1,2,3$.

En cuanto a las trampas paramagnéticas apareadas, este espacio esta formado por 16 elementos de matriz $\rho_{k, l}$, donde $k=12, \ldots, 15$ y $l=12, \ldots, 15$. Para describir este espacio usamos los 16 generadores de $U(4)$. En este trabajo, obtenemos estos generadores por medio del producto tensorial entre dos grupos $U(2)$

$$
\begin{aligned}
U(2) \otimes U(2) & =\left\{\hat{s}_{j}\right\} \otimes\left\{\hat{s}_{i}\right\} \\
& =\left\{\hat{s}_{0} \otimes \hat{s}_{0}, \hat{s}_{0} \otimes \hat{s}_{1}, \hat{s}_{0} \otimes \hat{s}_{2}, \hat{s}_{0} \otimes \hat{s}_{3}, \ldots\right\} .
\end{aligned}
$$


Para simplificar la notación, representamos al conjunto de operadores que forman el espacio con un tensor de orden- $2\left(f_{j, i}\right)$, donde $j=0, \ldots, 3 \mathrm{e}$ $i=0, \ldots, 3$. Cada elemento del tensor hace alusión a un operador de la base, por ejemplo $\hat{f}_{0,0}=\hat{s}_{0} \otimes \hat{s}_{0}, \hat{f}_{0,1}=\hat{s}_{0} \otimes \hat{s}_{0}, \hat{f}_{1,0}=\hat{s}_{1} \otimes \hat{s}_{0}, \ldots$

Los operadores que describen a las trampas apareadas en el sistema total está dado por

$$
\hat{F}_{j i}=0_{B V} \oplus 0_{B V} \oplus 0_{N_{1}} \oplus \hat{f}_{j i}
$$

por lo que el espacio vectorial de operadores para las trampas apareadas es $V_{N_{2}}=\left\{\hat{F}_{j, i}\right\}$.

Así como los generadores de la $\mathrm{BC}$, los generadores $\hat{F}_{j, i}$ forman un álgebra de Lie de dimension finita. Para este caso la dimensión es 16 y los operadores cumplen con las siguientes reglas de conmutación

$$
\left[\hat{F}_{j i}, \hat{F}_{k l}\right]=i \hbar \sum_{m n} C_{j i k l}^{m n} \hat{F}_{m n}
$$

El espacio de las trampas paramagnéticas no apareadas está formado por 64 elementos de matriz $\rho_{k, l}$, donde $k=4, \ldots, 11$ y $l=4, \ldots, 11$. Para describir este espacio usamos los 64 generadores para $U(8)$. Los generadores provienen del triple producto tensorial entre los generadores de $U(2)$.

$$
\begin{aligned}
U(2) \otimes U(2) \otimes U(2) & =\left\{\hat{s}_{j}\right\} \otimes\left\{\hat{s}_{i}\right\} \otimes\left\{\hat{s}_{i}\right\} \\
& =\left\{\hat{s}_{0} \otimes \hat{s}_{0} \otimes \hat{s}_{0}, \hat{s}_{0} \otimes \hat{s}_{0} \otimes \hat{s}_{1}, \ldots\right\} .
\end{aligned}
$$

Con el fin de abreviar la notación representamos a estos operadores con un tensor de orden-3, donde $u_{k, j, i}, k=0, \ldots, 3, j=0, \ldots, 3$ y $i=0, \ldots, 3$. Cada elemento del tensor hace alusión a un operador de la base, por ejemplo $\hat{u}_{0,0,0}=\hat{s}_{0} \otimes \hat{s}_{0} \otimes \hat{s}_{0}, \hat{u}_{0,0,1}=\hat{s}_{0} \otimes \hat{s}_{0} \otimes \hat{s}_{1}, \hat{u}_{0,0,2}=\hat{s}_{0} \otimes \hat{s}_{0} \otimes \hat{s}_{2}, \ldots$

Los operadores que describen a las trampas no apareadas en el sistema total esta dado por

$$
\hat{U}_{k j i}=0_{B V} \oplus 0_{B V} \oplus \hat{u}_{k j i} \oplus 0_{N_{2}} .
$$

Por lo que el espacio vectorial de operadores para las trampas apareadas es $V_{N_{1}}=\left\{\hat{U}_{k, j, i}\right\}$.

Así como los generadores de la BC y los generadores de las trampas apareadas, los generadores $\hat{U}_{k, j, i}$ forman un grupo de Lie de dimensión 64 , cuyas reglas de conmutación son

$$
\left[\hat{U}_{i j k}, \hat{U}_{l m n}\right]=i \hbar \sum_{m n} C_{i j k l m n}^{p q r} \hat{U}_{p q r}
$$

El espacio vectorial de los 85 operadores del sistema es la suma de los espacios vectoriales $V_{B V}, V_{B C}, V_{N 1}$ y $V_{N 2}$, 


$$
\Lambda=\left\{\hat{p}, \hat{S}_{m}, \hat{U}_{k, j, i}, \hat{F}_{j, i}\right\} .
$$

Así, la matriz de densidad se puede expresar como la combinación lineal

$$
\hat{\rho}_{N}(t)=\sum_{i=1}^{85} \frac{\lambda_{i}(t)}{\operatorname{Tr}\left[\hat{\lambda}_{i} \hat{\lambda}_{i}\right]} \hat{\lambda}_{i},
$$

donde

$$
\lambda_{i}(t)=\operatorname{Tr}\left[\hat{\rho}(t) \hat{\lambda}_{i}\right],
$$

es el valor esperado mecano-estadístico del operador $\hat{\lambda}_{i}$. Así, los valores esperados mecano-estadísticos de los huecos, electrones, trampas apareadas y no apareadas están dados por $p(t)=\operatorname{Tr}[\hat{\rho}(t) \hat{p}], n(t)=\operatorname{Tr}\left[\hat{\rho}(t) \hat{S}_{0}\right]$, $N_{1}(t)=\operatorname{Tr}\left[\hat{\rho}(t) \hat{U}_{0,0,0}\right], N_{2}(t)=\operatorname{Tr}\left[\hat{\rho}(t) \hat{F}_{0,0}\right], \ldots$

\subsection{Hamiltoniano}

El Hamiltoniano del sistema es:

$$
\hat{H}=\boldsymbol{\omega} \cdot \hat{\boldsymbol{S}}+\boldsymbol{\omega}_{c} \cdot \hat{\boldsymbol{S}}_{c}+\mathcal{A} \hat{\boldsymbol{S}}_{c} \cdot \hat{\boldsymbol{I}}_{1}
$$

donde $\hat{\boldsymbol{S}}$ y $\hat{\boldsymbol{S}}_{c}$ son los operadores de espín para los electrones en la banda de conducción y en las trampas paramagnéticas, respesctivamente; $\hat{\boldsymbol{I}}_{1}$ es el operador de espín nuclear de la trampa no apareada del $\mathrm{Ga}^{+2} \cdot \boldsymbol{\omega}=g \mu_{B} \boldsymbol{B} / \hbar$, $\boldsymbol{\omega}_{c}=g_{c} \mu_{B} \boldsymbol{B} / \hbar, \mu_{B}$ es el magnetón de Bhor, $\boldsymbol{B}$ es el campo magnético externo, $g=+1$ y $g_{c}=+2$ son los factores giromagnéticos de los electrones en la $\mathrm{BC}$ y en las trampas [5, 73, 74]. $\mathcal{A}$ es la constante hiperfina. Los isótopos que más abundan en el GaAsN son el Ga ${ }^{69}$ con $\mathcal{A}=620 \times 10^{-4} \mathrm{~cm}^{-1}$ y el $\mathrm{Ga}^{71} \mathcal{A}=788 \times 10^{-4} \mathrm{~cm}^{-1}$ con porcentajes del $60 \%$ y $40 \%$, respectivamente. Tomamos el promedio ponderado entre los dos dando un valor de $\mathcal{A}=704 \times 10^{-4} \mathrm{~cm}^{-1}$. Los primeros dos términos de la ecuación (3.18) son los debidos a la interacción Zeeman entre el espín de los electrones de la $\mathrm{BC}$ y de las trampas con un campo magnético externo. El último término corresponde al acoplamiento hiperfino entre el espín del electrón ligado al centro paramagnético no apareado con su espín nuclear. Los operadores que forman el hamiltoniano están definidos en el apéndice A.

\subsection{Disipador}

El principal problema al resolver un sistema cuántico abierto es la determinación del disipador. En el disipador se encuentran las interacciones entre 
el sistema y sus alrededores [1-3], 75]. Éste fué elegido de tal manera que reprodujera las ecuaciones del modelo de dos cargas [4, 15, 76] y que fuera invariante ante rotaciones. El disipador es

$$
\boldsymbol{D}(\hat{\boldsymbol{\rho}})=\mathcal{D}_{S D R}+\mathcal{D}_{S}+\mathcal{D}_{S_{c}}+\mathcal{D}_{1}+\mathcal{D}_{2}+\mathcal{G}
$$

donde $\mathcal{D}_{S D R}$ contiene los mecanismos de la SDR descritos por el [TCM. $\mathcal{D}_{S}$ y $\mathcal{D}_{S_{c}}$ describen las perdidas de espín electrónico en la $\mathrm{BC}$ y en las trampas no apareadas, respectivamente, debido a los tiempos de relajación $\tau_{s}$ y $\tau_{s c}$. $\mathcal{D}_{1}$ y $\mathcal{D}_{2}$ dan cuenta de las perdidas de espín nuclear en las trampas no apareadas $\left(N_{1}\right)$ y apareadas $\left(N_{2}\right)$. Por último, el termino $\mathcal{G}$ modela la generación de electrón-hueco en la BC y en la BV] así mismo modela la inyección del espín en la BC.

El disipador del mecanismo de la SDR esta dado por

$$
\mathcal{D}_{S D R}=\sum_{q=1}^{85} \frac{C\left[\lambda_{q}\right]}{\operatorname{Tr}\left[\lambda_{q}^{2}\right]} \hat{\lambda}_{q}
$$

donde los coeficientes $C\left[\lambda_{q}\right]=\operatorname{Tr}\left[\mathcal{D}_{S D R} \hat{\lambda}_{q}\right]$ son en principio desconocidos. Sin embargo para darnos una idea de la forma explicita de estos coeficientes, comparamos las ecuaciones cinéticas que surgen de (3.1) cuando $H=0 \mathrm{y}$ $\mathcal{D}=\mathcal{D}_{S D R}$ con el modelo de dos cargas 2.5 - 2.10). Multiplicando a (3.1) por $\hat{\lambda}_{q}$ que es el elemento q-ésimo de los operadores que forman el espacio (3.15), y tomamos la traza, tenemos que

$$
\frac{d \lambda_{q}}{d t}=C\left[\lambda_{q}\right]
$$

con esta relación podemos inferir que los coeficientes $C\left[\lambda_{q}\right]$ están dados por los téminos del lado derecho de las ecuaciones de balance del TCM (2.5)(2.10). Los coeficientes están dados por 


$$
\begin{aligned}
C[p] & =-\gamma_{r} n p-\gamma_{h} p F_{0,0}+G, \\
C[n] & =-\gamma_{r} n p-\frac{\gamma_{e}}{2}\left(n U_{0,0,0}-4 \sum_{k=1}^{3} S_{k}^{n} U_{k, 0,0}\right)+G, \\
C\left[S_{k}\right] & =-\gamma_{r} S_{k} p+\frac{\gamma_{e}}{2}\left(n U_{r, 0,0}-S_{r}^{n} U_{0,0,0}\right)+G_{s}, \\
C\left[U_{0, j, i}\right] & =-\frac{\gamma_{e}}{2}\left(n U_{0, j, i}-4 \sum_{r=1}^{3} S_{r}^{n} U_{r, j, i}\right)+\gamma_{h} p F_{j, i}, \\
C\left[U_{k, j, i}\right] & =-\frac{\gamma_{e}}{2}\left(n U_{k, j, i}-S_{k}^{n} U_{0, j, i}\right) \\
C\left[F_{j, i}\right] & =\frac{\gamma_{e}}{2}\left(n U_{0, j, i}-4 \sum_{r=1}^{3} S_{r}^{n} U_{r, j, i}\right)-\gamma_{h} p F_{j, i},
\end{aligned}
$$

donde $k=1,2,3$ e $i, j=0,1,2,3$. Además $\gamma_{e}=1 / N_{c} \tau^{*}$ y $\gamma_{h}=1 / N_{c} \tau_{h}$ son las razones de recombinación para los electrones en la BC y los huecos en la BV] donde $N_{c}=3 \times 10^{15} \mathrm{~cm}^{-3}$ es el número total de centros $\mathrm{Ga}^{2+}$ [74, 77] y $\tau^{*}=4.4$ ps y $\tau_{h}=12$ ps son los tiempos de recombinación de los electrones de la BC y de los huecos en la BV [1].

Para modelar las pérdidas de espín electrónico en la banda de conducción $\left(\mathcal{D}_{S}\right)$ y en las trampas no apareadas $\mathcal{D}_{S_{c}}$ usamos la teoría de relajación de Wangness-Bloch-Redfield [1, 78 [81]. Los disipadores están dados por

$$
\begin{aligned}
\mathcal{D}_{S} & =-\frac{1}{2 \tau_{s}} \sum_{k=1}^{3}\left[\hat{S}_{k},\left[\hat{S}_{k}, \hat{\rho}\right]\right], \\
\mathcal{D}_{S C} & =-\frac{1}{2 \tau_{s c}} \sum_{k=1}^{3}\left[\hat{S}_{c, k},\left[\hat{S}_{c, k}, \hat{\rho}\right]\right],
\end{aligned}
$$

donde los tiempos de relajación $\tau_{s}=110$ ps y $\tau_{s c}=1700$ ps [1]. $\hat{S}_{k}$ y $\hat{S}_{c, k}$ son los operadores de espín en el espacio total para los electrones en la BC y en las trampas no apareadas, respectivamente.

También usamos la teoría de relajación de Wangness-Bloch-Redfield para modelar las pérdidas de espín nuclear de las trampas no apareadas $\mathcal{D}_{1}$ y apareadas $\mathcal{D}_{2}$. Para esto consideramos que hay una interacción dipolo-dipolo entre el espín nuclear de las trampas con los espines nucleares de los Galios vecinos que forman la red. 


$$
\begin{aligned}
& \mathcal{D}_{1}=-\frac{1}{2 \tau_{n 1}} \sum_{k=1}^{3}\left[\hat{I}_{1, k},\left[\hat{I}_{1, k}, \hat{\rho}\right]\right], \\
& \mathcal{D}_{2}=-\frac{1}{2 \tau_{n 2}} \sum_{k=1}^{3}\left[\hat{I}_{2, k},\left[\hat{I}_{2, k}, \hat{\rho}\right]\right],
\end{aligned}
$$

donde los tiempos de relajación de espín nuclear dados por $\tau_{n 1}=5405$ ps y $\tau_{n 2}=446 \mathrm{ps}$ fueron estimados de trabajos previos [1]. Los operadores $\hat{I}_{1} \mathrm{y}$ $\hat{I}_{2}$ son los operadores de espín nuclear en el espacio total para los núcleos de las trampas no apareadas y apareadas, respectivamente.

Para finalizar, modelamos el término de la inyección de espín en la $\mathrm{BC}$ y la generación de electrón-hueco en la BC y BV] de la siguiente manera

$$
\mathcal{G}=(n+p)\left(G_{+}+G_{-}\right)+\left(G_{+}-G_{-}\right) \boldsymbol{e} \cdot \boldsymbol{S},
$$

donde el primer término de la ecuación anterior es el término de generación de electrón-hueco y el segundo término esta relacionado con la generación de electrones polarizados en la dirección del vector unitario $\boldsymbol{e}$, en otras palabras, está relacionado con la inyección de espín en la banda de conducción. $G_{+}$y $G_{-}$son funciones del tiempo que describen la fotogeneración de electrones con espín hacia arriba y con espín hacia bajo la incidencia de un haz de luz.

Para este trabajo, consideramos que el pulso del haz incidente tiene un perfil gaussiano

$$
G(t)_{ \pm}=\frac{G_{0} P t_{p p}}{2 \sqrt{2 \pi}} \frac{1 \pm P_{e}}{s_{t}} e^{-\frac{1}{2}\left(\frac{t-t_{0}}{s_{t}}\right)^{2}}
$$

donde P es la potencia del haz, $G_{0}=3.03 \times 10^{23} \mathrm{~cm}^{-3}$ es la cantidad promedio de fotoelectrones generados. El signo positivo indica que el haz es circularmente polarizado a la izquierda y el signo menos que es circularmente polarizado a la derecha. El ancho del pulso es $s_{t}=1.5 \mathrm{ps}, t_{0}$ es el tiempo de encendido, $t_{p p}=10 n$ s es el tiempo de duración del pulso y $P_{e}$ es el porcentaje de polarización del haz. Para el caso de luz linealmente polarizada $\left|G_{+}-G_{-}\right|=0$ y para luz circularmente polarizada $\left|G_{+}-G_{-}\right|=0.5$ 


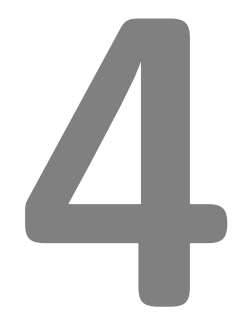

\section{Resultados}

U

samos el modelo desarrollado en el capítulo anterior para analizar la dinámica del espín de los electrones de la [BC, de las trampas paramagnéticas y del espín nuclear de las trampas en régimen pulsado, con el fin de trazar y detectar el flip-flop de los centros paramagnéticos en el GaAsN mediante bombeo óptico en régimen pulsado.

Para cumplir con nuestro cometido, primero analizamos al sistema con un solo tipo $\mathrm{Ga}^{2+}$ en presencia de un pulso, después estudiamos al sistema bajo la incidencia de dos pulsos consecutivos. A partir de los resultados obtenidos con dos pulsos consecutivos propusimos un esquema pulso-prueba para detectar y trazar el flip-flop de los centros paramagnéticos del GaAsN en régimen pulsado [1]. Después mostramos los resultados teóricos y experimentales para un sistema de mayor complejidad el de dos isótopos de $\mathrm{Ga}^{2+}[2]$. Para finalizar, presentamos los resultados del modelo en régimen pulsado en presencia de un campo magnético oblicuo a la dirección de polarización. Mostramos diferentes pruebas para varios valores de la intensidad de la potencia del pulso-prueba y diferentes ángulos de inclinación del campo magnético, con el proposito de encontrar los valores óptimos para detectar y trazar el flip-flop de los centros en las tres componentes espaciales de la polarización del espín.

Con el fin de extraer información del modelo, comenzamos construyendo el sistema de ecuaciones que provinen de la ecuación maestra (3.1). Introducimos las expresiones del Hamiltoniano (3.18), la matriz de densidad (3.16) y el disipador (3.19) en la ecuación maestra (3.1). Multiplicamos de los dos lados de la ecuación (3.1) por $\hat{\lambda}_{q}$ y tomamos la traza de los dos lados de la ecuación. Obtenemos un sistema de 85 ecuaciones diferenciales no lineales de primer orden

$$
\operatorname{Tr}\left[\hat{\lambda}_{q} \frac{d \hat{\rho}}{d t}\right]=\frac{d \lambda_{q}(t)}{d t}=\operatorname{Tr}\left[\frac{1}{i \hbar}[\hat{H}, \hat{\rho}] \hat{\lambda}_{q}+\hat{\mathcal{D}} \hat{\lambda}_{q}\right], q=1,2, . ., 85
$$


donde $\hat{\lambda}_{q}$ es el operador q-esimo de la base (3.15) y $\lambda_{q}(t)$ su valor mecanoestadístico. Cabe mencionar que este sistema considera los mecanismos de recombinación dependiente del espín, la interacción hiperfina y la interacción Zeeman. Además, las perdidas de espín electrónico en la BC y en las trampas paramagnéticas no apareadas; las pérdidas de espín nuclear de las trampas apareadas y no apareadas.

Estudiamos la dinámica del espín de los electrones y de los núcleos con el sistema de ecuaciones (4.1). Construimos y resolvimos númericamente dicho sistema con un algoritmo hecho en Mathematica [82]. El sistema de 85 ecuaciones diferenciales no lineales de primer orden solo tiene 8 parámetros libres que son: $\gamma_{r}=0.05$ la razón de recombinación bimolecular entre la BC y la BV. $\gamma_{e}=1 / N_{c} \tau^{*}$ y $\gamma_{h}=1 / N_{c} \tau_{h}$ son las razones de recombinación para los electrones en la BC y los huecos en la BV, donde $N_{c}=3 \times 10^{15} \mathrm{~cm}^{-3}$ es el número total de centros $\mathrm{Ga}^{2+}$ [74, 77] y $\tau^{*}=4.4$ ps y $\tau_{h}=12 \mathrm{ps}$ son los tiempos de recombinación de los electrones de la BC y de los huecos en la BV. $\tau_{s}=110$ ps y $\tau_{s c}=1700$ ps son los tiempos de relajación del espín para los electrones de la BC y de las trampas no apareadas. $\tau_{n 1}=5405$ ps y $\tau_{n 2}=446$ ps son los tiempos de relajación de espín nuclear para las trampas no apareadas y para las trampas apareadas, respectivamente [1].

Los parámetros relevantes son los promedios mecano-estadísticos $\lambda_{q}(t)$ de los operadores $\hat{\lambda}_{q}$. Asumimos que antes de la excitación óptica en $t=0$ las trampas no apareadas son igualmente probables y que los electrones y los núcleos están completamente despolarizados. Por lo tanto, $N_{1}(0)=\lambda_{6}(0)=$ $N_{c}$ y $\lambda_{q}(0)=0$ para $q \neq 6$. Notamos que esta condición inicial también implica que en este estado no hay trampas apareadas por lo que $N_{2}(0)=$ $\lambda_{70}(0)=0$.

Las principales cantidades experimentales calculadas son la fotoluminiscencia $(I(t))$, las polarizaciones electrónicas en la BC $\left(S_{z}(t)\right)$ y en las trampas no apareadas $\left(S_{c z}(t)\right)$; además de las polarizaciones nucleares de las trampas no apareadas $\left(I_{1 z}(t)\right)$ y apareadas $\left(I_{2 z}(t)\right)$.

La fotoluminiscencia o intensidad de la radiación de fotoluminiscencia entre la BC y la BV esta dada por la razón de recombinación bimolecular

$$
I(t)=\gamma_{r} n(t) p(t)
$$

donde $\gamma_{r}$ es la razón de recombinación bimolecular, $n(t)$ y $p(t)$ son los valores de expectación de los operadores de los electrones en la BC y de los huecos en la $\mathrm{BV}$, respectivamente, en nuestra notación $p(t)=\lambda_{1}(t)$ y $n(t)=\lambda_{2}(t)$. Así mismo, determinamos la fotoluminiscencia para los casos en que tenemos luz incidente circularmente polarizada a la izquierda $\left[I_{-}(t)\right.$ y linealmente polarizada $I_{x}(t)$. Con estos valores calculamos el cociente entre ellas, que 
denominamos razón de recombinación dependiente del espín

$$
S D R_{r}(t)=100 \frac{I_{-}(t)}{I_{x}(t)}
$$

Dado que bajo la incidencia de luz circularmente polarizada la densidad de electrones fotogenerados en la BC tienen polarización de espín promedio diferente de cero, la intensidad de la fotoluminiscencia bajo luz circularmente polarizada es mayor que bajo la incidencia de luz linealmente polarizada. De esta forma $S D R_{r}$ es un parámetro apropiado para medir la presencia del fenómeno de la SDR. Entonces, cuando las trampas se polarizan y bloquean la recombinación de electrones a través de ellas, la población de electrones en la BC aumenta incrementando también la intensidad de la fotoluminiscencia, en este caso $S D R_{r}>100$. Por el contrario cuando $S D R_{r}=100$ la polarización de espín en la BC es nula.

Por último determinamos los valores mecano-estadísticos de las polarizaciones de espín electrónico para la banda de conducción $(\vec{S}(t))$ y para las trampas no apareadas $\left(\vec{S}_{c}(t)\right)$; las polarizaciones de espín nuclear para las trampas no apareadas $\left(\vec{I}_{1}(t)\right)$ y para las trampas apareadas $\left(\vec{I}_{2}(t)\right)$.

En las dos siguientes secciones presentamos los resultados para los casos en ausencia y presencia de un campo magnético externo cuando hay un solo isótopo y en la presencia de dos isótopos. 

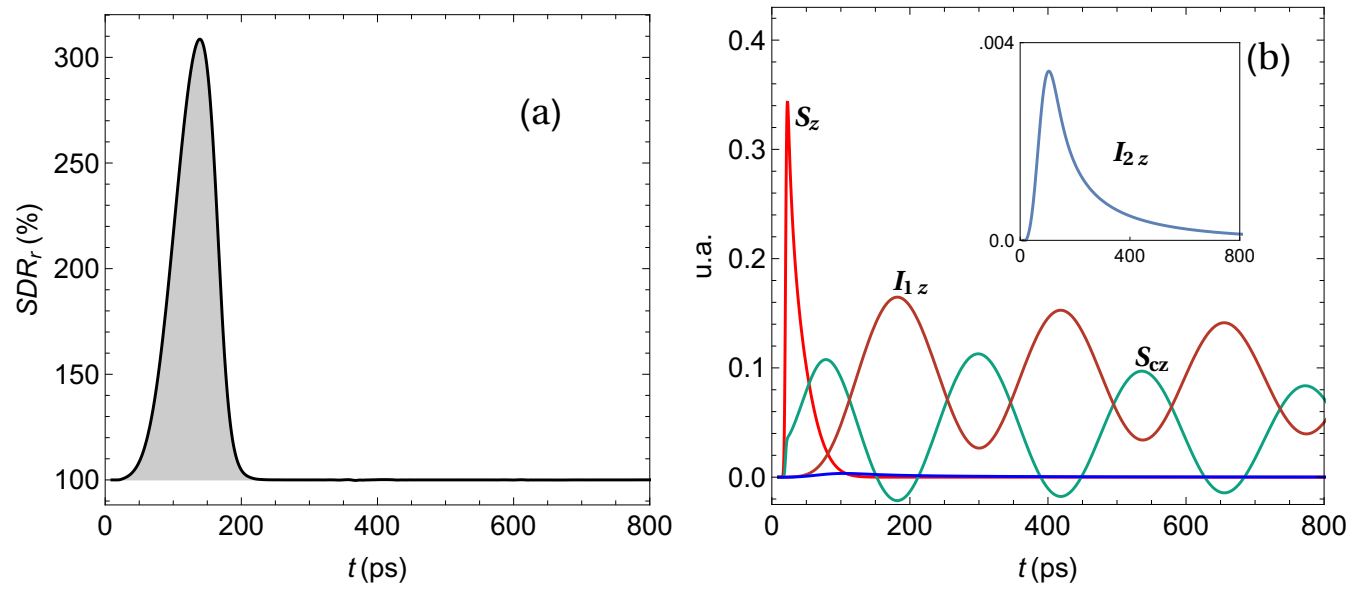

Figura 4.1: En la Figura (a) mostramos la $S D R_{r}$ en función del tiempo en ausencia de campo magnético. Notamos que el $S D R_{r}$ tiene un máximo al pulso lanzado. En la Figura (b), presentamos las polarizaciones de espín electrónica de la BC $\left(S_{z}\right.$ curva roja), de espín electrónica de las trampas no apareadas $\left(S_{c z}\right.$ curva café), de espín nuclear de las trampas no apareadas $I_{1 z}$ (curva verde) y de espín nuclear de las trampas apareadas $I_{2 z}$ (curva azul) en ausencia de un campo magnético. Las polarizaciones $I_{2 z}$ y $S_{z}$ crecen hasta un tiempo máximo y depués decrecen monotonamente hasta cero. Las curvas $I_{1 z}$ y $S_{c z}$ tienen un comportamiento sinosoidal evanescente. El comportamiento oscilante se le denomina flip-flop el cual es debido a la interacción hiperfina entre los espines nuclear y electrónico de la trampa no apareada. El comportamiento evanescente se debe a las perdidas de espín electrónico y nuclear

\subsection{Resultados para un isótopo}

En esta sección mostramos los resultados obtenidos con el modelo considerando que las trampas paragamnéticas en el GaAsN solo están formadas por un isótopo promedio de $\mathrm{Ga}^{2+}$.

Comenzamos nuestra discusión con el comportamiento de la $S D R_{r}(t)$ como función del tiempo ante un pulso con $P=60 \mathrm{~mW}$ y una duración de $s_{t}=1.5$ ps sobre la muestra GaAsN.

En la Figura 4.1 (a), notamos que la $S D R_{r}$ aumenta conforme incrementa el tiempo, hasta un tiempo crítico $\left(t_{c}\right)$, donde la $S D R_{r}$ es máxima. Para este caso, se tiene que $t_{c}=120$ ps con un valor de $S D R_{r}(t)=309$. Para tiempos mayores a $t_{c}$ la $S D R_{r}$ disminuye monótonamente hasta 100 a un tiempo de 220 ps. Dichos resultados muestran que hay un aumento de la fotoluminiscencia al incidir luz circularmente polarizada en aleaciones de GaAsN a temperatura ambiente ([4] [5]).

En la figura 4.1 (b) exhibimos el comportamiento de las polarizaciones del espín de los electrones en la $\mathrm{BC}\left(S_{z}\right)$, del espìn de los electrones de las trampas no apareadas $\left(S_{c z}\right)$, del espín nuclear de las trampas no apareadas $\left(I_{1 z}\right)$ y del 

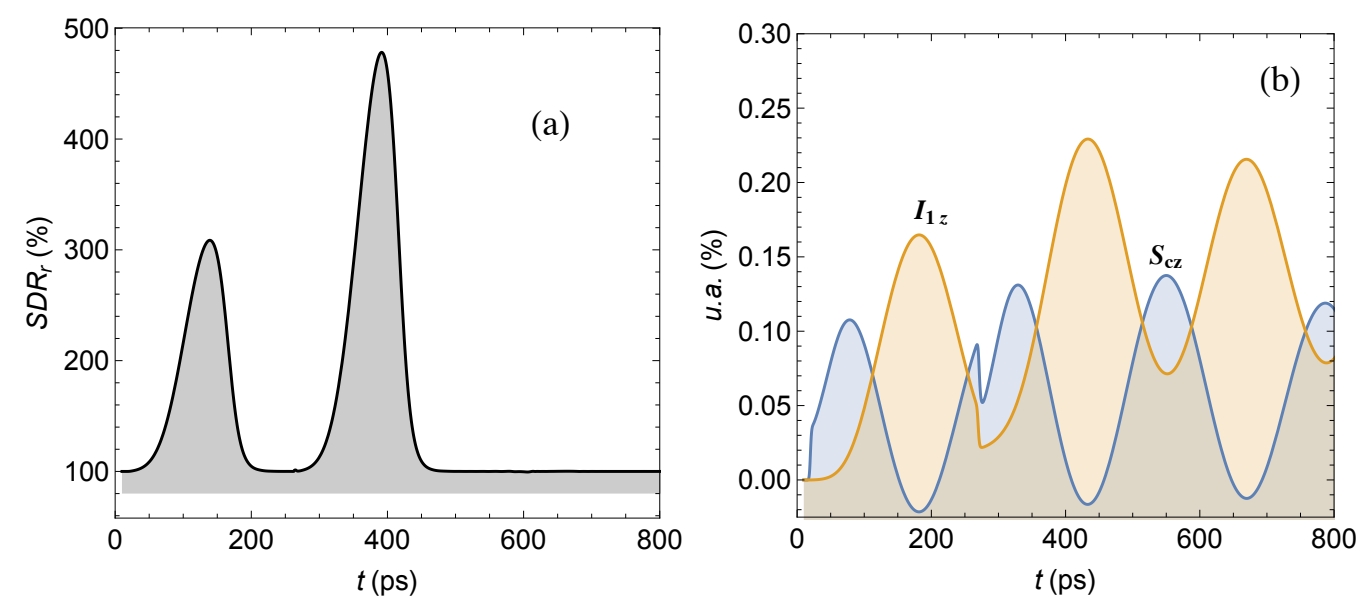

Figura 4.2: En la Figura (a) mostramos la $S D R_{r}$ en función del tiempo en ausencia de un campo magnético externo en presencia de dos pulsos consecutivos. Notamos que el $S D R_{r}$ tiene dos máximos, esto se debe a la presencia de los dos pulsos. Observamos que el máximo del segundo pulso es más grande que el primero. Este aumento en el $S D R_{r}$ se debe a la información remanente del primer pulso. En la Figura (b), mostramos las curvas de polarización electrónica ( $S_{c z}$ curva azul) y nuclear ( $I_{1 z}$ curva naranja) de las trampas no apareadas. Las curvas de polarización muestran el flip-flop de espín electrón-núcleo causado por la interacción hiperfina en las trampas no apareadas. Dichas curvas tienen un cambio abrupto en $t=270$ ps debido a la incidencia del segundo pulso. Notamos que este aumento en las polarizaciones Figura (b) se ve reflejado en el aumento del segundo pico

espín nuclear de los centros apareados $\left(I_{2 z}\right)$. En primer lugar, observamos que la curva de la polarización de espín en la BC crece rápidamente en el tiempo; alcanza su valor máximo en $t=12$ ps con un valor de $S_{z}=0.34$, después la polarización decrece rápidamente. En segundo lugar, nos percatamos que la polarización de espín nuclear para las trampas apareadas (linea azul) crece lenta y monótonamente en el tiempo hasta $t=125$ ps donde alcanza su valor máximo con $I_{2 z}=.0035$, posteriormente decrece lentamente.

Notamos que las curvas correspondientes a las polarizaciones de espín electrónico y nuclear de las trampas no apareadas tienen un comportamiento oscilatorio. Observamos que el mínimo de $I_{1 z}$ (curva café) corresponde al máximo valor de $S_{c z}$ (curva verde). Al comportamiento de estas dos polarizaciones se le denomina proceso de flip-flop, dicho efecto se debe a la interacción hiperfina que hay entre el espín electrónico y nuclear de la trampa no apareada. También notamos que la magnitud de los mínimos y máximos de las polarizaciones cambian en el tiempo. El cambio de los valores máximales de las polarizaciones se deben a los términos de relajación que incluimos para el espín electrónico y el espín nuclear de la trampa no apareada. Estos términos de relajación están asociados con $\tau_{s c}$ y $\tau_{n_{1}}$ respectivamente. 
Continuamos la discusión sobre el comportamiento de la $S D R_{r}(t)$ cuando están presentes dos pulsos consecutivos en ausencia de campo magnético. Los pulsos tienen la misma intensidad y la misma duración pero son introducidos de manera consecutiva. Encendemos el primero en $t=20 \mathrm{ps}$ y el segundo en $t=270$ ps. El segundo tiempo 270 ps se elegió por dos razones. La primera razón se debe a que en ese tiempo la polarización electrónica en la $\mathrm{BC}$ es prácticamente cero como puede verse en la figura 4.1 (b). La segunda es porque para $t=270 \mathrm{ps}$, las polarizaciones de espín electrónico y nuclear se encuentran cerca de sus valores máximo y mínimo, respectivamente. Este segundo pulso tiene el fin de mostrar que hay una contribución remanente del primer pulso através de la $S D R_{r}$. Cabe destacar que aunque ya no hay polarización de espín en la $\mathrm{BC}$, el flip-flop de la polarización electrónica y nuclear de la trampa no apareada se mantiene.

En la figura 4.2 (a) observamos que la $S D R_{r}(t)$ tiene dos máximos en $t=123$ y $t=400 \mathrm{ps}$, con valores de $S D R_{r} 309$ y 490, respectivamente. Este comportamiento se debe a la incidencia de los dos pulsos consecutivos. Aunque el segundo pulso fue lanzado en un tiempo en el que la polarización en la BC era nula, el segundo pico es más alto que el primero. Esto quiere decir que hay un aumento en la polarización de los electrones en la BC.

Notamos que las curvas correspondientes a las polarizaciones del espín electrónico y del espín nuclear de las trampas no apareadas tienen el mismo comportamiento sinusoidal que para el caso de un solo pulso Figura 4.2 (b). Sin embargo, en este caso las dos curvas de polarización cambian abruptamente en $t_{2}=270 \mathrm{ps}$. En dicho tiempo se superpone una sinusoidal en cada polarización. Las magnitudes de los máximos y mínimos de las polarizaciones cambian en el tiempo antes y después de $t_{2}$

La superposición de las dos funciones periódicas para las dos polarizaciones se debe a la presencia del segundo pulso. Notamos que las polarizaciones electrónica y nuclear de la trampa no apareada generadas por el primer pulso a $t_{2}=400$ ps no son cero, por lo que dicha superposición es consecuencia de que en el sistema quedó guardada la información de la polarización generada por del primer pulso.

Debido al comportamiento mostrado por la $S D R_{r}$ y las polarizaciones para dos pulsos Figura 4.2 creemos que por medio de un experimento de pulso-prueba y midiendo la fotoluminiscencia podemos ver la influencia directa del flip-flop entre el espín electrónico y nuclear de la trampa en la polarización de los electrones en la $\mathrm{BC}$. El experimento propuesto consiste en los siguientes pasos.

UAM-Iztapalapa

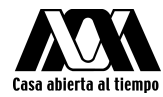




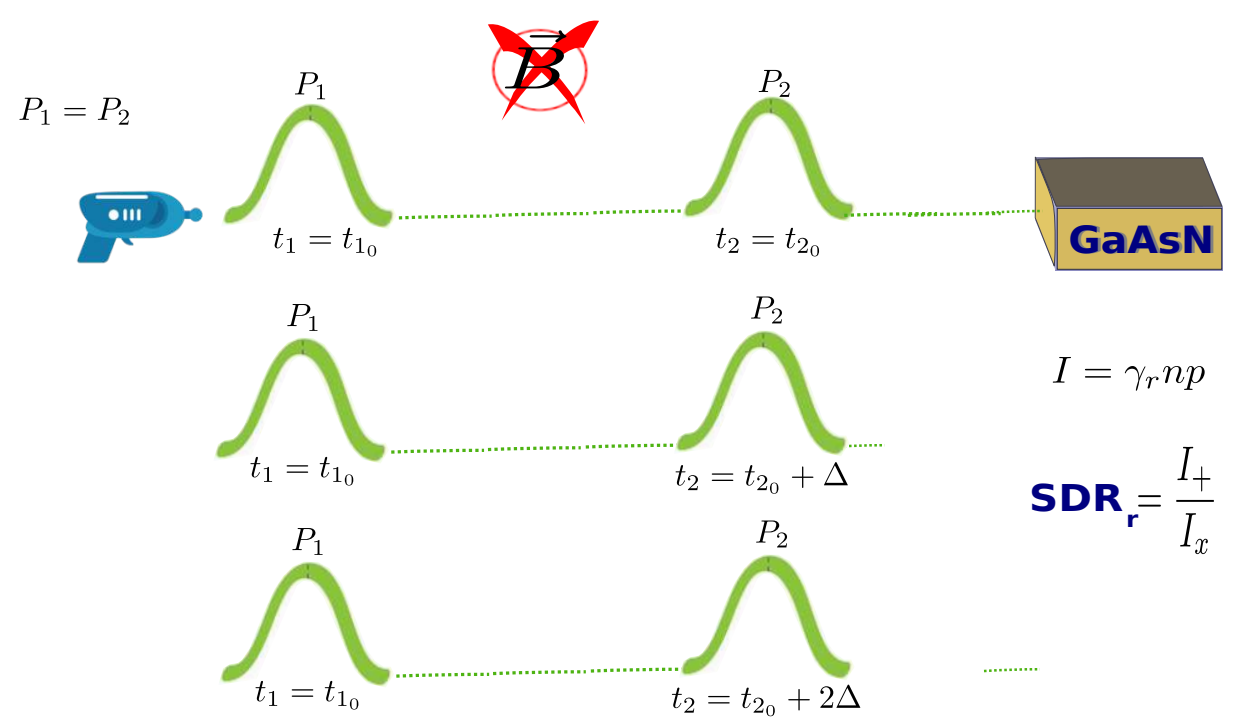

Figura 4.3: Bosquejo de la propuesta del experimento. La propuesta del experimento se centra en realizar varias pruebas donde en cada prueba incidimos dos pulsos consecutivos con la misma intensidad en ausencia de un campo magnético externo para los casos de luz circularmente polarizada y luz linealmente polarizada con el fin de medir $S D R_{r}=I_{-} / I_{x}$ en cada prueba. Para cada situación se mantiene fijo el tiempo de incidencia del primer pulso $\left(t_{10}\right)$ y se aumenta el tiempo de incidencia del segundo pulso en una proporición de $t_{2}=t_{20}+\Delta t$.

- Incidimos dos pulsos consecutivos con luz linealmente polarizada con tiempos de encendido en $t_{10}$ y $t_{20}$. Después medimos la fotoluminiscencia $\left(I_{X}\right)$.

- Incidimos dos pulsos consecutivos con luz circularmente polarizada a la derecha con tiempos de encendido $t_{10}$ y $t_{20}$. A continuación medimos la fotoluminiscencia $\left(I_{+}\right)$.

- Calculamos la $S D R_{r}=\frac{I_{+}}{I_{X}}$.

- Volvemos a realizar los tres pasos anteriores manteniendo fijo el tiempo de encendido del primer pulso y aumentamos el tiempo de encendido del segundo pulso en la proporción de $t_{2}=t_{20}+\Delta t$.

Los pulsos deben de tener la misma intensidad y el experimento debe de realizarse en ausencia de un campo magnético externo. La propuesta puede ser hecha para luz circularmente polarizada a la izquierda $\left(\sigma_{+}\right)$y a la derecha $\left(\sigma_{-}\right)$. En la Figura 4.3 esquematizamos la propuesta del experimento. 
En ésta, modelamos los pulsos de la siguiente manera

$$
G(t)_{ \pm}=\frac{G_{0} P t_{p p}}{2 \sqrt{2 \pi} s_{t}}\left[\left(1 \pm P_{e 1}\right) \mathrm{e}^{-\frac{1}{2}\left(\frac{t-t_{10}}{s_{t}}\right)^{2}}+\left(1 \pm P_{e 2}\right) \mathrm{e}^{-\frac{1}{2}\left(\frac{t-\left(t_{20}+\Delta t\right)}{s_{t}}\right)^{2}}\right]
$$

donde $P$ es la potencia del haz, $G_{0}=3 \times 10^{23} \mathrm{~cm}^{-3} \mathrm{~mW}^{-1}$ es la cantidad promedio de fotoelectrones generados, el signo positivo indica que el haz está circularmente polarizado a la izquierda y el signo negativo que está circularmente polarizado a la derecha. El ancho del pulso es $s_{t}, t_{10}$ y $t_{20}$ son los tiempos de en encendido del primero pulso y del pulso prueba, $t_{p p}=10$ ns es el tiempo de promediación. $\Delta t$ es el tiempo de retardo entre el primer y segundo pulso. El grado de polarización del primer pulso y del pulso prueba están parametrizados por $P_{e 1}, P_{e 2} \in[-1,1]$, respectivamente.

Prosiguiendo con nuestro análisis de fotoluminiscencia, en la Figura 4.4 (a) mostramos la respuesta del sistema ante la aplicación de la propuesta experimental [1]. Cada punto representa el máximo $S D R_{r}(\Delta t)$ del pulso prueba como función del tiempo de retraso $\Delta t$. Los dos pulsos tienen la misma intensidad y la misma desviación estandar $s_{t}=1.5 \mathrm{ps}$. Nos percatamos que los puntos de la $S D R_{r}(\Delta t)$ forman una curva sinusoidal, donde los máximos y mínimos de la curva decrecen conforme aumenta el tiempo. La frecuencia de oscilación es $\omega_{I H F} \approx 4.22 \mathrm{GHz}$. Dicha frecuencia corresponde a la energía de la constante hiperfina $\mathcal{A}=704 \times 10^{-4} \mathrm{~cm}^{-1}$ del isótopo promedio.

El comportamiento sinusoidal de la $S D R_{r}(\Delta t)$ del pulso prueba se debe a la influencia en la fotoluminiscencia del flip-flop entre el espín electrónico y nuclear de las trampas no apareadas mediada por la interacción hiperfina. Es decir, que el flip-flop de los centros paramagnéticos esta relacionado fuertemente con la polarización de los electrones de la BC. La variación de los máximos y mínimos se debe a la relajación del espín nuclear y del espín electrónico de las trampas. Por lo que el experimento de pulso prueba es capaz de detectar y trazar el flip-flop de los centros paramagnéticos del GaAsN mediante bombeo óptico en régimen pulsado en ausencia de un campo magnético externo.

Más específicamente, las oscilaciones del espín electrónico del $\mathrm{Ga}^{2+}$ producen que la eficiencia del filtrado de espín fluctúe y esto se ve reflejado en la fotoluminiscencia. Con el fin de explicar estas fluctuaciones consideraremos dos situaciones extremas en la polarización electrónica de las trampas. En la figura 4.4 (b), mostramos las polarizaciones de espín electrónica (línea azul) y nuclear (línea punteada) de las trampas debido al primer pulso $\left(P_{0}\right)$. En la primera situación, incidimos el pulso-prueba en $t_{p 1}=300$ ps donde la polarización electrónica de las trampas debido al primer pulso es máxima. En este primer escenario, los fotoelectrones generados que tengan la dirección de 

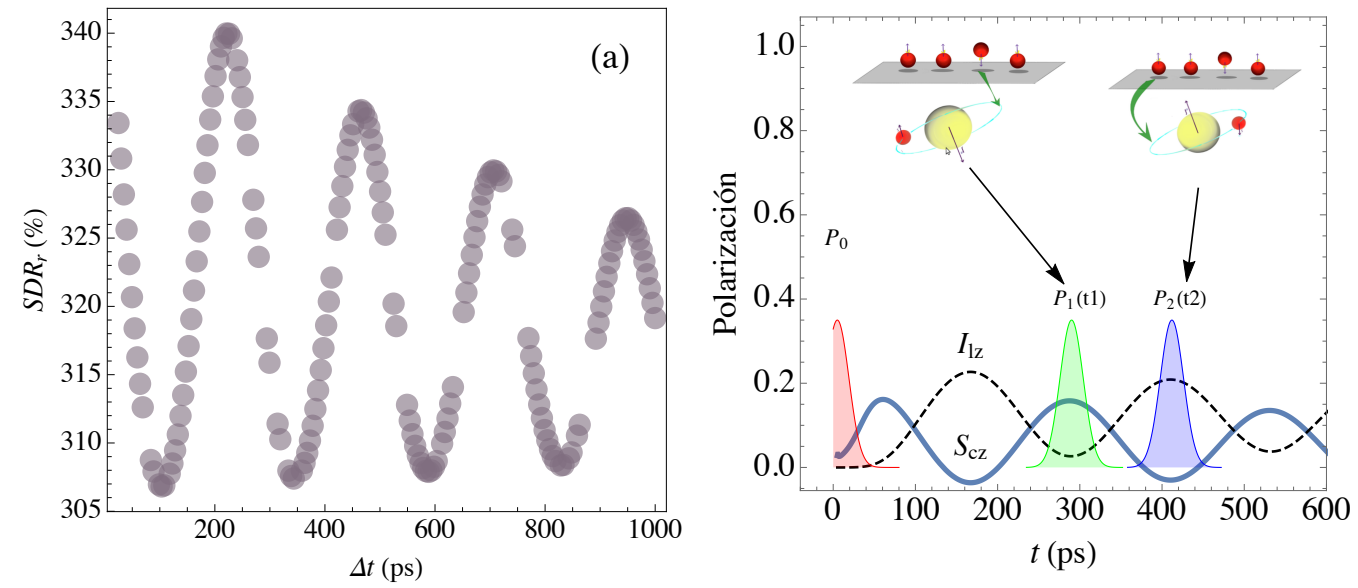

Figura 4.4: En la Figura (a), graficamos los máximos del $S D R_{r}(\Delta t)$ como función del tiempo entre el primer pulso y el segundo $(\Delta t)$ en ausencia de campo magnético. Notamos que los máximos de $S D R_{r}(\Delta t)$ muestran el flip-flop de espín electrón-núcleo de los centros paramagnéticos, la frecuencia de oscilación es $\omega_{I H F} \approx 23.7 \mathrm{GHz}$. Dicha frecuencia corresponde a la energía de la constante hiperfina $\mathcal{A}=704 \times 10^{-4} \mathrm{~cm}^{-1}$. Con el fin de explicar las fluctuaciones en el filtrado de espín, en la Figura (b) mostramos dos situaciones extremas en la polarización electrónica de las trampas. Las curvas azul y punteada representan las polarizaciones electrónica y nuclear, respectivamente, de las trampas no apareadas debido al primer pulso $\left(P_{0}\right)$. En la primera situación, incidimos un pulso-prueba $\left(P_{1}\right)$ cuando la polarización electrónica de las trampas es máxima y en la segunda situación incidimos un pulso-prueba $\left(P_{2}\right)$ cuando la polarización es mínima. La máxima polarización significa que los electrones en las trampas están alineados en la misma dirección que excitamos inicialmente. En cambio, cuando la polarización es mínima los electrones de las trampas están polarizados en la dirección opuesta. Los pulsos $P_{0}, P_{p 1}, P_{p 2}$ inyectan espín en la misma dirección. En la primera situación el filtrado es máximo ya que los pocos electrones con polarización de espín opuesta a la dirección de inyección inicial se recombinan rápidamente en las trampas así que la polarización en la BC aumenta. En la segunda situación la situación es totalmente opuesta por lo que el filtrado es mínimo y la polarización en la $\mathrm{BC}$ disminuye.

espín opuesta a la mayoría de los portadores generados son atrapados rápidamente por las trampas debido al efecto de filtrado de espín por lo que la eficiencia de polarización de espín en la BC es máxima. Sin embargo, en la segunda situación incidimos el segundo pulso-prueba $\left(P_{p 2}\right)$ en $t_{p 2}=400 \mathrm{ps,}$ donde la polarización electrónica de las trampas debido al primer pulso $\left(P_{0}\right)$ es antiparalela a la polarización de los fotoelectrones generados por $\left(P_{p 2}\right)$, por lo que la eficiencia de polarización es mínima y la cantidad de electrones atrapados por las trampas es máxima. Por consiguiente, las oscilaciones de la polarización electrónica del espín de las trampas producen oscilaciones en el filtrado de espín.

Si bien esta propuesta experimental fue dirigida para los centros para- 
magnéticos en el GaAsN [1], puede ser utilizada para otros semiconductores con impurezas que tengan el mecanismo de la SDR. Por otro lado, aunque es posible incorporar varios isótopos en el modelo, por claridad, nuestros cálculos los desarrollamos con sólo un isótopo promedio.

\subsection{Resultados experimentales y teóricos pa- ra dos isótopos}

Proseguimos nuestra discusión de la respuesta óptica del GaAsN. En esta subsección presentamos los resultados teóricos y experimentales de la dinámica del espín en regimen pulsado considerando en el modelo los dos isótopos estables del Galio $\mathrm{Ga}^{69}$ y $\mathrm{Ga}^{71}$. Para esta variante del modelo consideramos las interacciones de Zeeman de los espines electrónicos de las trampas y de los electrones de la BC. También incluimos la interacción hiperfina entre los espines electrónicos y nucleares de estos dos isótopos. Para finalizar se tomó en cuenta los procesos de relajación del espín en la BC y en las trampas paramagnéticas. El modelo de ecuación maestra lo presentamos en el Apéndice [B].

En la figura (4.5) mostramos los resultados experimentales [2] de la propuesta experimental. Cada punto de la figura representa el máximo de la $S D R_{r}$ del pulso prueba como función del tiempo de retardo $(\Delta t)$ entre el pulso inicial y el pulso prueba. Las mediciones fueron hechas para una potencia del láser $P=4 \mathrm{~mW}$ y a una temperatura $T=7 \mathrm{~K}$. Notamos que la predicción de nuestra propuesta experimental fue acertada. El comportamiento de la $S D R_{r}(\Delta t)$ es sinusoidal evanescente. Es importante enfatizar que los resultados experimentales señalan la participación de al menos dos isótopos estables y por lo tanto exhiben la superposición de dos oscilaciones con frecuencias diferentes. Vemos que para tiempos de retardo $(\Delta t)$ muy largos las oscilaciones pierden visibilidad y observamos una disminución monótona de la $S D R_{r}(\Delta t)$. La línea punteada representa el ajuste de los puntos como la superposición de dos funciones periódicas cuya amplitud decrece exponencialmente.

En las figuras 4.5 (b) y (c) mostramos los espectros de fotolomuniscencia para dos puntos extremos de las oscilaciones, donde las intensidades de la $S D R_{r}(\Delta t)$ son mínima $(\Delta t=150 \mathrm{ps})$ y máxima $(\Delta t=250 \mathrm{ps})$. Notamos que la intensidad del espectro de fotolumniscencia es mayor para $(\Delta t=250$ ps) que para $(\Delta t=150 \mathrm{ps})$. Como lo explicamos en la sección pasada, estas variaciones en la fotolumniscencia se deben a las fluctuaciones presentes en la polarización de espín y de filtrado [1].

UAM-Iztapalapa

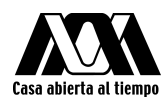



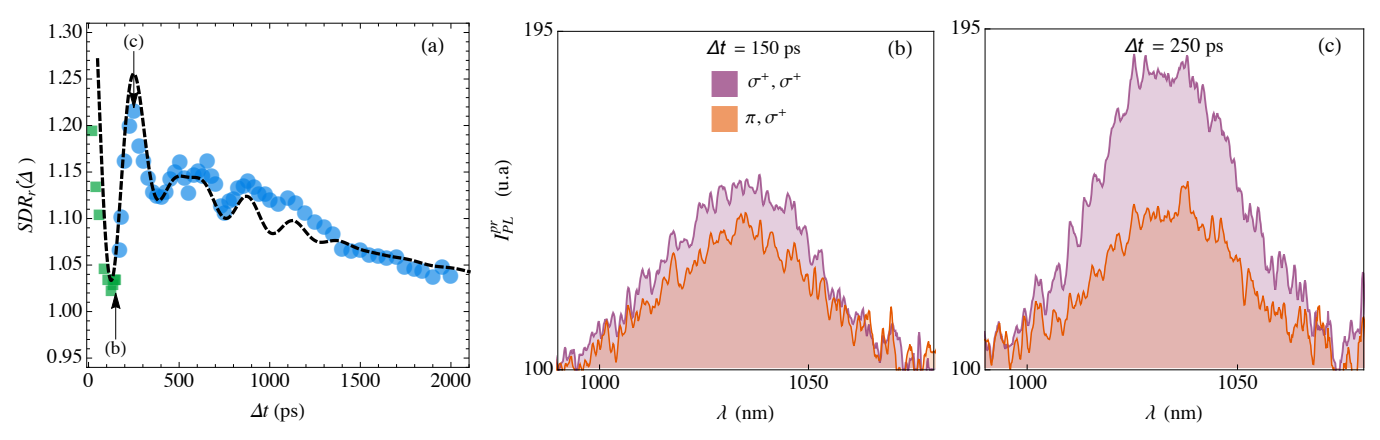

Figura 4.5: En la figura (a) mostramos los resultados experimentales [2] de la propuesta [1]. Cada círculo azul representa el máximo de la $S D R_{r}$ del pulso prueba como función del tiempo de retardo $(\Delta t)$ entre el pulso inicial y el pulso prueba . Las mediciones fueron hechas para una potencia del láser $P=4 m W$ y a una temperatura $T=7 \mathrm{~K}$. El comportamiento de la $S D R_{r}(\Delta t)$ es sinusoidal evanescente. La linea punteada representa el ajuste de los puntos como la combinación lineal de dos funciones cosenos evanescentes ecuación 4.5. Las frecuencias de oscilación son las frecuencias asociadas a las energías de las constantes hiperfinas de los isótopos estables de Galio $\mathrm{Ga}^{69}$ y $\mathrm{Ga}^{71}$. Usamos dos tiempos característicos de decaimiento $\tau_{s c}^{*}$ el tiempo relajación del espín electrónico de las trampas no apareadas y $T_{2}^{*}$ el tiempo de coherencia de las oscilaciones. En las Figuras (b) y (c) mostramos los espectros de fotolumniscencia para tiempos de retado $\Delta t$ de $150 \mathrm{ps}$ y $250 \mathrm{ps}$, respectivamente. Notamos que las intensidades de los espectros cambian, esto se debe a las fluctuaciones del filtrado debido a la interacción hiperfina de los centros paramagnéticos [2].

Con estos resultados, pudimos estimar la concentración relativa de los dos isotopos de Galio $\mathrm{Ga}^{69}$ y $\mathrm{Ga}^{71}$; también estimamos las constantes hiperfinas para cada isótopo. Calculamos estas propiedades sin utilizar campos magnéticos intensos ni microondas. Con el fin de estimar estas propiedades, ajustamos los puntos experimentales de la $S D R_{r}(\Delta t)$ como [2]

$$
\begin{aligned}
& S D R_{r}(\Delta t)-1=\alpha \mathrm{e}^{-\Delta t\left(1 / \tau_{s c}^{*}\right)} \\
& \quad+\beta \mathrm{e}^{-\Delta t / T_{2}}\left[f_{1} \cos \left(\Omega_{1} \Delta t\right)+f_{2} \cos \left(\Omega_{2} \Delta t\right)\right],
\end{aligned}
$$

donde los valores $f_{1}$ y $f_{2}$, son las abundancias relativas de los isótopos estables del Galio $\mathrm{Ga}^{69}$ y Ga $^{71}$. Estos parámetros están normalizados por la condición $f_{1}+f_{2}=1 . \Omega_{1}$ y $\Omega_{2}$ son las frecuencias de las energías de las interacciones hiperfinas para los isótopos $\mathrm{Ga}^{69}$ y $\mathrm{Ga}^{71}$, respectivamente. Cada frecuencia está asociada con la constante hiperfina de cada isótopo, $\mathcal{A}_{i}=\hbar \Omega_{i} / 2$ donde $i=1,2 . \tau_{s c}^{*}$ es el tiempo relajación del espín electrónico de las trampas no apareadas y $T_{2}^{*}$ es tiempo de coherencia de las oscilaciones.

Es importante señalar que los valores de las constantes hiperfinas de los isótopos dependen de la ubicación de los centros paramagnéticos [83] que a su vez depende principalmente de las condiciones del crecimiento del material. 


\begin{tabular}{lcccc}
$\mathrm{Ga}_{i}$ configuración & A & B & C & D \\
\hline $\mathcal{A}_{1}\left({ }^{69} \mathrm{Ga}\right)\left(\times 10^{-4} \mathrm{~cm}^{-1}\right)$ & 745 & 1230 & 620 & 580 \\
$\mathcal{A}_{2}\left({ }^{71} \mathrm{Ga}\right)\left(\times 10^{-4} \mathrm{~cm}^{-1}\right)$ & 968.5 & 1562 & 787.4 & 736.6
\end{tabular}

Cuadro 4.1: Tabla de las constantes hiperfinas para los dos isótopos estables de Galio $\left(\mathrm{Ga}^{69} \mathrm{y} \mathrm{Ga}^{71}\right)$ en los cuatro configuraciones intersticiales del (In)GaAsN. 83.

Sabemos que hay cuatro posibles configuraciones para los isótopos de $\mathrm{Ga}^{69}$ y $\mathrm{Ga}^{71}$ (Cuadro 4.1).

En la Figura 4.6 graficamos la $S D R_{r}(\Delta t)$ para un intervalo del tiempo de retraso de $[0,1000]$ picosegundos. Con el fin de obtener las constantes hiperfinas de los isótopos, superponemos cuatro curvas que representan la combinación lineal de $\cos \left(\Omega_{1}\right)$ y $\cos \left(\Omega_{2}\right)$, donde las $\Omega_{\mathrm{s}}$ son las frecuencias de los isótopos $\mathrm{Ga}^{69}$ y $\mathrm{Ga}^{71}$, respectivamente.

Los puntos azules en la Figura 4.6 representan los datos experimentales. Estos puntos muestran una interferencia destructiva entre 500 ps y 600 ps, lo cual excluye a las configuraciones de $\mathrm{Ga}_{i}-\mathrm{A}$ y $\mathrm{Ga}_{i}$-B, ya que las curvas de estas configuraciones no tienen este comportamiento a causa de sus altas frecuencias de oscilación. En particular, notamos que las constantes hiperfinas de las configuraciones de $\mathrm{Ga}_{i}{ }^{-} \mathrm{C}$ y $\mathrm{Ga}_{i}{ }^{-} \mathrm{D}$ son muy similares 4.1. Sin embargo, notamos que los máximos y mínimos de la curva para $\mathrm{Ga}_{i}$-D están en las mismas posiciones en $(\Delta t)$ que los valores maximales de los puntos experimentales. De modo que las frecuencias utilizadas para los isótopos en la muestra son $\Omega_{1}=2.18 \mathrm{GHz}$ y $\Omega_{1}=2.75 \mathrm{GHz}$ por consiguiente las constantes hiperfinas son $\mathcal{A}_{1}=577.8 \times 10^{-4} \mathrm{~cm}^{-1}$ y $\mathcal{A}_{2}=730.6 \times 10^{-4} \mathrm{~cm}^{-1}$. Con estos valores y la ecuación (4.5), por un lado, encontramos que los valores para las abundancias relativas son $f_{1}=0.59$ y $f_{2}=0.41$. Por otro lado, hallamos que $\tau_{s c}^{*}=1350$ ps y $T_{2}^{*}=350$ ps.

Para finalizar, en la Figura 4.7 mostramos los resultados de la $S D R_{r}(\Delta t)$ en ausencia y en presencia de un campo magnético de $B=65 \mathrm{mT}$ en configuración de Faraday, por lo que la dirección del campo magnético es paralela a la dirección de polarización. En la figura 4.7 (a), los círculos morados y azules representan los puntos experimentales de la $S D R_{r}(\Delta t)$ para los casos en que $B=0 \mathrm{mT}$ y $B=65 \mathrm{mT}$, respectivamente. En la figura 4.7 (b), los triángulos 


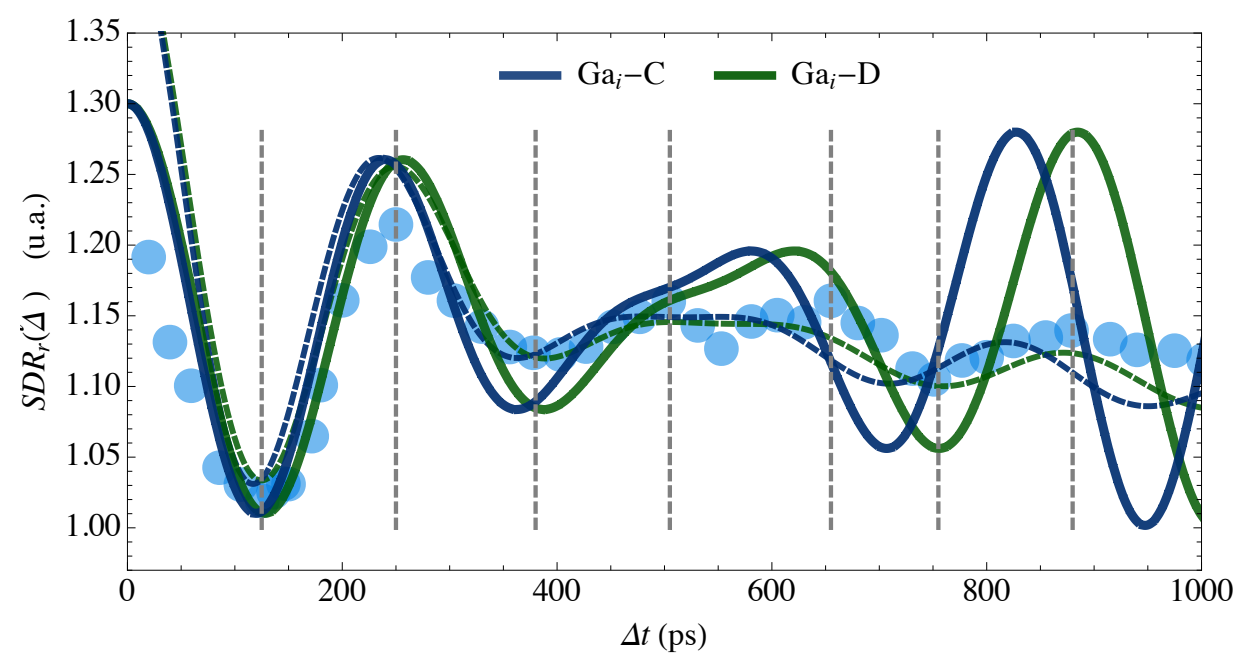

Figura 4.6: Los puntos azules representan los datos experimentales para un intervalo de $\Delta t[0,1000]$ ps. Las líneas azul y roja son funciones senoidales que representan las oscilaciones de la superposición de los dos isótopos $\mathrm{Ga}^{69}$ y Ga ${ }^{71}$ sin amortiguamiento en las configuraciones $\mathrm{Ga}_{i}-C \mathrm{y} \mathrm{Ga}_{i}-D$, respectivamente. Las líneas punteadas representan las funciones senoidales amortiguadas descritas por $T_{2}^{*}$. Las líneas verticales punteadas señalan la posición de los máximos y mínimos de los puntos experimentales.

morados y azules representan los puntos teóricos de la $S D R_{r}(\Delta t)$ usando el modelo [2] para los casos en que $B=0 \mathrm{mT}$ y $B=65 \mathrm{mT}$, respectivamente (Apéndice B) . Las líneas punteadas en ambas figuras representan el ajuste de las $S D R(\Delta t)$ usando la combinación lineal de dos funciones periódicas evanescentes que vimos anteriormente en la ecuación (4.5).

Primero notamos que las curvas formadas por los puntos obtenidos con el modelo Figura 4.7 (b), tienen el mismo comportamiento y tienen las mismas intensidades que las curvas formadas por los puntos experimentales Figura 4.7 (b), para los casos en que el campo magnético es $B=0 \mathrm{mT}$ y $B=65 \mathrm{mT}$. Segundo, nos percatamos que las curvas con y sin campo magnético tienen tres principales diferencias. La primera, la intensidad de la $S D R_{r}(\Delta t)$ para $B \neq 0$ es mayor que para el caso en que $B=0$. La segunda, las oscilaciones debidas al efecto de flip-flop de espín electrón-núcleo presentes en $B=0$ desaparecen para $B \neq 0$. Tercero, la relajación de espín en $B \neq 0$ es mucho más lenta que en $B=0$.

En general, estas observaciones sugieren que la presencia de un campo magnético en configuración de Faraday estabiliza la polarización electrónica en la BC. Esto se debe a que la interacción Zeeman de los espines electrónicos en la $\mathrm{BC}$ y en las trampas domina sobre la interacción hiperfina que hay en las trampas paramagnéticas lo que inhibe la transferencia de espín entre los electrones y núcleos ligados. Así, el espín electrónico se desacopla del espín 

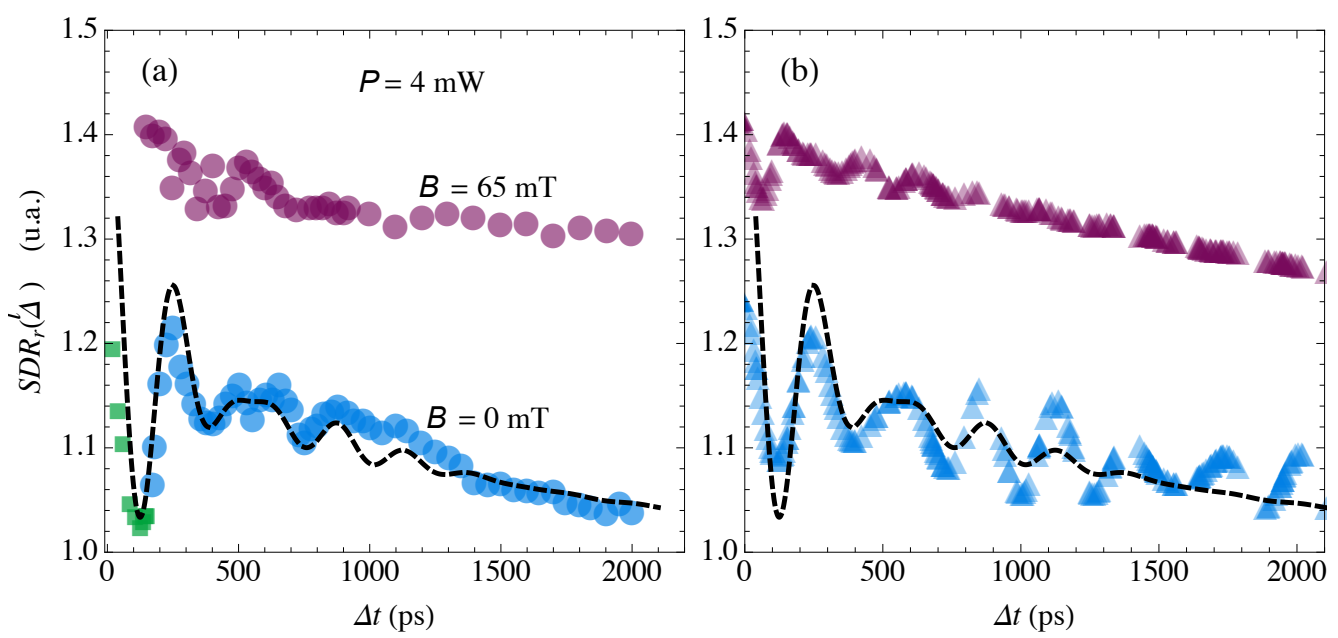

Figura 4.7: Razón de recombinación dependiente del espín en función del tiempo de retardo $(\Delta t)$ entre el pulso y el pulso-prueba. En el panel (a), los círculos representan los resultados experimentales y en el panel (b), los triángulos representan los resultados de nuestro modelo (Apéndice B). Las figuras de color azul son la $S D R_{r}(\Delta t)$ para $B=0$ y las figuras de color morado son la $S D R_{r}(\Delta t)$ para $B=65 \mathrm{mT}$. Las líneas punteadas en las dos figuras representan el ajuste de los resultado experimentales ( eucación 4.5) para el caso en que $B=0$. Los puntos experimentales y los calculados por el modelo tienen las mismas intensidades y el mismo comportamiento para los casos con y sin campo magnético.

nuclear. Dado que los electrones ligados en las trampas poseen tiempos de relajación de espín muy largos en presencia de un campo magnético externo longitudinal ( $\tau_{s c}>200 \mathrm{~ns}$ ), está claro que, a medida que el campo magnético aumenta la IHF se desacopla por la interacción de Zeeman, el espín electrónico de las trampas se relaja lentamente debido a $\tau_{s c}$. Por el contrario, cuando el campo magnético es insignificante y, en consecuencia, los electrones y núcleos en las trampas están fuertemente acoplados por el IHF, los espines de los electrones en las trampas decaen más rápido a medida que su momento angular se transfiere a los espines nucleares que se relajan rápidamente.

\subsection{Régimen pulsado en presencia de un cam- po magnético oblicuo a la dirección de polarización}

Debido a las acertadas predicciones del modelo [2], ahora, nuestra intención es mostrar que es posible detectar y trazar las polarizaciones electronicas 
y nucleares en las tres direcciones espaciales.

En esta sección, investigamos de manera teórica una variación de la propuesta experimental antes mencionada con el enfoque de la ecuación maestra [1]. La hipotesis se centra en la posibilidad de caracterizar la dinámica del espín tridimensional usando el grado de polarización circular de la fotoluminiscencia en lugar de la $S D R_{r}$.

Cabe mencionar que la $S D R_{r}$ utilizada en las ref. [1, 2] no tiene resolución espacial así que en este caso proponemos detectar el grado de polarización circular. Esto se puede lograr de dos maneras. En la configuración de retrodispersión, donde la excitación y la detección se realizan a lo largo del mismo eje (eje $z$ ), el grado de polarización circular se determina a lo largo de la línea de excitación. Para medir el grado de polarización circular a lo largo de los otros dos ejes $(x$ e $y$ ) se deben obtener los parámetros de Stokes midiendo el grado de polarización lineal a lo largo de dos marcos ortogonales diferentes, $(x, y)$ y $\left.\left(x^{\prime}, y^{\prime}\right)\right)$ girado a $45^{\circ}$. Esto determina los grados de polarización circular a lo largo de las direcciones $x \& y$. Otro método consiste en colocar directamente la detección a lo largo de cada dirección.

Por otro lado, para inducir polarización de espín en las tres direcciones espaciales, necesitamos aplicar un campo magnético oblicuo a la dirección de polarización. A pesar de que el campo magnético induce su propia dinámica del espín, algunas de las características observadas pertenecen exclusivamente a las relajaciones del espín y a la interacción hiperfina.

Centramos esta discusión en el cálculo del promedio de las tres componentes de los grados de polarización de espín de los electrones en la BC.

$$
\operatorname{Pei}(\Delta t)=200 \frac{\left\langle\bar{S}_{i}(t)\right\rangle}{\langle\bar{n}(t)\rangle},
$$

donde $i=x, y, z$ y los $\langle\ldots\rangle$ brackets es el promedio temporal. Consecuentemente

$$
\begin{aligned}
\left\langle\bar{S}_{i}(t)\right\rangle(t) & =\frac{1}{T} \int_{\Delta t}^{T} \bar{S}_{i}(t) d t \\
\langle\bar{n}(t)\rangle(t) & =\frac{1}{T} \int_{\Delta t}^{T} \bar{n}(t) d t
\end{aligned}
$$

son los promedios temporales de las componentes de espín y el número de electrones en la banda de conducción. En la ecuación (4.6) el factor 200 es necesario para parametrizar el grado de polarización de espín entre $-100 \%$ y $100 \%$ para los electrones completamente polarizados en los estados $S_{z}=$ $-\hbar / 2$ y $S_{z}=\hbar / 2$.

La prueba se hizo para $P=4 \mathrm{~mW}$ en ausencia de campo magnético. En la Figura 4.8 (a) mostramos el grado de polarización de espín Pez creado 

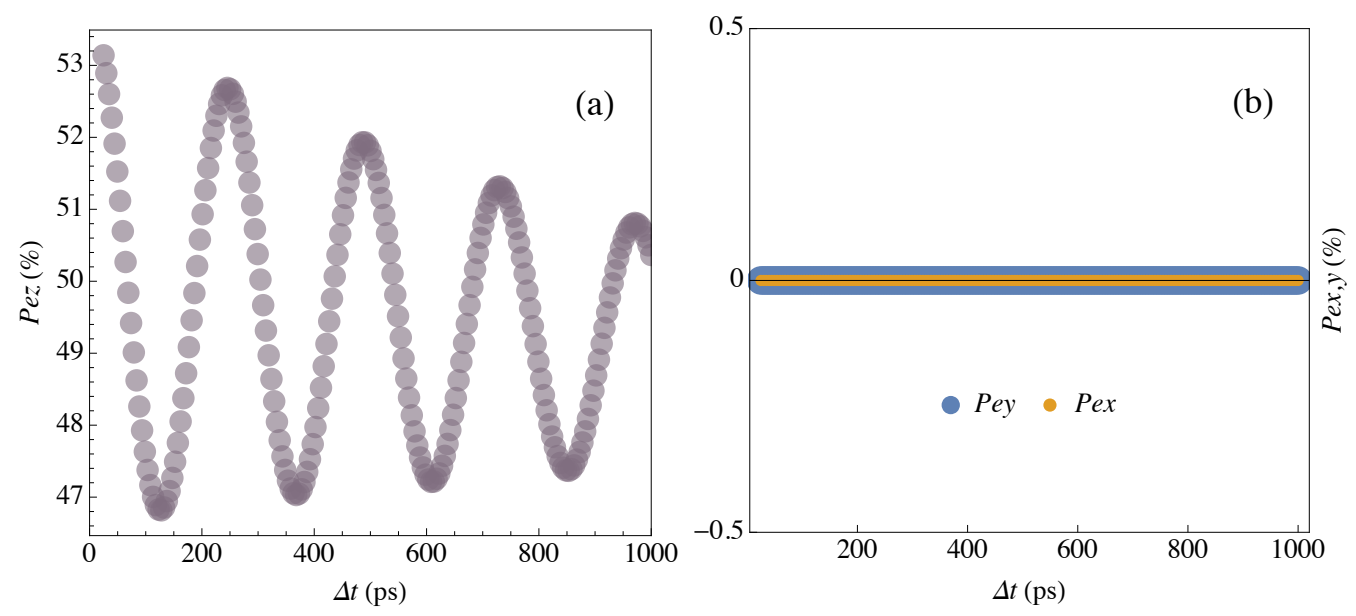

Figura 4.8: La polarización de espín de banda de conducción creado por el pulso prueba como función del tiempo de retardo $\Delta t$ entre el pulso inicial y el pulso prueba para $P=$ $4 \mathrm{~mW}$. (a) Grado de polarización Pez como una función de $\Delta t$. (b) Las componentes transversales del grado de polarización de espín Pex y Pey como función de $\Delta t$.

por el pulso prueba como función del tiempo de retraso $\Delta t$. Notamos que las fluctuaciones en el efecto de filtrado del espín estan presentes en la polarización. Las componentes de la polarización de los electrones en la BC Pex, Pey y $P e z$ pueden ser directamente ligadas a $S_{x}, S_{y}$ y $S_{z}$. En la Figura 4.8 (b) mostramos Pex y Pey como función $\Delta t$. Como esperamos, despues de haber generando polarización de espín en la dirección $z$ y en ausencia de un campo magnético externo tenemos que ambas cantidades son cero durante todo el tiempo.

A continuación, mostramos como influyen las intensidades de la potencia del laser y un campo magnético externo en la polarización electrónica del espín en la BC.

\subsubsection{Optimización de la potencia}

Primero estudiamos la relación que hay entre las intensidades de las potencias del primer pulso y del pulso-prueba con la polarización de los electrones de la BC. Con el fin de realizar este análisis, introdujimos un parámetro $\alpha$ a la expresión de la generación de electrón-hueco

$$
G(t)_{ \pm}=\frac{G_{0} P t_{p p}}{2 \sqrt{2 \pi} s_{t}}\left[\left(1 \pm P_{e 1}\right) \mathrm{e}^{-\frac{1}{2}\left(\frac{t-t_{10}}{s_{t}}\right)^{2}}+\alpha\left(1 \pm P_{e 2}\right) \mathrm{e}^{\left.-\frac{1}{2}\left(\frac{t-t_{20} \delta t}{s_{t}}\right)^{2}\right]}\right.
$$

donde el parametro $\alpha=P_{p} / P_{1}$ es la razón entre las potencias del pulso prueba $\left(P_{p}\right)$ y el primer pulso $\left(P_{1}\right)$. 
Consideramos además tres valores de $\alpha=1,100$ y 0.001. Por un lado, los casos en que $\alpha \neq 1$ las potencias de los pulsos son de $P=4 \mathrm{~mW}$. Por otro lado, cuando $\alpha=1$ consideramos dos casos cuando $P=4 \mathrm{~mW}$ y $P=40 \mathrm{~mW}$.

En la Figura 4.9, mostramos el grado de polarización de espín Pez como función del tiempo de retardo $(\Delta t)$ entre el primer pulso y el pulso-prueba para diferentes combinaciones de potencia. Los dos paneles superiores [(a) y (b)] muestran los casos en que los pulsos tienen las mismas intensidades $(\alpha=1)$ pero potencias de $P=4 \mathrm{~mW}$ y $P=40 \mathrm{~mW}$, respectivamente. Los paneles (c) y (d) muestran las situaciones extremas donde $\alpha=100 \mathrm{y}$ $\alpha=0.001$ con una potencia de $P=4 \mathrm{~mW}$. En el panel (e) mostramos las curvas de polarización electrónica y nuclear de las trampas no apareadas.

Notamos que las curvas de polarización del espín Pez extraído de las pruebas de fotoluminiscencia reproducen las características reales del espín $S_{c z}$ en función del tiempo.

Con el fin de inferir cual es la configuración más óptima de las intensidades del pulso y del pulso-prueba, en la Figura 4.10 graficamos la diferencia entre los máximos y mínimos $(\delta A)$ de cada gráfica del grado de polarización de espín $P e z$. Los puntos rojos, negros, azules y naranjas son los casos en que $\alpha=1$ y $P=4 \mathrm{~mW}, \alpha=1$ y $P=40 \mathrm{~mW}, \alpha=100$ y $P=4 \mathrm{~mW}$, y $\alpha=0.001$ y $P=4 \mathrm{~mW}$.

De la Figura 4.10 inferimos que la intensidad más grande del grado de polarización circular del pulso-prueba $P e z$ es cuando los dos pulsos tienen la misma intensidad $\alpha=1$. Este comportamiento es repetido consistentemente por otros valores de potencia (no se muestran aquí).

Ahora estudiamos las intensidades de los pulsos en presencia de un campo magnético externo en configuración de Voigt, es decir que el campo magético esta en la dirección perpendicular al eje de polarización. Para este caso consideramos que la polarización fue hecha en la dirección $z$ y que el campo esta en la dirección $x$.

En la Figura 4.11 mostramos las polarizaciones $\operatorname{Pez}(\Delta t), \operatorname{Pey}(\Delta t)$ y $\operatorname{Pex}(\Delta t)$, en presencia de un campo magnético externo en configuración de Voigt como función del tiempo de retardo $(\Delta t)$ entre el primer pulso y el pulso-prueba para las mismas configuraciones de potencia de los pulsos de la Figura 4.9. Los paneles [(a) y (b)] muestran los casos en que los pulsos tienen las mismas intensidades $(\alpha=1)$ pero potencias de $P=4 \mathrm{~mW}$ y $P=40 \mathrm{~mW}$, respectivamente. Los paneles (c) y (d) muestran las situaciones extremas donde $\alpha=100$ y $\alpha=0.001$ con una potencia de $P=4 \mathrm{~mW}$. En los paneles (e) mostramos las curvas de polarización electrónica y nuclear de las trampas no apareadas.

Notamos que las curvas de polarización del espín Pez, Pey y Pex extraí- 

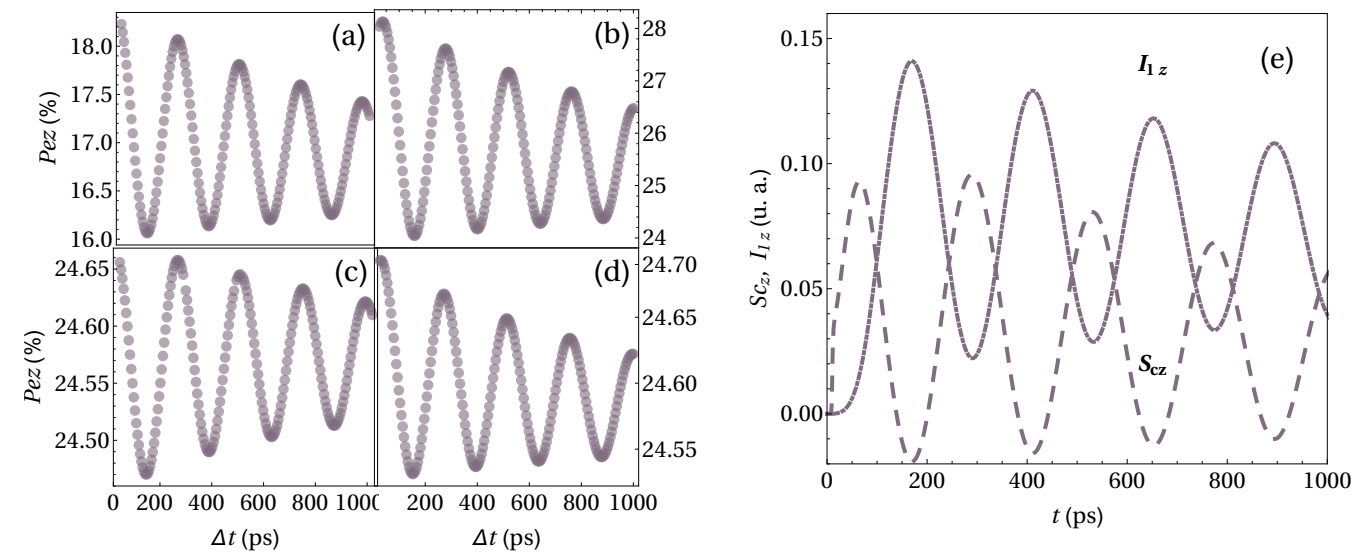

Figura 4.9: Grado de polarización de espín $P_{e z}$ creado por el pulso prueba como función del tiempo de retraso $\delta t$ para diferentes combinaciones de la potencia del pulso y del pulso prueba para $B=0$. (a) $P=4 \mathrm{~mW}$ y $\alpha=1$, (b) $P=40 \mathrm{~mW}$ y $\alpha=1$, (c) $P=0.4 \mathrm{~mW}$ y $\alpha=100$, (d) $P=40 \mathrm{~mW}$ y $\alpha=0.001$. (e) La dependencia temporal de $\bar{S}_{c z}$ y $\bar{I}_{1 z}$.

das de las pruebas de fotoluminiscencia reproducen las características reales del espín $S_{c z}, S_{c y}$ y $S_{c x}$ en función del tiempo.

En la Figura 4.12 mostramos la diferencia entre los máximos y mínimos de las curvas de polarización Pez, Pey y Pex en configuración de Voigt para las diferentes configuraciones de la potencia de los pulsos de la Figura 4.11. Estos resultados confirman que incluso en presencia de un campo magnético perpendicular a la dirección de polarización, la mejor configuración es que los dos pulsos tengan la misma intensidad de potencia $(\alpha=1)$.

Comparando las Figuras 4.9 y 4.11, notamos que las amplitudes de Pez tiene una rápida razón de amortiguamiento en presencia de un campo magnético perpendicular como un resultado de la despolarización inducida por el efecto Hanle. Notamos que la polarización no es nula a bajos campos magnéticos. Por simplicidad, para el estudio de la polarización del espín en materiales es preferible usar pequeños campos magnéticos que intensos. En contraste con el caso de $B=0$ donde $P e x=P e y=0$ Figura 4.8, cuando $B_{x}=10 \mathrm{mT}$ Figura 4.11 $P_{e x}$ y $P_{e y}$ exhiben oscilaciones cuyas frecuencias son consistentes con las oscilaciones hiperfinas.

Los paneles (e) de la Figura 4.11 muestran la dependencia temporal de las componentes de espín electrónico de las trampas $\bar{S}_{c x}(t), \bar{S}_{c y}(t), \bar{S}_{c y}(t)$ y de espín nuclear $\bar{I}_{1 x}(t), \bar{I}_{1 y}(t)$ y $\bar{I}_{1 z}(t)$. Notamos que la tendencia de estas gráficas es la misma que la de las polarizaciones $\operatorname{Pex}(\Delta t), \operatorname{Pey}(\Delta t)$ y $\operatorname{Pez}(\Delta t)$ mostradas en los paneles (a), (b), (c) and (d) de la Figura 4.11. En principio estas polarizaciones pueden ser medidas experimentalmente. Entonces, 


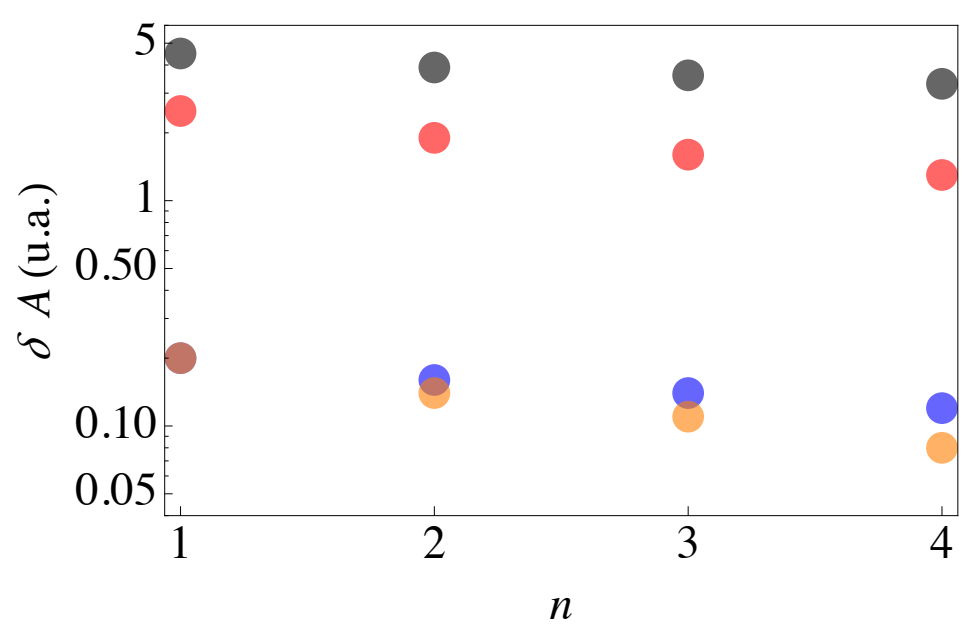

Figura 4.10: Diferencia entre los máximos y mńimos de las curvas de grado de polarización de espín Pez de la Figra 4.9. Los puntos rojos, negros, azules y naranjas son los casos en que $\alpha=1$ y $P=40 \mathrm{~mW}, \alpha=1$ y $P=4 \mathrm{~mW}, \alpha=100$ y $P=4 \mathrm{~mW}$, y $\alpha=0.001$ y $P=4 \mathrm{~mW}$.

la evolución temporal de las componentes de espín de los electrones ligados $\bar{S}_{c x}(t), \bar{S}_{c y}(t)$ y $\bar{S}_{c z}(t)$ pueden ser monitoreadas por los grados de polarización de espín $P e x(\Delta t), \operatorname{Pey}(\Delta t)$ y $P e z(\Delta t)$.

Es importante señalar que debido a los resultados de las componentes espaciales de los grados de polarización de espín y los resultados teóricos para $\bar{I}_{1, x, y, z}(t)$, las caracteristicas de la variación temporal del espín nuclear pueden ser inferidas con las curvas $P_{e x}(\Delta t), P_{e y}(\Delta t)$ y $P_{e z}(\Delta t)$.

En la Figura 4.13 (a) mostramos la polarización en el eje x Pex en presencia de un campo magnético en configuración Voigt para los casos en que tomamos en cuenta la interacción hiperfina y cuando no esta presente dicha interacción. Notamos que para el caso en que $\mathrm{IHF}=0$ la polarización $P e x$ desaparece contrario cuando $\mathrm{IHF} \neq 0 \mathrm{Pex}$. Cuando tomamos en cuenta la interacción hiperfina, Pex tiene un comportamiento oscilante con una intensidad muy pequeña. A pesar de que la polarización en la dirección x es mínima es una firma de la interacción hiperfina. Por lo tanto, vale la pena intentar mejorar los valores de $P_{e x}(\Delta t)$. Para hacerlo, podemos cambiar la dirección del campo magnético para maximizar $P_{e x}(\Delta t)$.

\subsubsection{Optimización del campo magnético}

Con el fin de obtener el valor de la dirección óptima del campo magnético para obtener la $\operatorname{Pex}(\Delta t)$ más intensa, calculamos la $\operatorname{Pex}(\Delta t)$ para diferentes valores de $\theta$, donde $\theta$ es el ángulo entre la dirección de polarización inicial 

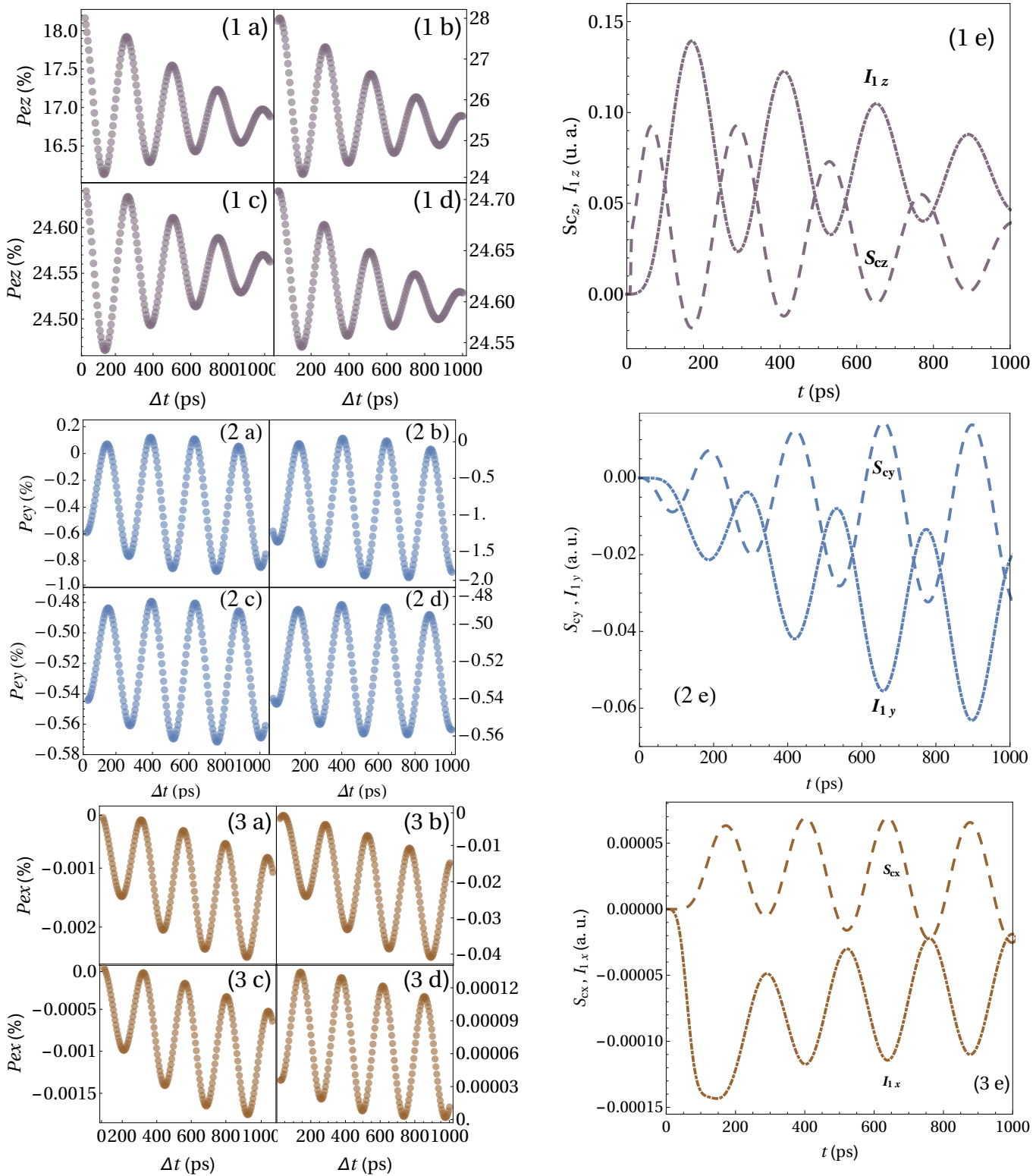

Figura 4.11: Grado de polarización de espín para las tres componentes espaciales $P_{e z}$, $P_{e y}$ y $P_{e x}$ como una función del tiempo de retraso $\Delta t$ para diferentes combinaciones de la potencia del pulso y el pulso prueba para $B_{x}=10 \mathrm{mT}$. (a) $P=4 \mathrm{~mW}$ y $\alpha=1$, (b) $P=40 \mathrm{~mW}$ y $\alpha=1$, (c) $P=0.4 \mathrm{~mW}$ y $\alpha=100$, (d) $P=40 \mathrm{~mW}$ y $\alpha=0.001$. (e) La dependencia de temporal de las tres componentes espaciales de espín electrónico $\bar{S}_{c z}, \bar{S}_{c y}$, $\bar{S}_{c x}$ y nuclear $\bar{I}_{1 z}, \bar{I}_{1 y}, \bar{I}_{1 x}$.

y el eje $x$. En la Figura 4.13 mostramos $\operatorname{Pex}(\Delta t)$ para varias orientaciones del campo magnético. En el recuadro dentro de la Figura 4.14 graficamos la evolución temporal de $\operatorname{Pex}(\Delta t)$ para un periodo más largo de tiempo 


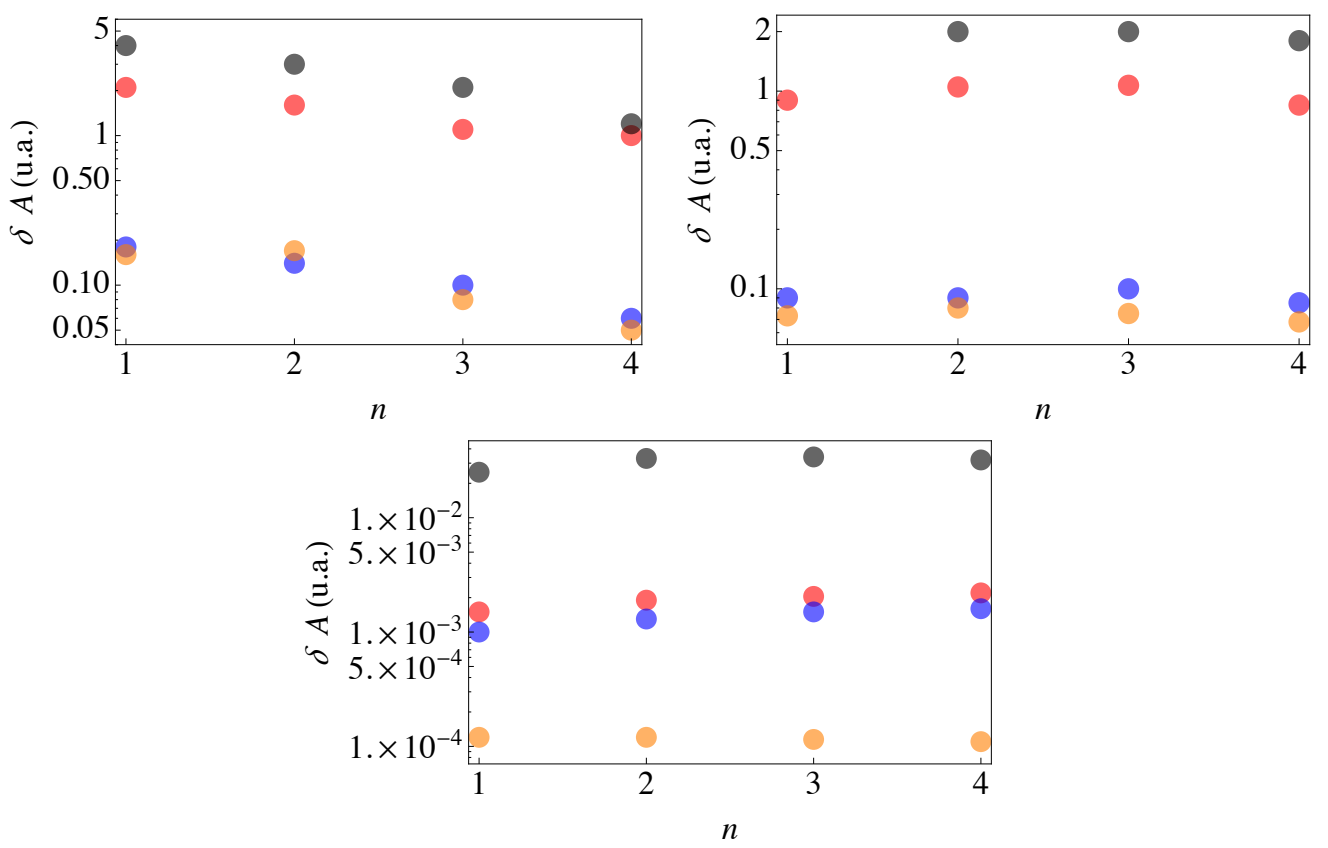

Figura 4.12: Diferencia entre los máximos y mńimos de las curvas de grado de polarización de espín Pez, Pey y Pex de la Figra 4.9 Los puntos rojos, negros, azules y naranjas son los casos en que $\alpha=1$ y $P=40 \mathrm{~mW}, \alpha=1$ y $P=4 \mathrm{~mW}, \alpha=100$ y $P=4 \mathrm{~mW}, \mathrm{y}$ $\alpha=0.001$ y $P=4 \mathrm{~mW}$.

revelando que las oscilaciones rápidas tienen un periodo de $T \approx 200$ ps debido a la interacción hiperfina. Estas oscilaciones se modulan por la precesión lenta del espín debido a la interacción de Zeeman con un periodo de $T \approx 2$ ns. Notamos que la orientación óptima del campo magnético se observa para $\theta=45^{\circ}$ donde $\operatorname{Pex}(\Delta t)$ presenta la amplitud máxima.

Para finalizar, en la figura $4.15 \operatorname{Pex}(\Delta t)$ mostramos las curvas con y sin [IHF un angulo de inclinación de $\theta=45^{\circ}$ con referente a la dirección de polarización. Este panel muestra que un campo magnético orientado a $45^{\circ}$ con respecto a la luz incidente no solo revela las características principales de la IHF en los centros $\mathrm{Ga}^{2+}$, sino que también mejora la intensidad de $\operatorname{Pex}(\Delta t)$ a fin de que sea más fácil su detección. 


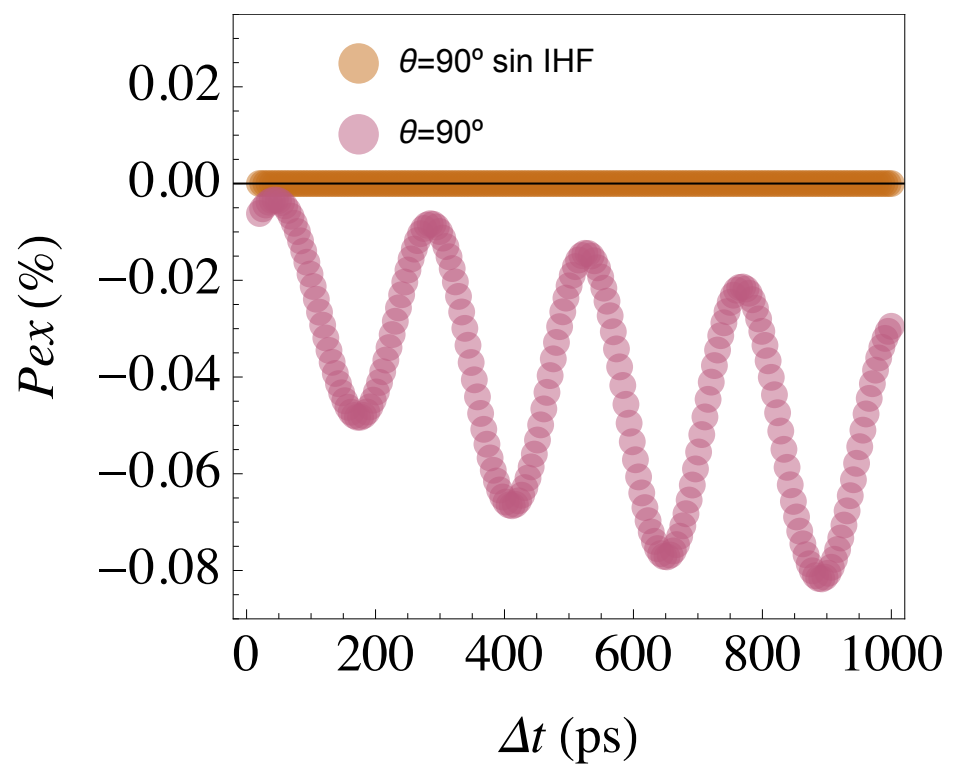

Figura 4.13: Comparación de la polarización $P_{e x}$ en configuración de Voigt cuando IHF es cero (circulos rojos) y cuando tomamos en cuenta la interacción hiperfina. 


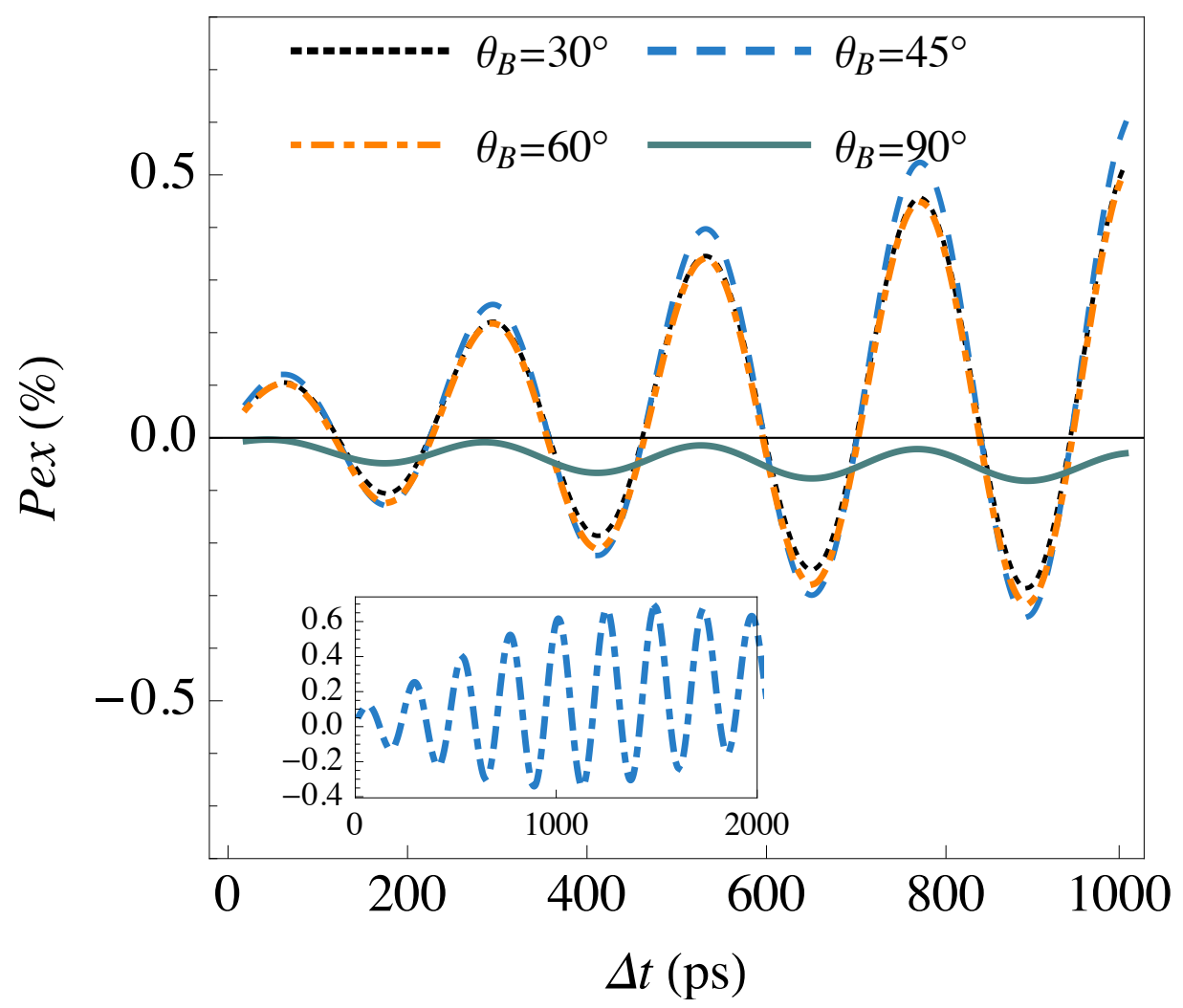

Figura 4.14: Grado de polarización de espín Pex como una función del tiempo de retraso $\Delta t$ entre el pulso y el pulso prueba para varios orientaciones del campo magnético. Los ángulos son medidos con respecto a la dirección de luz incidente. Las curvas para las orientaciones del campo magnético $\theta_{B}=30^{\circ}, 45^{\circ}, 60^{\circ}, 90^{\circ}$ son mostradas. Todas las curvas son calculadas para $B=10 \mathrm{mT}, P=40 \mathrm{~mW}$ y $\alpha=1$. El inset muestra $P e x$ para tiempos de retraso más largos. 


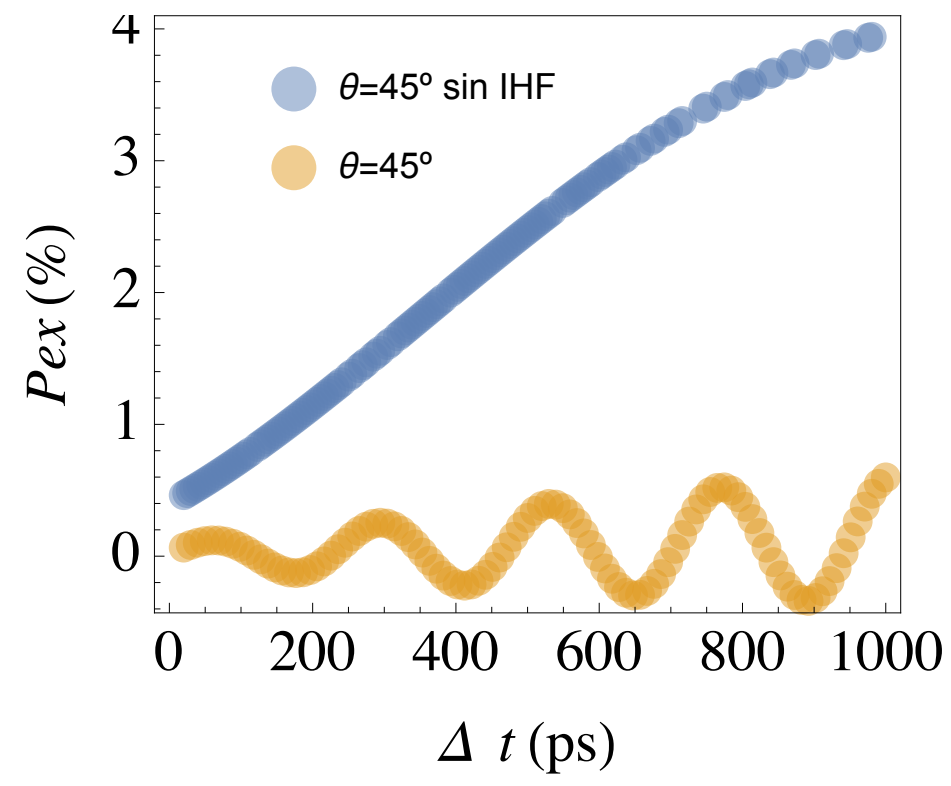

Figura 4.15: Polarización $P_{e x}$ en presencia de un campo magnético externo en con un angulo de inclinación de $\theta=45^{\circ}$ con referente a la dirección de polarización. El comportamiento oscilante de la curva naranja se debe al flip-flop de los centros paramagnéticos debido al acomplamiento hiperfino del espín nuclear y el espín electrónico de las trampas paramagnéticas. 


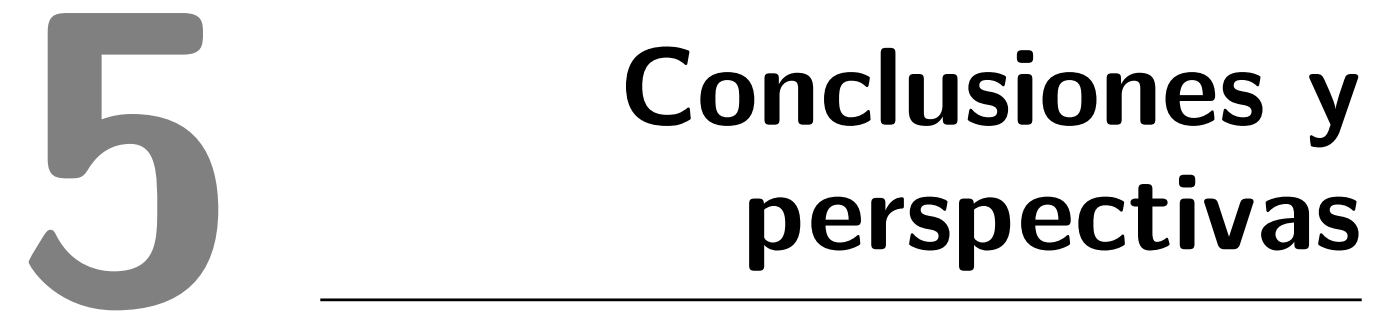

$\mathrm{E}$ n este trabajo presentamos un estudio teórico de la dinámica del espín de los centros paramagéticos $\mathrm{Ga}^{2+}$ en el GaAsN en régimen pulsado. Desarrollamos un modelo con el enfoque de la ecuación maestra capaz de describir la dinámica del espín electrónico y nuclear de los $\mathrm{Ga}^{2+}$ en el GaAsN en régimen pulsado en ausencia de un campo magnético externo y en la presencia de un campo magnético en las configuraciones de Voigt y de Faraday, así como en presencia de un campo magnético oblicuo a la dirección de polarización.

El modelo que construimos tiene tres principales elementos; la matriz de densidad, el hamiltoniano y el disipador. La matriz de densidad está formada por los estados cuánticos de los huecos en la banda de valencia, los electones en la banda de conducción, las trampas paramagnéticas no apareadas y las trampas apareadas. La base de operadores que describen al sistema tiene un álgebra de Lie fínita de dimensión 85. El hamiltoanio del sistema contiene las interacciones de Zeeman de los electrones de la banda de conducción, de Zeeman de los electrones ligados a la trampas paramagnéticas y la interacción hiperfina ente los espines electrónico y nuclear de los centros paramagnéticos. El disipador contiene todas las interacciones del sistema con sus alrededores como son el mecanismo de la recombinación dependiente del espín extraído del modelo de dos cargas; los mecanismos de relajación de espín electrónica en la banda de conducción y en las trampas paramagnéticas; los mecanismos de relajación nuclear de las trampas apareadas y no apareadas y el mecanismo de generación de electrón-hueco e inyección del espín en la banda de conducción. Para introducir los mecanismos de relajación del espín electrónico y del espín nuclear usamos la teoría de Wangness-Bloch-Redfield.

Con el fin de extraer información del modelo, construimos un sistema de de ecuaciones que proviene de la ecuación maestra. Introducimos el Hamiltoniano, la matriz de densidad y el disipador en la ecuación maestra. Con la ayuda de la traza obtenemos un sistema de 85 ecuaciones no lienales de primer orden. Con este sistema de ecuaciones, estudiamos la dinámica del espín 
de los electrones y de los núcleos. Construimos y resolvimos númericamente dicho sistema con un algoritmo hecho en Mathematica [82]. El sistema de 85 ecuaciones diferenciales no lineales de primer orden solo tiene 8 parámetros libres que son: $\gamma_{r}$ la razón de recombinación bimolecular entre la $\mathrm{BC}$ y la $\mathrm{BV} ; \gamma_{e} \mathrm{y} \gamma_{h}$ son las razones de recombinación para los electrones en la BC y los huecos en la BV, $N_{c}$ el número total de centros $\mathrm{Ga}^{2+} ; \tau_{s}$ y $\tau_{s c}$ son los tiempos de relajación del espín para los electrones de la BC y de las trampas no apareadas; $\tau_{n, 1}$ y $\tau_{n, 2}$ son los tiempos de relajación de espín nuclear para las trampas no apareadas y para las trampas apareadas, respectivamente.

La principal contribución de este trabajo radica en la posibilidad de detectar y trazar el flip-flop del espín electrón-núcleo debido a la interacción hiperfina en los centros paramagnéticos en el GaAsN a través de la medición de la fotoluminiscencia de banda a banda en ausencia de un campo magnético externo.

Con la ayuda de la razón de recombinación dependiente del espín mostramos que es posible detectar la polarización electrónica del GaAsN en presencia de un pulso electromagnético. Asimismo, mostramos que con la presencia de un segundo pulso consecutivo es posible detectar la información de la dinámica desatada por el primer pulso. En este caso las pruebas se realizaron sin campo magnético y demostramos que las intensidades de los pulsos deben ser iguales para incrementar la señal detectada.

Debido al comportamiento de la razón de recombinación dependiente del espín en régimen pulsado, propusimos un experimento de pulso-prueba, el cual consiste en hacer una serie de pruebas con dos pulsos consecutivos con el fin de medir la razón de recombinación dependiente del espín. Para cada evento o prueba aumentamos el espaciamiento temporal entre los dos pulsos. Los resultados teóricos mostraron que es posible trazar el flip-flop de espín electrón-núcleo de los centros paramagnéticos [1].

Es importante notar que las predicciones del modelo y de la propuesta experimental fueron corroborados [2]. Con los datos del flip-flop de espín de los centros pudimos determinar las constantes hiperfinas y las abundancias relativas de los núcleos involucrados sin la necesidad de las técnicas de resonancia de espín electrónica que son mucho más complicadas. La información de los parámetros de relajación del espín electrónico y nuclear también fueron determinados a partir de los datos de las oscilaciones evanescentes debido al flip-flop de espín del electrón-núcleo de los centros paramagnéticos. Los parámetros son directamente atribuidos a los mecanismos de relajación de espín nuclear y electrónico bajo un moderado campo magnético de $65 \mathrm{mT}$ en la configuración de Faraday; el comportamiento oscilatorio típico de la interacción hiperfina se apaga y el efecto de filtrado de espín es más eficiente. Los

UAM-Iztapalapa

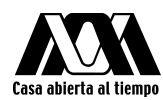


espines de los electrones ligados en los centros $\mathrm{Ga}^{2+}$ son fuertemente estabilizados por el campo magnético externo a través de la interacción Zeeman el cual los desacopla del espín nuclear y evita la transferencia del momento angular entre los dos espines.

Por otro lado, basado en datos experimentales previos [2] y resultados teóricos [1], hemos mostrado que es posible monitorear la evolución temporal de las componentes de espín electrónico y nuclear de los centros $\mathrm{Ga}^{2+}$ en el GaAsN por medio de un esquema de pulso prueba bajo la acción de un campo magnético inclinado $(10 \mathrm{mT})$ [3].

Las condiciones óptimas para tales experimentos fueron determinadas. Primero, las intensidades del primer pulso y el pulso-prueba deben ser iguales con el fin de maximizar la amplitud del grado de polarización de espín. Segundo, la intensidad del campo magnético debe ser de unos pocos $\mathrm{mT}$ y debe de tener un ángulo de inclinación de $45^{\circ}$ con respecto a la dirección de la polarización de la luz incidente.

Adicionalmente, mostramos que los resultados teóricos tienen el mismo comportamiento de los posibles datos experimentales del grado circular de polarización. Así que es posible obtener las principales características de la dinámica del espín nuclear.

En un futuro trabajo, a través de una simple modificación $\left(B_{x} \rightarrow B_{x}+\right.$ $B_{0} \sin \omega t$ ), el modelo puede ser usado para estudiar la evolución temporal de los centros bajo protocolos que involucran la estimulación con luz y con radiación de microondas.

La idea central de este trabajo es que el esquema de pulso múltiple abre nuevas perspectivas en las capacidades de detección de respuesta temporal basadas únicamente en el mecanismo de recombinación dependiente de espín en ausencia de un campo magnético externo.

Aunque aquí nos hemos enfocado en los centros $\mathrm{Ga}^{2+}$ del GaAsN, este método pude fácilmente ser adaptado a otros tipos de defectos puntuales con propiedades comparables a los $\mathrm{Ga}^{2+}$ incorporados en diferentes semiconductores, teniendo en cuenta que los centros sean capaces de capturar selectivamente a los electrones de la banda de conducción a partir de su orientación de espín. La dinámica del espín mostrada por la técnica de pulso-prueba desarrollada aquí proporciona directrices generales sobre las propiedades deseadas de los sistemas de espín basados en defectos puntuales.

En el futuro, los centros que exhiben mecanismos de recombinación dependientes del espín integrados a una matriz semiconductora que no tiene espín nuclear, y en consecuencia una relajación del espín nuclear muy lenta se pueden ocupar para crear estados de momento angular estables o qubits estables. El uso del mecanismo de recombinación dependiente de espín presenta 
claras ventajas en la creación de dispositivos que conjugan la electrónica estándar con la espintrónica mediante el acoplamiento de la polarización de los electrones de la banda de conducción con los espines nucleares en los defectos puntuales. El estado del espín de dichos sistemas podría activarse y rastrearse mediante el método de doble pulso presentado aquí. 


\section{Operadores}

\section{A.1. Operadores del hamiltoniano}

Los operadores para el espín electrónico en la banda de conducción están dados por

$$
\begin{aligned}
\hat{S}_{x} & =\frac{1}{2}\left(\delta_{k, 2} \delta_{l, 3}+\delta_{k, 3} \delta_{l, 2}\right), \\
\hat{S}_{y} & =\frac{i}{2}\left(\delta_{k, 2} \delta_{l, 3}-\delta_{k, 3} \delta_{l, 2}\right), \\
\hat{S}_{z} & =\frac{1}{2}\left(-\delta_{k, 2} \delta_{l, 2}+\delta_{k, 3} \delta_{l, 3}\right) .
\end{aligned}
$$

Los operadores para el espín del electrón ligado y el espín nuclear de la trampa no apareada esta dado por $\hat{\mathbf{S}}_{c} \otimes \hat{\mathbb{I}}_{\mathbf{I}_{1}} \hat{\mathbb{I}}_{\mathbf{S c}} \otimes \hat{\mathbf{I}}_{1}$ respectivamente. Los operadores para el espín del electrón son:

$$
\begin{aligned}
\hat{S}_{c x} & =\frac{1}{2} \sum_{r=4}^{7}\left(\delta_{k, 4+r} \delta_{l, r}+\delta_{k, r} \delta_{l, 4+r}\right), \\
\hat{S}_{c y} & =\frac{i}{2} \sum_{r=4}^{7}\left(-\delta_{k, 4+r} \delta_{l, r}+\delta_{k, r} \delta_{l, 4+r}\right), \\
\hat{S}_{c z} & =\frac{1}{2} \sum_{r=4}^{7}\left(-\delta_{k, r} \delta_{l, r}+\delta_{k, 4+r} \delta_{l, 4+r}\right),
\end{aligned}
$$


y para el espín nuclear son:

$$
\begin{aligned}
\hat{I}_{1 x} & =\frac{\sqrt{3}}{2} \sum_{r=2}^{5}\left(\delta_{k, 2 r+1} \delta_{l, 2 r}+\delta_{k, 2 r} \delta_{l, 2 r+1}\right) \\
& +\sum_{r=0}^{1}\left(\delta_{k, 4 r+6} \delta_{l, 4 r+5}+\delta_{k, 4 r+5} \delta_{l, 4 r+6}\right), \\
\hat{I}_{1 y} & =i \frac{\sqrt{3}}{2} \sum_{r=2}^{5}\left(-\delta_{k, 2 r+1} \delta_{l, 2 r}+\delta_{k, 2 r} \delta_{l, 2 r+1}\right) \\
& +i \sum_{r=0}^{1}\left(-\delta_{k, 4 r+6} \delta_{l, 4 r+5}+\delta_{k, 4 r+5} \delta_{l, 4 r+6}\right), \\
\hat{I}_{1 z} & =\frac{3}{2} \sum_{r=0}^{1}\left(-\delta_{k, 4 r+4} \delta_{l, 4 r+4}+\delta_{k, 4 r+7} \delta_{l, 4 r+7}\right) \\
& +\frac{1}{2} \sum_{r=0}^{1}\left(-\delta_{k, 4 r+5} \delta_{l, 4 r+5}+\delta_{k, 4 r+6} \delta_{l, 4 r+6}\right) .
\end{aligned}
$$

Por último los operadores correspondientes a las tres componenete de espín nuclear de las trampas apareadas estan dados por

$$
\begin{aligned}
\hat{I}_{2 x} & =\frac{\sqrt{3}}{2}\left(\delta_{k, 13} \delta_{l, 12}+\delta_{k, 15} \delta_{l, 14}+\delta_{k, 12} \delta_{l, 13}+\delta_{k, 14} \delta_{l, 15}\right) \\
& +\left(\delta_{k, 14} \delta_{l, 13}+\delta_{k, 13} \delta_{l, 14}\right) \\
\hat{I}_{2 y} & =i \frac{\sqrt{3}}{2}\left(-\delta_{k, 13} \delta_{l, 12}-\delta_{k, 15} \delta_{l, 14}+\delta_{k, 12} \delta_{l, 13}+\delta_{k, 14} \delta_{l, 15}\right) \\
& +i\left(-\delta_{k, 14} \delta_{l, 13}+\delta_{k, 13} \delta_{l, 14}\right), \\
\hat{I}_{2 z} & =\frac{3}{2}\left(-\delta_{k, 12} \delta_{l, 12}+\delta_{k, 15} \delta_{l, 15}\right)+\frac{1}{2}\left(-\delta_{k, 13} \delta_{l, 13}+\delta_{k, 14} \delta_{l, 14}\right) .
\end{aligned}
$$

Donde $k, l$ toman valores de 1 a 15 . 


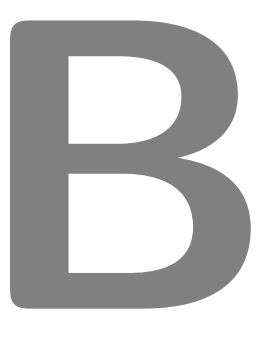

\section{Ecuación maestra para dos isótopos de $\mathbf{G a}^{2+}$}

$\mathrm{E}$ n este apéndice presentamos un modelo basado en la ecuación maestra para estudiar el comportamiento de los dos isótopos de los centros $\mathrm{Ga}^{2+}$ embebidos en el GaAsN. El ingrediente más importante del modelo es la interacción hiperfina entre los espines electrónico y nuclear de los centros $\mathrm{Ga}^{2+}$. Consideramos la interacción Zeeman en los electrones de la BC y de las trampas paramagnéticas. También tomamos en cuenta los mecanismos de relajación de espín electrónico en la BC y en las trampas; los procesos de relajación de espín nuclear debido a las interacciones de dipolo-dipolo. El sistema lo estudiamos en ausencia y presencia de un campo magnético externo en configuración de Faraday.

\section{B.1. Modelo para dos isótopos de $\mathrm{Ga}^{2+}$}

La mayoría de los procesos relevantes por la recombinación dependiente del espń en los centros de $\mathrm{Ga}^{2+}$ pueden moelarse usando la ecuación

$$
\frac{d \rho}{d t}=\frac{i}{\hbar}[\rho, H]+\mathcal{D}(\rho),
$$

donde $\rho$ es la matriz de densidad, $H$ es el hamiltoniano y $\mathcal{D}$ es el disipador. Asumimos que los distintos elementos (electrones, huecos y trampas) no comparten términos coherentes así que la matriz de densidad la expresamos como la suma directa de las submatrices

$$
\rho=\rho^{V B} \oplus \rho^{C B} \oplus \rho_{1}^{(1)} \oplus \rho_{2}^{(1)} \oplus \rho_{1}^{(2)} \oplus \rho_{2}^{(2)},
$$

donde los superíndices (1) y (2) etiquetan a los isótopos de Ga : Ga ${ }^{69}$ y Ga ${ }^{71}$.

El hamiltoniano

$$
H=\hbar \boldsymbol{\omega} \cdot \boldsymbol{S}+\boldsymbol{\Omega} \cdot\left(\boldsymbol{S}^{(1)}+\boldsymbol{S}^{(2)}\right)+\mathcal{A}_{1} \boldsymbol{J}_{1}^{(1)} \cdot \boldsymbol{S}^{(1)}+\mathcal{A}_{2} \boldsymbol{J}_{1}^{(2)} \cdot \boldsymbol{S}^{(2)},
$$


considera las interacciones de Zeeman en la $\mathrm{BC}$ y en las trampas así como la interacción hiperfina en los centros $\mathrm{Ga}^{2+}$. En la expresión anterior, $\mathcal{A}_{1}$ y $\mathcal{A}_{2}$ son los constantes hiperfinas de los isótopos $\mathrm{Ga}^{69}$ y $\mathrm{Ga}^{71}, \boldsymbol{\omega}=g \mu_{B} \eta \boldsymbol{B} / \hbar$, $\boldsymbol{\Omega}=g_{c} \mu_{B} \eta \boldsymbol{B} / \hbar, \mu_{B}$ es el magnetón de Bohr, $\boldsymbol{B}$ es el campo magnético externo, $g=+1$ y $g_{c}=+2$ son los factores giromagnéticos de los electrones en la BC y en las trampas [5, 73, 74]. El factor $\eta$ es una función de la potencia de iluminación que usamos para corregir la dependencia del campo magnético en la configuración de Faraday en las curvas de $P_{e}\left(B_{z}\right)$. Debido a los mecanismos de despolarización de espín aún no identificados, el ancho de las Lorentzianas invertidas de las curvas $P_{e}\left(B_{z}\right)$ no es reproducido correctamente por el modelo [1]. La discrepancia entre los datos experimentales y los resultados teóricos se corrige eligiendo $\eta=2.77$.

Los operadores espín $\boldsymbol{S}, \boldsymbol{S}^{(1)}$ y $\boldsymbol{S}^{(2)}$ corresponden a los electrones en la $\mathrm{BC}$, en el $\mathrm{Ga}^{69}$ y en el $\mathrm{Ga}^{71}$, respectivamente. Los operadores de espín nuclear $\boldsymbol{J}_{i}^{(1)}$ y $\boldsymbol{J}_{i}^{(2)}$ están asociados a los isótopos de $\mathrm{Ga}^{69}$ y $\mathrm{Ga}^{71}$, respectivamente. Usamos el subíndice $i=1$ para referirnos a los centros que tienen un electrón sin aparear. Para el caso de $i=2$, los centros paramagnéticos tiene dos electrones que forman un estado de singulete.

El disipador está compuesto de los siguientes elementos

$$
\begin{aligned}
\mathcal{D}(\rho)=\mathcal{G}+\mathcal{D}_{S}+\mathcal{D}_{S C}^{(1)}+\mathcal{D}_{S C}^{(2)}+\mathcal{D}_{1}^{(1)} & +\mathcal{D}_{2}^{(1)} \\
& +\mathcal{D}_{1}^{(2)}+\mathcal{D}_{2}^{(2)}+\mathcal{D}_{S D R}^{(1)}+\mathcal{D}_{S D R}^{(2)} .
\end{aligned}
$$

El término de generación esta dado por

$$
\mathcal{G}=\left(G_{+}+G_{-}\right)(p+n) 2+\left(G_{+}-G_{-}\right) \sum_{k=1}^{3} e_{k} S_{k},
$$

donde $n=S_{0}$ es el operador para los electrones en la BC y $e_{k}$ son los elementos del vector unitario de la dirección de propagación de la excitación de la luz. La generación de los electrones con espín hacia arriba y hacia abajo está dada por

$$
G(t)_{ \pm}=\frac{G_{0} P t_{p p}}{2 \sqrt{2 \pi} s_{t}}\left[\left(1 \pm P_{e 1}\right) \mathrm{e}^{-\frac{1}{2}\left(\frac{t}{s_{t}}\right)^{2}}+\alpha\left(1 \pm P_{e 2}\right) \mathrm{e}^{-\frac{1}{2}\left(\frac{t-\Delta t}{s_{t}}\right)^{2}}\right]
$$

donde $P$ es la potencia de excitación sobre un tiempo de $t_{p p}=1 \mathrm{~ns}, G_{0}=$ $3.03 \times 10^{23} \mathrm{~cm}^{-3} \mathrm{~mW}^{-1}$ es el factor de generación y $s_{t}=1$ ps en esl tiempo de duración de ambos pulsos. El grado de polarizción del pulso y del pulso prueba están parametrizados por $P_{e 1}, P_{e 2} \in[-1,1]$, respectivamente. El parámaetro $\alpha$ es la razón de las potencias entre el pulso y el pulso prueba. 
Consideramos que $\alpha=1$. El tiempo de retardo entre el pulso y el pulso prueba es $\Delta t$.

Usamos la teoría de relajación de Wangsness-Bloch-Redfield [1, 78-81] para los términos de relajación los espines electrónico y nuclear, obteniendo así los siguientes disipadores

$$
\begin{aligned}
\mathcal{D}_{S} & =-\frac{1}{2 \tau_{s}} \sum_{k=1}^{3}\left[S_{k},\left[S_{k}, \rho\right]\right], \\
\mathcal{D}_{S C}^{(i)} & =-\frac{1}{2 \tau_{s c}} \sum_{k=1}^{3}\left[S_{k}^{c},\left[S_{k}^{c}, \rho\right]\right], \\
\mathcal{D}_{q}^{(i)} & =-\frac{1}{3 \tau_{n, q}} \sum_{k=1}^{3}\left[J_{q, k}^{c},\left[J_{q, k}^{c}, \rho\right]\right] .
\end{aligned}
$$

El disipador en la ecaución (B.9) considera que los mecanismos de relajación de espín nuclear de la centros de $\mathrm{Ga}^{2+}$ están dados por las interacciones tipo dipolo-dipolo [1, 81]. Los parámetros fenomenológicos $\tau_{s}, \tau_{s c}, \tau_{n, q}$ regulan la relajación del espín electrónico y nuclear. Los mejores valores arrojados por los ajustes experimentales fueron $\tau_{s}=100 \mathrm{ps}, \tau_{s c}=200 \mathrm{~ns}, \tau_{n, 1}=1350 \mathrm{ps} \mathrm{y}$ $\tau_{n, 2}=350$ ps para los electrones en la BC, los electrones en las trampas y los nucleos en los centros [2], respectivamente. El mecanismo de la recombinación dependiente del espín está tomada en cuenta por los disipadores $\mathcal{D}_{S D R}^{(1)}$ y $\mathcal{D}_{S D R}^{(2)}$. La construcción de estos disipadores se simplifica enormemente al utilizar los elementos de un espacio interno de matrices hermitianas que describen el espacio de la matriz de densidad. El espacio interno esta constituido por los siguientes elementos

$$
\begin{aligned}
\Lambda=\left\{p, S_{k}, U_{k, j, i}^{(1)}, V_{j, i}^{(1)}, U_{k, j, i}^{(2)}, V_{j, i}^{(2)}\right\} & \\
= & \left\{\lambda_{1}, \lambda_{2}, \ldots, \lambda_{165}\right\}, \quad i, j, k=0,1,2,3
\end{aligned}
$$


donde

$$
\begin{aligned}
p= & 1_{1 \times 1} \oplus 0_{2 \times 2} \oplus 0_{8 \times 8} \oplus 0_{4 \times 4} \oplus 0_{8 \times 8} \oplus 0_{4 \times 4}, \\
S_{k}= & 0_{1 \times 1} \oplus\left(s_{k}\right)_{2 \times 2} \oplus 0_{8 \times 8} \oplus 0_{4 \times 4} \oplus 0_{8 \times 8} \oplus 0_{4 \times 4}, \\
U_{k, j, i}^{(1)}= & 0_{1 \times 1} \oplus 0_{2 \times 2} \oplus\left(s_{k} \otimes s_{j} \otimes s_{i}\right)_{8 \times 8} \\
V_{j, i}^{(1)}= & 0_{1 \times 1} \oplus 0_{4 \times 4} \oplus 0_{8 \times 8} \oplus 0_{4 \times 4}, \\
& \oplus 0_{8 \times 8} \oplus 0_{4 \times 4}, \\
U_{k, j, i}^{(2)}= & \left.0_{1 \times 1} \oplus 0_{2 \times 2} \oplus 0_{8 \times 8} \oplus 0_{4 \times 4} \otimes s_{i}\right)_{4 \times 4} \\
V_{j, i}^{(2)}= & \left.0_{1 \times 1} \oplus 0_{2 \times 2} \oplus 0_{8 \times 8} \oplus 0_{4 \times 4} \otimes s_{i}\right)_{8 \times 8} \oplus 0_{4 \times 4}, \\
& \oplus 0_{8 \times 8} \oplus\left(s_{j} \otimes s_{i}\right)_{4 \times 4} .
\end{aligned}
$$

$s_{k}$ son las matrices de espín que siguen las reglas de conmutación

$$
\left[s_{i}, s_{j}\right]=i \hbar \sum_{k} \epsilon_{i, j, k} s_{k}, \quad i, j, k=1,2,3,
$$

y $s_{0}=1_{2 \times 2}$ es la matriz identidad. La densidad de huecos en la BV está representada por el operador $p$ y $S_{k}$ con los operadores de espín de los electrones en la BC. Las operadores de espín electrónico para los isótopos $\mathrm{Ga}^{69}$ $(l=1)$ and $\mathrm{Ga}^{71}(l=2)$ están dados por

$$
S_{k}^{(l)}=U_{k, 0,0}^{(l)}, \quad k=1,2,3 .
$$

El número de centros no apareados y apareados son

$$
\begin{aligned}
& N_{1}^{(l)}=U_{0,0,0}^{(l)}, \\
& N_{2}^{(l)}=V_{0,0}^{(l)} .
\end{aligned}
$$

Los elementos de $\Lambda$ forman un álgebra de Lie finita y sus elementos son ortogonales. La ortogonalidad de estos elementos esta dado por la traza del producto de dos elementos

$$
\left(\lambda_{q}, \lambda_{q^{\prime}}\right)=\operatorname{tr}\left[\lambda_{q}^{\dagger} \lambda_{q^{\prime}}\right]=\operatorname{Tr}\left[\lambda_{q}^{2}\right] \delta_{q, q^{\prime}} .
$$

Usando estas propiedades, podemos expresar los operadores de espín nuclear fácilmente como la superposición de los elementos de la base B.10 por

$$
\begin{aligned}
J_{1, k}^{(l)} & =\sum_{j, i=0}^{3} \frac{\operatorname{Tr}\left[J_{1, k}^{(l)} U_{0, j, i}^{(l)}\right]}{\operatorname{Tr}\left[U_{0, j, i}^{(1)} U_{0, j, i}^{(1)}\right]}, \\
J_{2, k}^{(l)} & =\sum_{j, i=0}^{3} \frac{\operatorname{Tr}\left[J_{2, k}^{(l)} V_{j, i}^{(l)}\right]}{\operatorname{Tr}\left[V_{j, i}^{(1)} V_{j, i}^{(1)}\right]} .
\end{aligned}
$$


Los disipadores $\mathcal{D}_{S D R}^{(1)}$ y $\mathcal{D}_{S D R}^{(2)}$ proyectados en la base $\Lambda$ están dados por

$$
\mathcal{D}_{S D R}^{(l)}=\sum_{q} \lambda_{q} \frac{C^{(l)}\left[\lambda_{q}\right]}{\operatorname{Tr}\left[\lambda_{q}^{2}\right]},
$$

donde $C^{(l)}\left[\lambda_{q}\right]=\operatorname{Tr}\left[\mathcal{D}_{S D R}^{(l)} \lambda_{q}\right]$. Siguiendo el procedimiento desarrollado por [1, 3] extraemos los coeficientes $C^{(1)}\left[\lambda_{q}\right.$ ] del modelo de dos cargas [4, 5] y los generalizamo con el fin de complementar los requerimientos básicos como la isotropía del espacio y las usuales reglas de transformación de los tensores. Entonces los coeficientes son

$$
\begin{aligned}
C^{(l)}[p] & =-\gamma_{h} p F_{0,0}^{(l)}+G, \\
C^{(l)}\left[S_{0}\right] & =-\frac{\gamma_{e}}{2}\left(n U_{0,0,0}^{(l)}-4 \sum_{k=1}^{3} S_{k}^{n} U_{k, 0,0}^{(l)}\right)+G, \\
C^{(l)}\left[S_{k}\right] & =\frac{\gamma_{e}}{2}\left(n U_{r, 0,0}^{(l)}-S_{r}^{n} U_{0,0,0}^{(l)}\right)+G_{s}, \\
C^{(l)}\left[U_{0, j, i}^{(l)}\right] & =-\frac{\gamma_{e}}{2}\left(n U_{0, j, i}^{(l)}-4 \sum_{r=1}^{3} S_{r}^{n} U_{r, j, i}^{(l)}\right)+\gamma_{h} p F_{j, i}, \\
C^{(l)}\left[U_{k, j, i}^{(l)}\right] & =-\frac{\gamma_{e}}{2}\left(n U_{k, j, i}^{(l)}-S_{k}^{n} U_{0, j, i}^{(l)}\right), \\
C^{(l)}\left[F_{j, i}^{(l)}\right] & =\frac{\gamma_{e}}{2}\left(n U_{0, j, i}^{(l)}-4 \sum_{r=1}^{3} S_{r}^{n} U_{r, j, i}^{(l)}\right)-\gamma_{h} p F_{j, i}^{(l)},
\end{aligned}
$$

donde $l=1,2, k=1,2,3$ y $i, j=0,1,2,3$. Las barras en los parámetros indican los promedios mecano-estadísticos dados por

$$
\lambda_{q}=\operatorname{Tr}\left[\hat{\rho}(t) \hat{\lambda}_{q}\right]
$$

Esta ecuación también impica que la matriz de densidad puede ser desarrollada como

$$
\hat{\rho}(t)=\sum_{q} \frac{1}{\operatorname{Tr}\left[\lambda_{q}^{2}\right]} \lambda_{q}(t) \hat{\lambda}_{q} .
$$

Los parámtros relacionados con las capturas de los electrones de la $\mathrm{BC}$ y los huecos en la BV están dados por $c_{n}=1 / N^{(l)} \tau^{*}$ and $c_{p}=1 / N^{(l)} \tau_{h}$ donde $N^{(1)}$ y $N^{(2)}$ son el número total isótopos de $\mathrm{Ga}^{69}$ y $\mathrm{Ga}^{71}$, respectivamente. $\tau^{*}=14.2$ ps y $\tau_{h}=12$ ps son los tiempos de recombinación de los electrones en la BC y de los huecos en la BV, respectivamente.

Para extraer información del modelo, encotramos el sistema de ecuaciones diferenciales para los valores mecanoestadísticos de los elementos de $\Lambda$. 
Obtenemos un sistema de 165 ecuaciones diferenciales no lineales de primer orden dado por

$$
\frac{d \lambda_{q}}{d t}=\frac{i}{\hbar} \operatorname{Tr}\left[\left[H, \lambda_{q}\right] \rho\right]+\operatorname{Tr}\left[\mathcal{D}(\rho) \lambda_{q}\right]
$$

La dependencia temporal de las observables físicas las obtenemos mediante

$$
\hat{O}=\operatorname{Tr}[\hat{O} \hat{\rho}(t)]=\sum_{q} \frac{1}{\operatorname{Tr}\left[\lambda_{q}^{2}\right]} \operatorname{Tr}\left[\hat{O} \hat{\lambda}_{q}\right] \lambda_{q}(t)
$$

donde $\hat{O}$ es cualquier operador desarrollado en la base $\Lambda$.

En particular, la razón de recombinación dependiente del espín $S D R_{r}$ fue calculada como $S D R_{r}=I_{+} / I_{X}$ donde $I_{+}$y $I_{X}$ son las intensidades de fotoluminiscencia bajo excitación circular y linear, respectivamente. Las intensidades de fotolumniscencia fueron calculadas como

$$
I=\gamma_{r} n(t) p(t)
$$

donde $n(t)=\operatorname{Tr}\left[\hat{\rho}(t) \hat{S}_{0}\right], p(t)=\operatorname{Tr}[\hat{\rho}(t) \hat{p}]$ y $\gamma_{r}=3 c_{p} / 20$ es el coeficiente de recombinación bimolecular. Para obtener $I_{+}$el cálculo fue desarrollado con $P_{e 2}=0.15$ y para $J_{X}$ consideramos que $P_{e 2}=0$. 


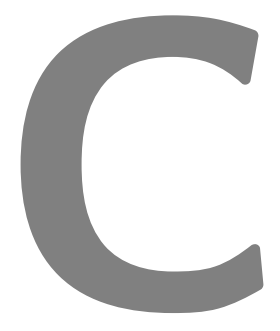

\section{Artículos publicados}

n este capítulo presento los ocho artículos en los que participé durante mi estancia en el doctorado. Los primeros cuatro artículos sustentan a la tesis doctoral. Los restantes cuatro son trabajos en los que colaboré sobre temas afines al tema de doctorado

Los artículos que sustentan a la tesis doctoral son

- Time evolution of two-dimensional quadratic hamiltonians: A lie algebraic approach [54].

- Electron-nuclear spin dynamics of $\mathrm{Ga}^{2+}$ paramagnetic centers probed by spin-dependent recombination: A masterequation approach [1].

- Electron-nuclear coherent spin oscillations probed by spin-dependent re-combination [2].

- Electron-nuclear spin dynamics of ga centers in gaasn dilute ni-tride semiconductors probed by pump-probe spectroscopy [3] . 


\title{
Time evolution of two-dimensional quadratic Hamiltonians: A Lie algebraic approach
}

\author{
J. C. Sandoval-Santana, ${ }^{1}$ V. G. Ibarra-Sierra, ${ }^{1}$ J. L. Cardoso, ${ }^{2}$ \\ and A. Kunold ${ }^{2}$ \\ ${ }^{1}$ Departamento de Física, Universidad Autónoma Metropolitana Iztapalapa, Av. San Rafael \\ Atlixco 186, Col. Vicentina, 09340 México D.F., Mexico \\ ${ }^{2}$ Área de Física Teórica y Materia Condensada, Universidad Autónoma Metropolitana \\ Azcapotzalco, Av. San Pablo 180, Col. Reynosa-Tamaulipas, Azcapotzalco, \\ 02200 México D.F., México
}

(Received 2 February 2016; accepted 11 April 2016; published online 26 April 2016)

\begin{abstract}
We develop a Lie algebraic approach to systematically calculate the evolution operator of a system described by a generalized two-dimensional quadratic Hamiltonian with time-dependent coefficients. Although the development of the Lie algebraic approach presented here is mainly motivated by the two-dimensional quadratic Hamiltonian, it may be applied to investigate the evolution operators of any Hamiltonian having a dynamical algebra with a large number of elements. We illustrate the method by finding the propagator and the Heisenberg picture position and momentum operators for a two-dimensional charge subject to uniform and constant electromagnetic fields. Published by AIP Publishing. [http://dx.doi.org/10.1063/1.4947296]
\end{abstract}

\section{INTRODUCTION}

In many applications as radio-frequency ion traps, ${ }^{1-8}$ quantum optics, ${ }^{9-12}$ cosmology, ${ }^{13,14}$ quantum field theory, ${ }^{15}$ quantum dissipation, ${ }^{16-22}$ magneto-transport in lateral heterostructures ${ }^{23-26}$ and even gravitational waves ${ }^{27}$ the time evolution of particles in quadratic potentials is frequently examined. The one-dimensional, generalized time-dependent quadratic Hamiltonian is given by

$$
\hat{H}=a_{1}(t)+a_{2}(t) \hat{x}+a_{3}(t) \hat{p}+a_{4}(t) \hat{x}^{2}+a_{5}(t) \hat{p}^{2}+a_{6}(t)(\hat{x} \hat{p}+\hat{p} \hat{x}),
$$

where $\hat{x}$ and $\hat{p}$ are the position and momentum operators following the standard commutation relations. Aside from the simple harmonic oscillator, a large number of interesting systems arise from this Hamiltonian as the linear potential, ${ }^{28,29}$ the driven harmonic oscillator, ${ }^{30,31}$ Kanai-Caldirola Hamiltonians, ${ }^{16-21}$ and time dependent harmonic oscillators, i.e., an oscillator with time-varying frequency. ${ }^{21,32,33}$ The time evolution generated by the most general version of (1) has been studied by means of Lewis and Riesenfeld ${ }^{34}$ invariants $^{35}$ and through linear invariants. ${ }^{1}$

Combining two one-dimensional generalized quadratic Hamiltonians along the $x$ and $y$ coordinates and adding cross terms for the position and momentum operators one arrives at the most general form of the two-dimensional quadratic Hamiltonian

$$
\begin{array}{r}
\hat{H}=a_{1}(t)+a_{2}(t) \hat{x}+a_{3}(t) \hat{y}+a_{4}(t) \hat{p}_{x}+a_{5}(t) \hat{p}_{y}+a_{6}(t) \hat{x}^{2}+a_{7}(t) \hat{y}^{2}+a_{8}(t) \hat{x} \hat{y}+a_{9}(t) \hat{p}_{x}^{2} \\
+a_{10}(t) \hat{p}_{y}^{2}+a_{11}(t) \hat{p}_{x} \hat{p}_{y}+a_{12}(t)\left(\hat{x} \hat{p}_{x}+\hat{p}_{x} \hat{x}\right)+a_{13}(t)\left(\hat{y} \hat{p}_{y}+\hat{p}_{y} \hat{y}\right) \\
+a_{14}(t) \hat{x} \hat{p}_{y}+a_{15}(t) \hat{y} \hat{p}_{x}
\end{array}
$$

Many Hamiltonians of physical significance stem from (2), for example, a single electron in an elliptically shaped quantum dot with quadratic confining potential, a charge subject to variable electromagnetic fields or two-dimensional quadratic Kanai-Caldirola Hamiltonians among others.

Some special cases emerging from these Hamiltonians have been studied by diverse mathematical methods. For instance, the time dependent linear potential has been treated through the Lewis and Riesenfeld ${ }^{34}$ invariant theory, ${ }^{28,29,36}$ Feynman's path integrals, ${ }^{37-41}$ time-space transformation 


\title{
Electron-nuclear spin dynamics of $\mathrm{Ga}^{2+}$ paramagnetic centers probed by spin-dependent recombination: A master equation approach
}

\author{
V. G. Ibarra-Sierra, ${ }^{1}$ J. C. Sandoval-Santana, ${ }^{1}$ S. Azaizia, ${ }^{2}$ H. Carrère, ${ }^{2}$ L. A. Bakaleinikov, ${ }^{3}$ V. K. Kalevich, ${ }^{3}$ \\ E. L. Ivchenko, ${ }^{3}$ X. Marie, ${ }^{2}$ T. Amand, ${ }^{2}$ A. Balocchi, ${ }^{2}$ and A. Kunold ${ }^{4}$ \\ ${ }^{1}$ Departamento de Física, Universidad Autónoma Metropolitana Iztapalapa, Av. San Rafael Atlixco 186, \\ Col. Vicentina, 09340 Cuidad de México, Mexico \\ ${ }^{2}$ Universitè de Toulouse, INSA-CNRS-UPS, LPCNO, 135 avenue de Rangueil, 31077 Toulouse, France \\ ${ }^{3}$ Ioffe Physical-Technical Institute, 194021 St. Petersburg, Russia \\ ${ }^{4}$ Área de Física Teórica y Materia Condensada, Universidad Autónoma Metropolitana Azcapotzalco, \\ Av. San Pablo 180, Col. Reynosa-Tamaulipas, 02200 Cuidad de México, Mexico \\ (Received 13 November 2016; revised manuscript received 3 April 2017; published 10 May 2017)
}

\begin{abstract}
Similar to nitrogen-vacancy centers in diamond and impurity atoms in silicon, interstitial gallium deep paramagnetic centers in GaAsN have been proven to have useful characteristics for the development of spintronic devices. Among other interesting properties, under circularly polarized light, gallium centers act as spin filters that dynamically polarize free and bound electrons reaching record spin polarizations (close to 100\%). Furthermore, the recent observation of the amplification of the spin filtering effect under a Faraday configuration magnetic field has suggested that the hyperfine interaction that couples bound electrons and nuclei permits the optical manipulation of the nuclear spin polarization. Even though the mechanisms behind the nuclear spin polarization in gallium centers are fairly well understood, the origin of nuclear spin relaxation and the formation of an Overhauser-like magnetic field remain elusive. In this work we develop a model based on the master equation approach to describe the evolution of electronic and nuclear spin polarizations of gallium centers interacting with free electrons and holes. Our results are in good agreement with existing experimental observations. In particular, we are able to reproduce the amplification of the spin filtering effect under a circularly polarized excitation in a Faraday configuration magnetic field. In regard to the nuclear spin relaxation, the roles of nuclear dipolar and quadrupolar interactions are discussed. Our findings show that, besides the hyperfine interaction, the spin relaxation mechanisms are key to understand the amplification of the spin filtering effect and the appearance of the Overhauser-like magnetic field. To gain a deeper insight in the interplay of the hyperfine interaction and the relaxation mechanisms, we have also performed calculations in the pulsed excitation regime. Our model's results allow us to propose an experimental protocol based on time-resolved spectroscopy. It consists of a pump-probe photoluminescence scheme with the detection and the tracing of the electron-nucleus flip-flops through photoluminescence measurements.
\end{abstract}

DOI: 10.1103/PhysRevB.95.195204

\section{INTRODUCTION}

Negatively charged nitrogen-vacancy centers in diamond [1-5], phosphorous atom impurities in silicon [6-11], and other schemes based on point defects embedded in semiconductors have been widely studied as alternatives to develop quantum bits $[12,13]$. One of the necessary conditions for quantum computing is long electron spin decoherence times to ensure a minimum of fault tolerance [10,14]. In diamond's nitrogen-vacancy centers [15], silicon vacancies in silicon carbide [16], silicon [17], and any III-V based quantum dots $[10,18]$ the fluctuating nuclear bath is the main source limiting spin coherence time. The nuclear dipole-dipole interaction is believed to be the dominant mechanism behind the diffusion-induced electron-spin decoherence [19]. To protect the dynamics of the nuclear spins of point defects from the decoherence induced by the environment, semiconductors mainly composed of spin-zero isotopes such as silicon and carbon are preferred over III-V semiconductors [5]. Even though the two stable isotopes of $\mathrm{Ga},{ }^{69} \mathrm{Ga}$ and ${ }^{71} \mathrm{Ga}$, have nuclear spin $3 / 2$, in dilute nitride $\mathrm{GaAsN}$ point interstitial defects give rise to paramagnetic centers that have very peculiar and useful properties. One of them is the spin-dependent recombination (SDR) [20-27]. In Ga(In)NAs alloys, $\mathrm{Ga}_{i}^{2+}$ paramagnetic centers with only one bound electron can selectively capture another conduction band (CB) electron with the opposite spin orientation [26-30]. Due to this mechanism, paramagnetic centers act as a spin filter that blocks the recombination of $\mathrm{CB}$ electrons with the same spin and efficiently captures electrons whose spin is in the opposite direction. In the centers, the bound and captured electrons form a singlet state that is destroyed as either one of the electrons recombines to the valence band (VB). It is important to note that while the lifetime of conduction electrons with the spin opposite to that of the bound electrons is a few picoseconds, the lifetime of conduction electrons copolarized with the majority of bound electrons may extend to nanoseconds. As a consequence the free photoelectron spin polarization can reach over $80 \%$ under circularly polarized incident light. Additionally the photoluminescence (PL) intensity can be as high as $800 \%$ under circularly polarized optical excitation compared to a linearly polarized one [21,22]. The increase in CB electron population allows even for the detection of electron spin polarization by electrical means due to a giant photoconductivity effect under circularly polarized light $[24,31]$.

Whereas in diamond and silicon the optical excitation acts directly on the point defects, in GaAsN the bound electron is 


\title{
Electron-nuclear coherent spin oscillations probed by spin-dependent recombination
}

\author{
S. Azaizia, ${ }^{1}$ H. Carrère, ${ }^{1}$ J. C. Sandoval-Santana, ${ }^{2}$ V. G. Ibarra-Sierra, ${ }^{2}$ V. K. Kalevich, ${ }^{3}$ E. L. Ivchenko, ${ }^{3}$ L. A. Bakaleinikov, ${ }^{3}$ \\ X. Marie, ${ }^{1}$ T. Amand, ${ }^{1}$ A. Kunold ${ }^{4}$ and A. Balocchi ${ }^{1, *}$ \\ ${ }^{1}$ Universitè de Toulouse, INSA-CNRS-UPS, LPCNO, 135 avenue de Rangueil, 31077 Toulouse, France \\ ${ }^{2}$ Departamento de Física, Universidad Autónoma Metropolitana Iztapalapa, Avenida San Rafael Atlixco 186, \\ Colonia Vicentina, 09340 Mexico City, Mexico \\ ${ }^{3}$ Ioffe Physical-Technical Institute, 194021 St. Petersburg, Russia \\ ${ }^{4}$ Área de Física Teórica y Materia Condensada, Universidad Autónoma Metropolitana Azcapotzalco, Avenida San Pablo 180, Colonia \\ Reynosa-Tamaulipas, 02200 Mexico City, Mexico
}

(Received 1 March 2018; published 5 April 2018)

\begin{abstract}
We demonstrate the triggering and detection of coherent electron-nuclear spin oscillations related to the hyperfine interaction in Ga deep paramagnetic centers in $\mathrm{GaAsN}$ by band-to-band photoluminescence without an external magnetic field. In contrast to other point defects such as $\mathrm{Cr}^{4+}$ in $\mathrm{SiC}, \mathrm{Ce}^{3+}$ in yttrium aluminum garnet crystals, nitrogen-vacancy centers in diamond, and $\mathrm{P}$ atoms in silicon, the bound-electron spin in Ga centers is not directly coupled to the electromagnetic field via the spin-orbit interaction. However, this apparent drawback can be turned into an advantage by exploiting the spin-selective capture of conduction band electrons to the $\mathrm{Ga}$ centers. On the basis of a pump-probe photoluminescence experiment we measure directly in the temporal domain the hyperfine constant of an electron coupled to a gallium defect in GaAsN by tracing the dynamical behavior of the conduction electron spin-dependent recombination to the defect site. The hyperfine constants and the relative abundance of the nuclei isotopes involved can be determined without the need of an electron spin resonance technique and in the absence of any magnetic field. Information on the nuclear and electron spin relaxation damping parameters can also be estimated from the oscillation amplitude decay and the long-time-delay behavior.
\end{abstract}

DOI: 10.1103/PhysRevB.97.155201

\section{INTRODUCTION}

Electron and nuclear spins of well-isolated point defects in semiconductors are excellent candidates for understanding fundamental spin-coupling mechanisms and are a model system for quantum information processing. The coupling through hyperfine interaction (HFI) represents a key spin mechanism in semiconductor systems: responsible for creating mixed electron-nuclear spin states, it has been shown to be useful, e.g., for electron-nuclear spin transfer, in controlling the electron spin coherence time of $\mathrm{P}$ donor sites in Si [1-6], and the nitrogen-vacancy centers in diamond [7-16]. The HFI is, however, also responsible for electron and nuclear spin relaxation and decoherence [17].

Similar to nitrogen in diamond, on the one hand, and to shallow defects in silicon, on the other hand, interstitial $\mathrm{Ga}_{i}^{2+}$ defects in the dilute nitride GaAsN $[18,19]$ unite the characteristics of deep and well-isolated paramagnetic centers with an electrically and optically addressable semiconducting system, leading, e.g., to the giant spin-dependent photoconductivity effect $[20,21]$. The incorporation of nitrogen in (In)GaAs gives rise to paramagnetic interstitial centers composed of a $\mathrm{Ga}_{i}^{2+}$ atom and a single resident electron [19]. These defect sites are at the origin of a very efficient spin-dependent recombination of conduction-band (CB) electrons. This has proven to be an effective tool for, for instance, generating an exceptionally high spin polarization (up to $\sim 100 \%$ ) of free and bound electrons

\footnotetext{
${ }^{*}$ Corresponding author: andrea.balocchi@insa-toulouse.fr
}

in these nonmagnetic dilute nitride semiconductors at room temperature [22-25].

Optically or electrically detected magnetic resonance techniques are consistently employed for manipulating and probing defect spins through the hyperfine interaction and also to identify the defect chemical nature [26,27]. Optically detected magnetic resonance, a variation of nuclear resonance techniques, has been demonstrated on $\mathrm{Cr}^{4+}$ impurity ensembles in $\mathrm{SiC}$ and $\mathrm{GaN}$ [28]. Mostly due to the weak magnetization of nuclear spins, these methods have very low sensitivity. Furthermore, attempts to improve the detection involve high magnetic fields and cryogenic temperatures. Time-resolved Faraday rotation has been successfully used to monitor the $5 d$ electron spin time evolution of $\mathrm{Ce}^{3+}$ point defects in yttrium aluminum garnet crystals [29]. These techniques partly rely on the spin polarization of electrons bound to centers by coupling the spin states and the electromagnetic field via the spin-orbit interaction occurring in orbitals other than $s$. Despite the many similarities, in this regard, $\mathrm{Ga}_{i}^{2+}$ centers are fundamentally different. The $4 s$ electron spin in $\mathrm{Ga}_{i}^{2+}$ point defects, unlike the $5 d$ electron spin in $\mathrm{Ce}^{3+}$, is not directly coupled to the electromagnetic field. However, in these centers $\mathrm{CB}$ electrons recombine according to their spin orientation, dynamically polarizing the bound-electron spins. This spindependent recombination process, which basically relies on the Pauli exclusion principle, not only can be used to spin polarize the bound electron and to control the degree of nuclear spin polarization [30] but can also be exploited to sense the electron spin dynamics with a time resolution of about $10 \mathrm{ps}$. 


\title{
Electron-nuclear spin dynamics of Ga centers in GaAsN dilute nitride semiconductors probed by pump-probe spectroscopy
}

\author{
J.C. Sandoval-Santana ${ }^{1, a}$, V.G. Ibarra-Sierra ${ }^{1}$, S. Azaizia ${ }^{2}$, H. Carrère ${ }^{2}$, L.A. Bakaleinikov ${ }^{3}$, V.K. Kalevich ${ }^{3}$, \\ E.L. Ivchenko ${ }^{3}$, X. Marie ${ }^{2}$, T. Amand ${ }^{2}$, A. Balocchi ${ }^{2}$, and A. Kunold ${ }^{4}$ \\ ${ }^{1}$ Departamento de Física, Universidad Autónoma Metropolitana Iztapalapa, Av. San Rafael Atlixco 186, Col. Vicentina, 09340 \\ Cuidad de México, Mexico \\ 2 Université de Toulouse, INSA-CNRS-UPS, LPCNO, 135 avenue de Rangueil, 31077 Toulouse, France \\ 3 Ioffe Physical-Technical Institute, 194021 St. Petersburg, Russia \\ 4 Área de Física Teórica y Materia Condensada, Universidad Autónoma Metropolitana Azcapotzalco, Av. San Pablo 180, Col. \\ Reynosa-Tamaulipas, 02200 Cuidad de México, Mexico
}

Received: 14 February 2018

Published online: 26 March 2018 - (C) Società Italiana di Fisica / Springer-Verlag 2018

\begin{abstract}
We propose an experimental procedure to track the evolution of electronic and nuclear spins in $\mathrm{Ga}^{2+}$ centers in GaAsN dilute semiconductors. The method is based on a pump-probe scheme that enables to monitor the time evolution of the three components of the electronic and nuclear spin variables. In contrast to other characterization methods, as nuclear magnetic resonance, this one only needs moderate magnetic fields $(B \approx 10 \mathrm{mT})$, and does not require microwave irradiation. Specifically, we carry out a series of tests for different experimental conditions in order to optimize the procedure for maximum sensitivity in the measurement of the circular degree of polarization. Based on previous experimental results and the theoretical calculations presented here, we estimate that the method could yield a time resolution of about $10 \mathrm{ps}$.
\end{abstract}

\section{Introduction}

The prospect of novel spintronic devices and quantum computation has boosted the developement of new and powerful experimental tools to study and characterize solid-state-based spin systems. Some of the most promising proposals to implement quantum registers are nuclear-spin-based architectures [1]. In this type of configuration, a bound electron spin couples to the nuclear spin in a point defect through hyperfine interaction. Owing to their remarkable degree of isolation, nuclear spins are able to interact with the environment and the electromagnetic field mostly through the bound electron [2]. Even though this fact produces very stable quantum bits, addressing the nuclear states is rather challenging. A wide range of point defects and embedding materials has been tested. Some of them are point defects in diamond $[2,3]$, diamond negatively charged nitrogen-vacancy centers [4-9], phosphorous atom impurities in silicon [1015], silicon vacancies in silicon carbide [16] and more recently, Ge-vacancy centers embedded in diamond [17-19].

Nuclear-magnetic-resonance-based techniques provide with the means to both control the nuclear states [3] and probe the main nuclear-polarization mechanisms [20-25]. However, these techniques have very low detection sensitivity mainly caused by the weak magnetization of nuclear spins [26]. Moreover, the methods developed to enhance their sensitivity generally involve cryogenic temperatures and high magnetic fields.

Alternative methods enable time resolution and higher sensitivity. Time-resolved Faraday rotation has been used to study the evolution of spin systems, such as magnetic-semiconductor quantum wells [27], $\mathrm{Ce}^{3+}$ ions in YAG crystals [28, 29], spin precession in orthoferrites [30] and spin coherence in colloidal CdS quantum dots [31]. Other pump-probe schemes are capable of time-resolving the fast spin dynamics that take place in antiferromagnetic materials by detecting the birefringence changes induced by the pump pulse [32]. The combination of spin-polarized scanning tunneling microscopy together with an all electronic pump-probe scheme can also be applied to the observation of spin relaxation of individual atoms [33].

\footnotetext{
${ }^{a}$ e-mail: jcss@xanum.uam.mx
} 
Otros trabajos en los que colaboré durante el doctorado fueron

- Lie algebraic approach to the time-dependent quantum general harmonic oscillator and the bi-dimensional charged particle in time-dependent electromagnetic fields. Annals of Physics 362, 83-117 84

- Singularities and internal rotational dynamics of electron beams [85]

- Spin-filtering effect in GaAsN: electron-nuclear spin dynamics of $\mathrm{Ga}^{3+}$ centers 86

- Method for finding the exact effective Hamiltonian of time driven quantum systems [87] 


\title{
Lie algebraic approach to the time-dependent quantum general harmonic oscillator and the bi-dimensional charged particle in time-dependent electromagnetic fields
}

\author{
V.G. Ibarra-Sierra ${ }^{a}$, J.C. Sandoval-Santana ${ }^{a}$, J.L. Cardoso ${ }^{\text {b }}$, \\ A. Kunold ${ }^{\text {b,* }}$ \\ a Departamento de Física, Universidad Autónoma Metropolitana Iztapalapa, Av. San Rafael Atlixco 186, \\ Col. Vicentina, 09340 México D.F., Mexico \\ b Área de Física Teórica y Materia Condensada, Universidad Autónoma Metropolitana Azcapotzalco, \\ Av. San Pablo 180, Col. Reynosa-Tamaulipas, Azcapotzalco, 02200 México D.F., Mexico
}

\section{H I G H L I G H T S}

- We deal with the general quadratic Hamiltonian and a particle in electromagnetic fields.

- The evolution operator is worked out through the Lie algebraic approach.

- We also obtain the propagator and Heisenberg picture position and momentum operators.

- Analytical expressions for a rotating quadrupole field ion trap are presented.

- Exact solutions for magneto-transport in variable electromagnetic fields are shown.

\section{A R T I C L E I N F O}

Article history:

Received 17 April 2015

Accepted 23 July 2015

Available online 30 July 2015

\section{Keywords:}

Quantum harmonic oscillator

Charged particle in electromagnetic fields

Lie algebra

Evolution operator

Ion trap

\begin{abstract}
A B S T R A C T
We discuss the one-dimensional, time-dependent general quadratic Hamiltonian and the bi-dimensional charged particle in time-dependent electromagnetic fields through the Lie algebraic approach. Such method consists in finding a set of generators that form a closed Lie algebra in terms of which it is possible to express a quantum Hamiltonian and therefore the evolution operator. The evolution operator is then the starting point to obtain the propagator as well as the explicit form of the Heisenberg picture position and momentum operators. First, the set of generators forming a closed Lie algebra is identified for the general quadratic
\end{abstract}

\footnotetext{
* Corresponding author.

E-mail address: akb@correo.azc.uam.mx (A. Kunold).

http://dx.doi.org/10.1016/j.aop.2015.07.022

0003-4916/C 2015 Elsevier Inc. All rights reserved.
} 


\title{
Singularities and internal rotational dynamics of electron beams
}

\author{
D. Velasco-Martínez, ${ }^{1}$ V. G. Ibarra-Sierra, ${ }^{2}$ J. C. Sandoval-Santana, ${ }^{2}$ J. L. Cardoso, ${ }^{1}$ and A. Kunold ${ }^{1}$ \\ ${ }^{1}$ Área de Física Teórica y Materia Condensada, Universidad Autónoma Metropolitana Azcapotzalco, Avenida San Pablo 180, \\ Col. Reynosa-Tamaulipas, Azcapotzalco, 02200 Cuidad de México, México \\ ${ }^{2}$ Departamento de Física, Universidad Autónoma Metropolitana Iztapalapa, Avenida San Rafael Atlixco 186, \\ Col. Vicentina, Iztapalapa, 09340 Cuidad de México, México \\ (Received 1 August 2016; published 6 December 2016)
}

\begin{abstract}
We study the internal rotational dynamics of electronic beams in relation to the phase singularities of their wave functions. Given their complex singularity structure, Hermite-Gaussian beams and other superpositions of Laguerre-Gaussian modes are studied here. We show that by inspecting the lowest nonvanishing terms of the wave function near the singularity, it is possible to infer the structure of the Bohmian streamlines. Conversely, starting from a map of the electron's Bohmian velocities, we demonstrate that it is possible to derive the form of the electron's wave function near the singularity. We outline a procedure that could yield an experimental method to determine the main parameters of the electron's wave function close to a singularity.
\end{abstract}

DOI: 10.1103/PhysRevA.94.063815

\section{INTRODUCTION}

The charged particle subject to a uniform and static magnetic field [1-3] is at the heart of many physical theories. The quantum Hall [4] and related effects such as de Haas-van Alphen effect [5], Shubnikov-de Haas oscillations [6], and the fractional quantum Hall effect [7] cannot be understood without it. However, solid-state systems are too complex to provide precise information about the internal dynamics of the Landau levels. Inspired by optical vortex beams, electron vortex beams have recently gained attention [8-16] not only because they are expected to provide new capabilities to electron microscopes, but also for allowing the observation of the electrons' isolated quantum states. The concept of vortex beam has even been extended to two-level atom beams [17].

Whereas electron vortex beams may be understood as solutions of the Schrödinger equation, their optical counterparts are paraxial solutions of the Helmholtz equation [11-13]. In contrast to solid-state systems, Laguerre-Gaussian (LG) beams allow for more precise and accurate observation of the internal dynamics of the Landau levels because they are generated in the rather controlled environment of a transmission electron microscope (TEM).

Among other techniques, electron vortex beams with a helical wave front carrying a large orbital angular momentum (as LG beams) are produced passing a standard electron beam through a holographic mask in TEMs [11-13,18]. For example, LG beams are created by using a diffraction grating with a dislocation [13] that confers electrons a certain orbital angular momentum (OAM). In order to get actual Fock-Darwin states, the beam waist is chosen to match their diameter in a region where the axial magnetic field is uniform. When LG beams propagate along the direction of a uniform magnetic field, they behave as stable solutions of the Schrödinger equation. LG beams correspond in fact to the Fock-Darwin states that in turn are stationary solutions of the Shrödinger equation of a two-dimensional (2D) charged particle in a magnetic field. Thereby most of their features remain constant and, as for their optical counterparts, the beam profile is maintained as electrons propagate. Among these quantities, the OAM is preserved.
Bessel electron beams [19], Airy beams [20], and HermiteGaussian (HG) beams [21] have been studied and produced in the laboratory. HG modes, for example, may serve to probe the OAM of electron beams. All of these beams can be understood as linear combinations of LG states carrying different amounts of OAM.

Phase singularities play a crucial role in the formation of vortices in light. They have been widely studied since the pioneering works of Dirac [22], Aharonov and Bohm [23], Nye [24], and Berry [25]. Mathematically, optical singularities are regions in the domain of the electromagnetic field where the phase is indeterminate; as a consequence the field vanishes in these regions of space. Phase singularities are also present in electron beams and manifest themselves as vortices of density currents that turn around a zero of the wave function. Due to their cylindrical symmetry, LG beams can be made to have large OAM. Their wave front spirals around a phase singularity located at the center of the wave front [26,27]. Singularities may be characterized by their topological charge, i.e., an integer number that counts the number of times the wave front makes a full revolution in one wavelength. In the case of LG beams and other eigenstates of the OAM, the topological charge matches the OAM. Some linear combinations of LG modes are very interesting in this regard because, not carrying a distinctive angular momentum, they may present rich and complex singularity arrangements. Around each singularity, a density current vortex is formed whose properties fully depend on the mathematical form of the singularity.

Given the large amount of knowledge on optical vortices, they were the first candidates for OAM-based spectroscopic techniques. However, optical techniques have been proven to be very inefficient, owing to the weak optical multipolar transitions [28]. In contrast, it has been theoretically [29,30] and experimentally [15] demonstrated that the OAM of electron beams can be transferred to atomic electrons having an observable effect on the magnetic circular dichroism. It has also been suggested that by creating different superpositions of LG beams [31], it is possible to tailor the internal electron currents. For instance, by superimposing various LG states, it is possible to produce off-axis density current vortices [19]. 


\title{
Spin-filtering effect in GaAsN: electron-nuclear spin dynamics of $\mathrm{Ga}^{3+}$ centers
}

\author{
V. G. Ibarra-Sierra ${ }^{1}$ - J. C. Sandoval-Santana ${ }^{1}$ S S. Azaizia ${ }^{2}$ - H. Carrère ${ }^{2}$ - L. A. Bakaleinikov ${ }^{3}$. V. K. Kalevich . $^{3}$

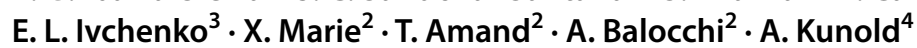

Received: 31 October 2017 / Accepted: 20 February 2018

(c) Springer Science+Business Media, LLC, part of Springer Nature 2018

\begin{abstract}
The impact of $\mathrm{Ga}^{3+}$ centers in the spin-filtering effect observed in GaAsN samples is investigated through a model based on the master equation approach. Our results, compared with experimental data, show that, $\mathrm{Ga}^{3+}$ are essential to understanding the behavior of the photoluminescence intensity and degree of circular polarization as functions of a Faraday configuration magnetic field. The model presented here takes into account the interplay of $\mathrm{Ga}^{2+}$ and $\mathrm{Ga}^{3+}$ centers, Zeeman and hyperfine interaction. The various processes that drive the spin-filtering effect, as the spin selective capture of conduction band electrons into Ga centers are also considered here.
\end{abstract}

\section{Introduction}

GaAsN dilute semiconductors have attracted significant attention mainly due to their applications in telecommunications [1], photovoltaic devices with efficiency close to $50 \%$ [2], and optoelectronic devices in general [3]. The unusually large bowing parameter of semiconductor alloys containing $\mathrm{N}$ considerably diminishes their band gap $[4,5]$ allowing to tune their emission wave length within the telecommunication range [6]. Even though the incorporation of a small amount of $\mathrm{N}$ in the crystal lattice of GaAs deteriorates the photoluminescence and carrier lifetime [7, 8], it introduces new and interesting properties regarding the spin polarization of conduction band (CB) electrons [6]. The longer spin relaxation times and record spin polarizations (almost 100\%) attained at room temperature under circularly polarized light

V. G. Ibarra-Sierra

vibarra@xanum.uam.mx

1 Departamento de Física, Universidad Autónoma Metropolitana, Iztapalapa, Av. San Rafael Atlixco 186, Col. Vicentina, 09340 Mexico City, Mexico

2 Universitè de Toulouse, INSA-CNRS-UPS, LPCNO, 135 avenue de Rangueil, 31077 Toulouse, France

3 Ioffe Physical-Technical Institute, St. Petersburg 194021, Russia

4 Área de Física Teórica y Materia Condensada, Universidad Autónoma Metropolitana Azcapotzalco, Av. San Pablo 180, Col. Reynosa-Tamaulipas, 02200 Mexico City, Mexico excitation are mainly due to Ga spin-filtering defects [9-17]. This phenomenon, commonly termed as a defect-engineered spin-filtering effect, relies on the selective capture of oneelectron according to its spin orientation [15, 16, 18-20]. Later experiments on GaAsN subject to a weak magnetic field in Faraday configuration [21-25] revealed that the hyperfine interaction (HFI), which couples the electronic and nuclear spins in Ga centers, plays a key role in the spinfiltering mechanism. The HFI mixes the bound electron spin states mainly producing three effects [25-27]: (i) a reduction of the spin polarization in zero magnetic field, (ii) a dynamical polarization of the nuclear spins and (iii) an Overhauserlike magnetic field. Most of the observed results can be qualitatively and, in some cases, quantitatively reproduced by a generalization of the well known two charge model [9, 10, 15, 19, 28].

Even though presently the general agreement is that $\mathrm{Ga}^{2+}$ are the spin-filtering centers, there is a great amount of experimental and theoretical evidence pointing to the existence of other types of $\mathrm{Ga}$ interstitial defects (e.g. $\mathrm{Ga}^{3+}$ ) [29-32] that could, in principle, intervene in the spin-filtering effect. Early research on dopant diffusion in GaAs suggests that divalent elements such as Zn [29, 30] and Cd [31] mostly substitute into the Ga sublattice producing Ga interstitial defects through the kick-out mechanism. Using secondary ion-mass spectroscopy on ion-implanted $\mathrm{Zn}$ diffusion, it was found that a quadratic diffusion law characterizes the $\mathrm{Zn}$ diffusion implying thermal equilibrium and doubly or triply charged $\mathrm{Ga}$ interstitial defects [29, 30]. Similar studies with ion-implanted Cd diffusion [31] 


\title{
Method for Finding the Exact Effective Hamiltonian of Time-Driven Quantum Systems
}

\author{
Juan Carlos Sandoval-Santana, Victor Guadalupe Ibarra-Sierra, José Luis Cardoso, \\ Alejandro Kunold, * Pedro Roman-Taboada, and Gerardo García Naúmis
}

Time-driven quantum systems are important in many different fields of physics as cold atoms, solid state, optics, etc. Many of their properties are encoded in the time evolution operator or the effective Hamiltonian. Finding these operators usually requires very complex calculations that often involve some approximations. To perform this task, a systematic scheme that can be cast in the form of a symbolic computational algorithm is presented. It is suitable for periodic and non-periodic potentials and, for convoluted systems, can also be adapted to yield numerical solutions. The method exploits the structure of the associated Lie group and a decomposition of the evolution operator on each group generator. To illustrate the use of the method, five examples are provided: harmonic oscillator with time-dependent frequency (Paul trap), modulated optical lattice, time-driven quantum oscillator, a step-wise driving of a free particle, and the non-periodic Caldirola-Kanai Hamiltonian. To the extent of the authors' knowledge, whereas the exact form of Paul trap's evolution operator is well known, its effective Hamiltonian was until now unknown. The remaining four examples accurately reproduce previous results.
$H(t)=H_{0}+V(t)$ typically consist of a time-independent Hamiltonian $\mathrm{H}_{0}$ plus a time-dependent potential $V(t)$. In this paper, particular attention will be paid to the case where $V(t)$ is periodic although the results may be applied to non-periodical potentials. Notice that for periodic driving, there is an approximated method, ${ }^{[19]}$ based in the Wegner's flow method, ${ }^{[20]}$ which solves the problem up to a required accuracy, yet it requires periodicity.

The TDQS properties are thus calculated by using the time evolution operator $U(t)=\mathscr{T} \exp \left[-i \int_{0}^{t} d t H(t) / \hbar\right]$, where $\mathscr{T}$ is the time ordering operator. In the case of periodic potentials, using Floquet theory, ${ }^{[21,22]}$ one can show that the solution is equivalent to finding an effective Hamiltonian $H_{e}$ such that ${ }^{[5]}$

$$
U(T)=\mathrm{e}^{-i H_{\mathrm{e}} T / \hbar}
$$

\section{Introduction}

In recent years, there has been a remarkable interest in time-driven quantum systems ${ }^{[1-5]}$ (TDQS). Among the reasons for this are the possibility of tailoring time-driven potentials using cold atoms, ${ }^{[6-11]}$ creating topologically protected edge states, ${ }^{[12,13]}$ other interesting topological phases, ${ }^{[14-16]}$ and dynamically generating quantum entanglement ${ }^{[17]}$ as well as global quantum-quenching. ${ }^{[18]}$ The Hamiltonians of these systems ${ }^{[5}$

\footnotetext{
Dr. J. C. Sandoval-Santana, ${ }^{[+]}$Prof. J. L. Cardoso, Prof. A. Kunold Departamento de Ciencias Básicas

Universidad Autónoma Metropolitana Azcapotzalco

Av. San Pablo 180, Col. Reynosa Tamaulipas, 02200 Ciudad de México, México

E-mail: akb@azc.uam.mx

Dr. V. G. Ibarra-Sierra, Dr. P. Roman-Taboada, Prof. G. G. Naúmis

Departamento de Sistemas Complejos, Instituto de Física

Universidad Nacional Autónoma de México

Apartado Postal 20-364, 01000 Ciudad de México, México
}

${ }^{[+]}$Present address: Instituto de Física, Universidad Nacional Autónoma de México, Apartado Postal 20-364, 01000 Ciudad de México, México

The ORCID identification number(s) for the author(s) of this article can be found under https://doi.org/10.1002/andp.201900035

DOI: 10.1002/andp.201900035 where $T$ is the period of the potential, plus the evolution from the end of the last cycle to $t$, this last part is related to the micromotion. ${ }^{[5]}$ The calculation of $H_{e}$ is not a trivial task and only few cases allow a closed analytic solution..$^{[5]}$

Here, we present a method based on the use of Lie algebras to compute $H_{e} \cdot{ }^{[23]}$ A great variety of physically relevant Hamiltonians may be addressed by this method. Examples are: the modulated optical lattice, ${ }^{[9,24]}$ fastly driven tight-binding chains, ${ }^{[25,26]}$ Paul trap, ${ }^{[27]}$ quantum wires, ${ }^{[28]}$ graphene, ${ }^{[29]}$ quantum parametric resonance, ${ }^{[30]}$ and Hubbard Hamiltonian. ${ }^{[8,31,32]}$ The method can also be used to find gauge transformations that eliminate the time dependence from certain Hamiltonians..$^{[5,33]}$ This approach is based on our earlier results on the use of Lie algebras to find the evolution operator of time-dependent Hamitlonians. ${ }^{[34,35]}$ However, the method presented here has major improvements and differences with respect to our previous work. First, it does not require the prior knowledge of the transformation rules arising from the commutators between the algebra's elements. Moreover, the transformation rules are obtained directly from the structure constants as part of the method. Second, it enables us to put the evolution operator in the form of a single unitary transformation, that, as we will see later (see Equation (5)), is a crucial step in finding the effective Hamiltonian. In contrast, our earlier results only allow to compute the evolution operator in the form of a product of unitary transformations (see Equation (4)). For convoluted systems that do not allow an analytic solution, the 


\section{Bibliografía}

[1] V. G. Ibarra-Sierra, J. C. Sandoval-Santana, S. Azaizia, H. Carrère, L. A. Bakaleinikov, V. K. Kalevich, E. L. Ivchenko, X. Marie, T. Amand, A. Balocchi, and A. Kunold. Electron-nuclear spin dynamics of $\mathrm{Ga}^{2+}$ paramagnetic centers probed by spin-dependent recombination: A master equation approach. Phys. Rev. B, 95:195204, May 2017.

[2] S. Azaizia, H. Carrère, J. C. Sandoval-Santana, V. G. Ibarra-Sierra, V. K. Kalevich, E. L. Ivchenko, L. A. Bakaleinikov, X. Marie, T. Amand, A. Kunold, and A. Balocchi. Electron-nuclear coherent spin oscillations probed by spin-dependent recombination. Phys. Rev. B, 97:155201, Apr 2018 .

[3] JC Sandoval-Santana, VG Ibarra-Sierra, S Azaizia, H Carrère, LA Bakaleinikov, VK Kalevich, EL Ivchenko, X Marie, T Amand, A Balocchi, et al. Electron-nuclear spin dynamics of ga centers in GaAsN dilute nitride semiconductors probed by pump-probe spectroscopy. The European Physical Journal Plus, 133(3):122, 2018.

[4] V. Kalevich, E. Ivchenko, M. Afanasiev, A. Shiryaev, A. Egorov, V. Ustinov, B. Pal, and Y. Masumoto. Spin-dependent recombination in GaAsN solid solutions. JETP Letters, 82:455-458, 2005.

[5] V. Kalevich, A. Shiryaev, E. Ivchenko, A. Egorov, L. Lombez, D. Lagarde, X. Marie, and T. Amand. Spin-dependent electron dynamics and recombination in GaAs alloys at room temperature. JETP Letters, 85:174-178, 2007.

[6] Georges Lampel. Nuclear dynamic polarization by optical electronic saturation and optical pumping in semiconductors. Phys. Rev. Lett., 20:491-493, Mar 1968.

[7] P. C. Maurer, G. Kucsko, C. Latta, L. Jiang, N. Y. Yao, S. D. Bennett, F. Pastawski, D. Hunger, N. Chisholm, M. Markham, D. J. Twitchen, J. I. Cirac, and M. D. Lukin. Room-temperature quantum bit memory exceeding one second. Science, 336(6086):1283-1286, 2012. 
[8] Sang-Yun Lee, Matthias Widmann, Torsten Rendler, Marcus W Doherty, Thomas M Babinec, Sen Yang, Moritz Eyer, Petr Siyushev, Birgit JM Hausmann, Marko Loncar, et al. Readout and control of a single nuclear spin with a metastable electron spin ancilla. Nature nanotechnology, 8(7):487-492, 2013.

[9] Adrian Auer and Guido Burkard. Phys. Rev. B, 93:035402, Jan 2016.

[10] T. van der Sar, Z. H. Wang, M. S. Blok, H. Bernien, T. H. Taminiau, D. M. Toyli, D. A. Lidar, D. D. Awschalom, R. Hanson, and V. V. Dobrovitski. Nature, 484(7392):82-86, 042012.

[11] N. Mizuochi, P. Neumann, F. Rempp, J. Beck, V. Jacques, P. Siyushev, K. Nakamura, D. J. Twitchen, H. Watanabe, S. Yamasaki, F. Jelezko, and J. Wrachtrup. Phys. Rev. B, 80:041201, Jul 2009.

[12] J. R. Maze, J. M. Taylor, and M. D. Lukin. Phys. Rev. B, 78:094303, Sep 2008.

[13] Jarryd J. Pla, Fahd A. Mohiyaddin, Kuan Y. Tan, Juan P. Dehollain, Rajib Rahman, Gerhard Klimeck, David N. Jamieson, Andrew S. Dzurak, and Andrea Morello. Phys. Rev. Lett., 113:246801, Dec 2014.

[14] R. Hanson, V. V. Dobrovitski, A. E. Feiguin, O. Gywat, and D. D. Awschalom. Science, 320(5874):352-355, 2008.

[15] B. E. Kane. A silicon-based nuclear spin quantum computer. Nature, 393(6681):133-137, May 1998.

[16] Jarryd J. Pla, Kuan Y. Tan, Juan P. Dehollain, Wee H. Lim, John J. L. Morton, David N. Jamieson, Andrew S. Dzurak, and Andrea Morello. Nature, 489(7417):541-545, 092012.

[17] T. D. Ladd, D. Maryenko, Y. Yamamoto, E. Abe, and K. M. Itoh. Phys. Rev. B, 71:014401, Jan 2005.

[18] Arne Laucht, Rachpon Kalra, Juha T. Muhonen, Juan P. Dehollain, Fahd A. Mohiyaddin, Fay Hudson, Jeffrey C. McCallum, David N. Jamieson, Andrew S. Dzurak, and Andrea Morello. High-fidelity adiabatic inversion of a $31 \mathrm{p}$ electron spin qubit in natural silicon. Appl. Phys. Lett., 104(9), 2014.

[19] Wang Yao, Ren-Bao Liu, and L. J. Sham. Phys. Rev. B, 74:195301, Nov 2006. 
[20] Viktor Ivády, Krisztián Szász, Abram L. Falk, Paul V. Klimov, David J. Christle, Erik Janzén, Igor A. Abrikosov, David D. Awschalom, and Adam Gali. Phys. Rev. B, 92:115206, Sep 2015.

[21] D. Simin, V. A. Soltamov, A. V. Poshakinskiy, A. N. Anisimov, R. A. Babunts, D. O. Tolmachev, E. N. Mokhov, M. Trupke, S. A. Tarasenko, A. Sperlich, P. G. Baranov, V. Dyakonov, and G. V. Astakhov. Alloptical dc nanotesla magnetometry using silicon vacancy fine structure in isotopically purified silicon carbide. Phys. Rev. X, 6:031014, Jul 2016.

[22] Takayuki Iwasaki, Fumitaka Ishibashi, Yoshiyuki Miyamoto, Yuki Doi, Satoshi Kobayashi, Takehide Miyazaki, Kosuke Tahara, Kay D Jahnke, Lachlan J Rogers, Boris Naydenov, et al. Germanium-vacancy single color centers in diamond. Scientific reports, 5, 2015.

[23] Yuri N Palyanov, Igor N Kupriyanov, Yuri M Borzdov, and Nikolay V Surovtsev. Germanium: a new catalyst for diamond synthesis and a new optically active impurity in diamond. Scientific reports, 5, 2015.

[24] Petr Siyushev, Mathias H. Metsch, Aroosa Ijaz, Jan M. Binder, Mihir K. Bhaskar, Denis D. Sukachev, Alp Sipahigil, Ruffin E. Evans, Christian T. Nguyen, Mikhail D. Lukin, Philip R. Hemmer, Yuri N. Palyanov, Igor N. Kupriyanov, Yuri M. Borzdov, Lachlan J. Rogers, and Fedor Jelezko. Optical and microwave control of germanium-vacancy center spins in diamond. Phys. Rev. B, 96:081201, Aug 2017.

[25] A. Gruber, A. Dräbenstedt, C. Tietz, L. Fleury, J. Wrachtrup, and C. von Borczyskowski. Scanning confocal optical microscopy and magnetic resonance on single defect centers. Science, 276(5321):2012-2014, 1997.

[26] Kannan Ramaswamy, Stacy Mui, and Sophia E. Hayes. Light-induced hyperfine ${ }^{69} \mathrm{Ga}$ shifts in semi-insulating GaAs observed by optically polarized nmr. Phys. Rev. B, 74:153201, Oct 2006.

[27] Anne S. Verhulst, Ileana G. Rau, Yoshihisa Yamamoto, and Kohei M. Itoh. Optical pumping of ${ }^{29} \mathrm{Si}$ nuclear spins in bulk silicon at high magnetic field and liquid helium temperature. Phys. Rev. B, 71:235206, Jun 2005 .

[28] Erika L. Sesti, Matthew M. Willmering, Zayd L. Ma, Dustin D. Wheeler, Mark S. Conradi, and Sophia E. Hayes. A combined experimental setup for op and odnmr. Journal of Magnetic Resonance, 281(Supplement C):172 - 187, 2017. 
[29] Y. Puttisong, D. Dagnelund, I. A. Buyanova, C. W. Tu, A. Polimeni, M. Capizzi, and W. M. Chen. Room temperature spin filtering effect in ganas: Role of hydrogen. Applied Physics Letters, 99(15):152109, 2011.

[30] X. J. Wang, Y. Puttisong, C. W. Tu, Aaron J. Ptak, V. K. Kalevich, A. Yu. Egorov, L. Geelhaar, H. Riechert, W. M. Chen, and I. A. Buyanova. Dominant recombination centers in ga(in)nas alloys: Ga interstitials. Appl. Phys. Lett., 95(24):241904, 2009.

[31] Jonathan P King, Keunhong Jeong, Christophoros C Vassiliou, Chang S Shin, Ralph H Page, Claudia E Avalos, Hai-Jing Wang, and Alexander Pines. Room-temperature in situ nuclear spin hyperpolarization from optically pumped nitrogen vacancy centres in diamond. Nature communications, 6:8965, 2015.

[32] S. A. Crooker, D. D. Awschalom, J. J. Baumberg, F. Flack, and N. Samarth. Optical spin resonance and transverse spin relaxation in magnetic semiconductor quantum wells. Phys. Rev. B, 56:7574-7588, Sep 1997.

[33] P. Liang, R. R. Hu, C. Chen, V. V. Belykh, T. Q. Jia, Z. R. Sun, D. H. Feng, D. R. Yakovlev, and M. Bayer. Room-temperature electron spin dynamics of Ce3+ ions in a yag crystal. Appl. Phys. Lett., 110(22):222405, 2017.

[34] D. V. Azamat, V. V. Belykh, D. R. Yakovlev, F. Fobbe, D. H. Feng, E. Evers, L. Jastrabik, A. Dejneka, and M. Bayer. Electron spin dynamics of $\mathrm{ce}^{3+}$ ions in yag crystals studied by pulse-epr and pump-probe faraday rotation. Phys. Rev. B, 96:075160, Aug 2017.

[35] Hiroshi Watanabe, Takayuki Kurihara, Takeo Kato, Keita Yamaguchi, and Tohru Suemoto. Observation of long-lived coherent spin precession in orthoferrite erfeo3 induced by terahertz magnetic fields. Applied Physics Letters, 111(9):092401, 2017.

[36] D. H. Feng, L. F. Shan, T. Q. Jia, X. Q. Pan, H. F. Tong, L. Deng, Z. R. Sun, and Z. Z. Xu. Optical manipulation of electron spin coherence in colloidal CdS quantum dots. Applied Physics Letters, 102(6):062408, 2013.

[37] AV Kimel, A Kirilyuk, A Tsvetkov, RV Pisarev, and Th Rasing. Laserinduced ultrafast spin reorientation in the antiferromagnet tmfeo3. $\mathrm{Na}$ ture, 429(6994):850, 2004. 
[38] Sebastian Loth, Markus Etzkorn, Christopher P. Lutz, D. M. Eigler, and Andreas J. Heinrich. Measurement of fast electron spin relaxation times with atomic resolution. Science, 329(5999):1628-1630, 2010.

[39] X.J. Wang, I.A. Buyanova, F. Zhao, D. Lagarde, A. Balocchi, X. Marie, C.W. Tu, J.C. Harmand, and W.M. Chen. Room-temperature defectengineered spin filter based on a non-magnetic semiconductor. Nat $\mathrm{Ma}$ ter, 8(3):198-202, 2009.

[40] Yuttapoom Puttisong, XJ Wang, IA Buyanova, L Geelhaar, H Riechert, AJ Ptak, CW Tu, and WM Chen. Efficient room-temperature nuclear spin hyperpolarization of a defect atom in a semiconductor. Nature communications, 4:1751, 2013.

[41] L. Lombez, P.-F. Braun, H. Carrère, B. Urbaszek, P. Renucci, T. Amand, X. Marie, J.C. Harmand, and V.K. Kalevich. Spin dynamics in dilute nitride semiconductors at room temperature. Applied Physics Letters, 87(25):252115, 2005.

[42] F. Zhao, A. Balocchi, A. Kunold, J. Carrey, H. Carrere, T. Amand, N. Ben Abdallah, J. C. Harmand, and X. Marie. Spin-dependent photoconductivity in nonmagnetic semiconductors at room temperature. Applied Physics Letters, 95(24):241104, 2009.

[43] C. Sandoval-Santana, A. Balocchi, T. Amand, J. C. Harmand, A. Kunold, and X. Marie. Room-temperature optical manipulation of nuclear spin polarization in GaAsN. Phys. Rev. B, 90:115205, Sep 2014.

[44] B. E. Kane. Nature, 393(6681):133-137, May 1998.

[45] Arne Laucht, Rachpon Kalra, Juha T. Muhonen, Juan P. Dehollain, Fahd A. Mohiyaddin, Fay Hudson, Jeffrey C. McCallum, David N. Jamieson, Andrew S. Dzurak, and Andrea Morello. Appl. Phys. Lett., 104(9), 2014.

[46] D. D. Bhaktavatsala Rao, Sen Yang, and Jörg Wrachtrup. Phys. Rev. B, 92:081301, Aug 2015.

[47] Sebastian Zaiser, Torsten Rendler, Ingmar Jakobi, Thomas Wolf, SangYun Lee, Samuel Wagner, and Bergholm. Nat. Comm., 7:12279, August 2016 .

[48] Y. Doi, T. Makino, H. Kato, D. Takeuchi, M. Ogura, H. Okushi, H. Morishita, T. Tashima, S. Miwa, S. Yamasaki, P. Neumann, J. Wrachtrup, 
Y. Suzuki, and N. Mizuochi. Deterministic electrical charge-state initialization of single nitrogen-vacancy center in diamond. Phys. Rev. X, 4:011057, Mar 2014.

[49] M. W. Doherty, C. A. Meriles, A. Alkauskas, H. Fedder, M. J. Sellars, and N. B. Manson. Towards a room-temperature spin quantum bus in diamond via electron photoionization, transport, and capture. Phys. Rev. X, 6:041035, Nov 2016.

[50] Kae Nemoto, Michael Trupke, Simon J. Devitt, Ashley M. Stephens, Burkhard Scharfenberger, Kathrin Buczak, Tobias Nöbauer, Mark S. Everitt, Jörg Schmiedmayer, and William J. Munro. Photonic architecture for scalable quantum information processing in diamond. Phys. Rev. X, 4:031022, Aug 2014.

[51] A. Kunold, A. Balocchi, F. Zhao, T. Amand, N. Ben Abdallah, J.C. Harmand, and X. Marie. Giant spin-dependent photo-conductivity in GaAsN dilute nitride semiconductor. Phys. Rev. B, 83:165202, 2011.

[52] E. L. Ivchenko, L. A. Bakaleinikov, and V. K. Kalevich. Spin-dependent recombination and hyperfine interaction at deep defects. Phys. Rev. B, 91:205202, May 2015.

[53] Rodrigo Gutiérrez-Cuevas and Joseph H Eberly. Manipulation of optical-pulse-imprinted memory in a $\lambda$ system. Physical Review A, 92(3):033804, 2015.

[54] JC Sandoval-Santana, VG Ibarra-Sierra, JL Cardoso, and A Kunold. Time evolution of two-dimensional quadratic hamiltonians: A lie algebraic approach. Journal of Mathematical Physics, 57(4):042104, 2016.

[55] D. Lagarde, L. Lombez, X. Marie, A. Balocchi, T. Amand, V.K. Kalevich, A. Shiryaev, E. Ivchenko, and A. Egorov. Electron spin dynamics in GaAsN and InGaAsN structures. physica status solidi (a), 204(1):208220, 2007.

[56] S. Wei and A. Zunger. Giant and composition-dependent optical bowing coefficient in GaAsN alloys. Phys. Rev. Lett., 76:664-667, 1996.

[57] E.D. Jones, N.A. Modine, A.A. Allerman, S.R. Kurtz, A.F. Wright, S.T. Tozer, and X. Wei. Band structure of $\operatorname{In}_{x} \mathrm{Ga}_{1-x} \mathrm{As}_{1-y} \mathrm{~N}_{y}$ alloys and effects of pressure. Phys. Rev. B, 60:4430-4433, 1999. 
[58] W. Shan, W. Walukiewicz, J.W. Ager, E.E. Haller, J.F. Geisz, D.J. Friedman, J.M. Olson, and S.R. Kurtz. Band anticrossing in gainnas alloys. Phys. Rev. Lett., 82:1221-1224, 1999.

[59] H.Ch Alt, A Egorov, H. Riechert, B. Wiedemann, J.D. Meyer, R.W. Michelmann, and K. Bethge. Local vibrational mode absorption of nitrogen in GaAsN and InGaAsN layers grown by molecular beam epitaxy. Physica B: Condensed Matter, 302-303:282 - 290, 2001.

[60] C. Cohen-Tannoudji, B. Diu, and F. Laloë. Quantum mechanics. Quantum Mechanics. Wiley, 1977.

[61] D.J. Lepine. Spin-dependent recombination on silicon surface. Phys. Rev. B, 6:436-441, 1972.

[62] T. Wosinski, T. Figielski, and A. Makosa. Spin-dependent photoconductivity spectrum of dislocated silicon. physica status solidi (a), 37(1):K57K59, 1976.

[63] R.M. White and J.F. Gouyet. Theory of spin-dependent effects in silicon. Phys. Rev. B, 16:3596-3602, 1977.

[64] I. Solomon, D. Biegelsen, and J.C. Knights. Spin-dependent photoconductivity in n-type and p-type amorphous silicon. Solid State Communications, 22(8):505-508, 1977.

[65] D. Paget. Optical-pumping study of spin-dependent recombination in GaAs. Phys. Rev. B, 30:931-946, 1984.

[66] G. Slavcheva and P. Roussignol. Optical Generation and Control of Quantum Coherence in Semiconductor Nanostructures. NanoScience and Technology. Springer Berlin Heidelberg, 2010.

[67] MV Gurudev Dutt, L Childress, L Jiang, E Togan, J Maze, F Jelezko, AS Zibrov, PR Hemmer, and MD Lukin. Quantum register based on individual electronic and nuclear spin qubits in diamond. Science, 316(5829):1312-1316, 2007.

[68] H.P. Breuer, F. Petruccione, P.I.H.P. Breuer, and S.P.A.P.F. Petruccione. The Theory of Open Quantum Systems. Oxford University Press, 2002 .

[69] Rodrigo Gutiérrez-Cuevas and Joseph H. Eberly. Manipulation of optical-pulse-imprinted memory in a $\Lambda$ system. Phys. Rev. A, 92:033804, Sep 2015. 
[70] C. Weisbuch and G. Lampel. Spin-dependent recombination and optical spin orientation in semiconductors. Solid State Communications, 14(2):141 - 144, 1974.

[71] D.J. Hilton and C.L. Tang. Optical orientation and femtosecond relaxation of spin-polarized holes in GaAs. Phys. Rev. Lett., 89:146601, 2002.

[72] C.D. Cantrell. Modern Mathematical Methods for Physicists and Engineers. Cambridge University Press, 2000.

[73] HM Zhao, L Lombez, BL Liu, BQ Sun, QK Xue, DM Chen, and X Marie. Electron spin quantum beats and room temperature g factor in GaAsN. Applied Physics Letters, 95(4):041911, 2009.

[74] V.K. Kalevich, M.M. Afanasiev, A.Yu. Shiryaev, and A.Yu. Egorov. Amplification of spin-filtering effect by magnetic field in GaAsN alloys. Phys. Rev. B, 85:035205, 2012.

[75] H.P. Breuer and F. Petruccione. The Theory of Open Quantum Systems. Oxford University Press, 2002.

[76] E.L. Ivchenko, V.K. Kalevich, A. Yu Shiryaev, M.M. Afanasiev, and Y. Masumoto. Optical orientation and spin-dependent recombination in GaAsN alloys under continuous-wave pumping. Journal of Physics: Condensed Matter, 22(46):465804, 2010.

[77] V.K. Kalevich, A.Yu. Shiryaev, E.L. Ivchenko, M.M. Afanasiev, A.Yu. Egorov, V.M. Ustinov, and Y. Masumoto. Hanle effect and spindependent recombination at deep centers in GaAsN. Physica B: Condensed Matter, 404(23“24):4929 - 4932, 2009.

[78] R. K. Wangsness and F. Bloch. Phys. Rev., 89:728-739, Feb 1953.

[79] A.G. REDFIELD. In John S. Waugh, editor, Advances in Magnetic Resonance, volume 1 of Advances in Magnetic and Optical Resonance, pages 1 - 32. Academic Press, 1965.

[80] G. W. Leppelmeier and E. L. Hahn. Phys. Rev., 142:179-189, Feb 1966.

[81] J. Kowalewski and L. Maler. Series in Chemical Physics. CRC Press, 2006.

[82] Inc. Wolfram Research. Mathematica 11.0.1.0. Champaign, IL 618207237, USA, 1988-2015. 
[83] X. J. Wang, Y. Puttisong, C. W. Tu, Aaron J. Ptak, V. K. Kalevich, A. Yu. Egorov, L. Geelhaar, H. Riechert, W. M. Chen, and I. A. Buyanova. Appl. Phys. Lett., 95(24), 2009.

[84] VG Ibarra-Sierra, JC Sandoval-Santana, JL Cardoso, and A Kunold. Lie algebraic approach to the time-dependent quantum general harmonic oscillator and the bi-dimensional charged particle in time-dependent electromagnetic fields. Annals of Physics, 362:83-117, 2015.

[85] D Velasco-Martínez, VG Ibarra-Sierra, JC Sandoval-Santana, JL Cardoso, and A Kunold. Singularities and internal rotational dynamics of electron beams. Physical Review A, 94(6):063815, 2016.

[86] VG Ibarra-Sierra, JC Sandoval-Santana, S Azaizia, H Carrère, LA Bakaleinikov, VK Kalevich, EL Ivchenko, X Marie, T Amand, A Balocchi, et al. Spin-filtering effect in gaasn: electron-nuclear spin dynamics of ga 3+ centers. Journal of Materials Science: Materials in Electronics, pages 1-8, 2018.

[87] Juan Carlos Sandoval-Santana, Victor Guadalupe Ibarra-Sierra, José Luis Cardoso, Alejandro Kunold, Pedro Roman-Taboada, and Gerardo García Naumis. Method for finding the exact effective hamiltonian of time-driven quantum systems. Annalen der Physik, 0(0):1900035. 